\title{
Optimal Financial Transaction Taxes
}

\author{
Eduardo Dávila* \\ Harvard University
}

Job MARKET PAPER

November 2013

\begin{abstract}
This paper characterizes the optimal linear financial transaction tax in an equilibrium model of competitive financial markets. When belief disagreement induces excess trading on assets in fixed supply, two main results arise. First, the optimal tax is positive: although a (small) transaction tax discourages all trades equally, the reduction in fundamental trading creates a second-order welfare loss, while the reduction in non-fundamental trading creates a first-order gain. Second, the crosssectional covariance between investors' beliefs and investors' equilibrium portfolio tax sensitivities becomes the relevant sufficient statistic for the optimal tax, which does not depend on the actual payoff distribution. I find additional results. First, in dynamic environments, controlling for the level of static disagreement, the optimal tax is lower when investors alternate between being buyers and sellers over time. Second, when financial markets determine production in a Walrasian sense, as in a q-theory environment, a marginal tax increase creates an additional first-order distortion (positive or negative). Third, when financial markets determine production by diffusing information, a marginal tax increase creates an additional first-order loss, due to a learning externality.
\end{abstract}

JEL Classification: G18, H21, G02.

Keywords: financial transaction tax, Tobin tax, transaction costs, belief disagreement, heterogeneous priors, noise trading, optimal taxation, sufficient statistics

\footnotetext{
*Contact: edavila@fas.harvard.edu. Check http://scholar.harvard.edu/edavila for the most updated version. I would like to especially thank John Campbell, Emmanuel Farhi, Jeremy Stein and Alp Simsek for many helpful comments and advice. I am very grateful to Philippe Aghion, Robert Barro, Markus Brunnermeier, Raj Chetty, Chris Edmond, Jordi Galí, Gita Gopinath, Robin Greenwood, Sam Hanson, Oliver Hart, Nathan Hendren, David Laibson, Greg Mankiw, Sendhil Mullainathan, Jonathan Parker, Adriano Rampini, David Scharfstein, Andrei Shleifer, Adi Sunderam and Luis Viceira for helpful comments. I also thank participants in Harvard Macro lunch, Organizations and Contracts lunch, Public Finance seminar and Harvard/HBS Finance lunch. Financial support from Rafael del Pino Foundation is gratefully acknowledged.
} 


\section{Introduction}

Motivation Should we set a financial transaction tax to curb speculation generated by distorted beliefs? Wouldn't a "Tobin" tax also prevent welfare enhancing trades from being executed? These questions periodically arise after periods of financial instability. For instance, Tobin's well-known 1972 speech follows the collapse of the Bretton Woods system, Stiglitz (1989) and Summers and Summers (1989) write on the subject after the 1987 crash and, spurred by the 2008 financial crisis, the European Commission seems eager to impose a transaction tax. However, as recently posed by Cochrane (2013), a financial transaction tax may seem "the perennial favorite answer in search of a question". This paper provides a framework to understand the welfare implications of taxing financial transactions.

Financial markets play three distinct roles. First, they allow investors to conduct fundamental trading. Fundamental trading allows for risk sharing among similar investors or risk transfer to those investors who can better bear risk. It also allows for liquidity or life-cycle trading needs, as well as trading for market-making or (limited) arbitrage purposes. Second, financial markets allow investors to engage in betting or gambling - I refer to this as non-fundamental trading. In this paper, I capture this notion by assuming that investors hold heterogeneous beliefs. Third, financial markets determine production and investment in two different ways. On the one hand, in a Walrasian sense, they determine the relative scarcity of a given asset/technology, enticing investors to supply more or less of it. On the other hand, in a Hayekian sense, financial markets facilitate the diffusion of information from investors to managers, improving production efficiency. This paper analyzes how all three roles determine the sign and the magnitude of the optimal tax.

Main results I initially lay out a static CARA-Normal model of an exchange economy to understand the tradeoff between fundamental and non-fundamental trading.

First, I show that when some investors hold distorted beliefs about expected payoffs it is always optimal to set a corrective tax, which, in theory, can be positive or negative (a subsidy). When belief disagreement induces investors to trade too much, the optimal financial tax is strictly positive. Even though introducing a (small) ${ }^{1}$ transaction tax distorts investors' portfolio allocations towards their notrade positions, the reduction in fundamental trading creates a second-order welfare loss while the reduction in non-fundamental trading creates a first-order gain. Intuitively, because fundamental trades are done optimally, the envelope theorem guarantees that the (local) welfare loss derived from reducing them is second-order. However, preventing non-fundamental trades always creates a first-order welfare gain, because these trades are done suboptimally from the planner's perspective.

Second, I characterize the optimal transaction tax. The optimal tax balances globally the welfare gains generated by reducing non-fundamental trades with the welfare losses generated by distorting fundamental trades. A potentially observable object, the cross-sectional covariance between investors' beliefs and equilibrium portfolio tax sensitivities becomes the single relevant sufficient statistic for the optimal tax. The actual distribution of payoffs turns out to be irrelevant to determine the optimal tax;

\footnotetext{
${ }^{1}$ By small tax, I mean tax changes in the neighborhood of $\tau=0$.
} 
this striking result relies on two assumptions: a) traded assets are in fixed supply and b) the planner does not seek to redistribute income across investors.

Additional results Within the static framework, I explore several extensions. First, the optimal tax formula remains unchanged when there are pre-existing trading costs, as long as these are compensation for the use of economic resources, not economic rents. Second, in an environment with multiple risky assets, the optimal tax becomes a weighted average of the optimal taxes for each individual asset. Third, the optimal tax formula remains unchanged in the presence of price independent portfolio constraints, such as short-sale constraints. It has to be modified to account for price dependent borrowing constraints. Fourth, the optimal tax formula can be adapted to incorporate distortions in second moments. Fifth, I show that the planner would need investor-specific taxes in order to implement the first-best outcome.

Extending the simple static model to a dynamic environment with general utility functions and an arbitrary form of disagreement generates three new insights. First, an approximation of the optimal tax formula around the point at which investors are approximately risk neutral recovers the optimal tax from the simple CARA-Normal model. Second, when investors alternate between buying and selling, the optimal tax in a dynamic environment is smaller in magnitude, controlling for the level of static disagreement. Intuitively, a transaction tax is more effective with forward-looking investors who expect to alternate between buying and selling over time, since the anticipation of future taxes further reduces the incentives to trade today. Third, I show that, even for a planner who maximizes welfare respecting the beliefs of each individual investor, there may exist a rationale for taxation when markets are incomplete. This result shows that price volatility - an often used measure in policy discussionsis not the correct criterion to determine the welfare effects of a transaction tax. I show that, in this case, the time-series covariance between price sensitivities with respect to a tax change and marginal utility weighted changes in net trading positions becomes the welfare relevant sufficient statistic price volatility (a variance) has no direct correspondence with welfare.

Lastly, I extend the results to production economies. First, in a Walrasian production economy, a (small) transaction tax creates an additional first-order gain or loss as long as investors' beliefs are on average incorrect. In addition to the correct allocation of risk among investors, it now matters whether the level of aggregate risk in the economy or its allocation across sectors is also correct. Second, in an economy in which managers learn information useful for production through financial markets, a (small) transaction tax creates an additional first-order loss because, by making prices less informative, it reduces production efficiency.

Welfare criterion For most of the results, the planner in this paper calculates welfare using a single distribution of payoffs. Although the planner does not respect investors' beliefs, I characterize the circumstances under which the optimal policy is independent of the belief chosen by the planner. In those cases, the planner does not enjoy any informational advantage over the investors. In dynamic environments and in production economies, the belief used by the planner matters in general: I precisely 
characterize how it affects optimal policies.

However, two results in this paper assume that the planner respects investors' beliefs. First, in a static setup, I derive an expression that quantifies the welfare loss induced by a marginal tax change when all trades are regarded as fundamental — this is equivalent to the Harberger (1964) triangle calculation. This expression provides an upper bound for losses in social welfare, since it assumes that there are no welfare gains from taxation. Second, in a dynamic setup with incomplete markets, I show that a tax/subsidy may be the optimal policy for a planner who respects investors' beliefs, as long as it endogenously provides insurance to investors through price changes. If markets are complete, a similar expression to the one in the static model quantifies welfare losses.

Overall, this paper provides a unified framework to understand financial transaction taxes and welfare. $^{2}$

\section{Related Literature}

This paper forms part of the literature that analyzes the Tobin (1978) proposal of introducing financial transaction taxes as a way to improve the societal performance of financial markets. Although Tobin's original exposition was focused on foreign exchange markets, it has become customary to refer to any tax on financial transactions as a "Tobin" tax. Stiglitz (1989) and Summers and Summers (1989) verbally lay out several arguments that support the implementation of a financial transaction tax; Ross (1989) takes the opposite view, highlighting many of the apparent contradictions of those verbal arguments. Roll (1989) and Schwert and Seguin (1993) also discuss related issues. Campbell and Froot (1994), several chapters in ul Haq, Kaul and Grunberg (1996) and Jones and Seguin (1997) are representative samples of empirical work in the area. See Habermeier and Kirilenko (2003) and McCulloch and Pacillo (2011) for recent discussions of the empirical literature.

My results are most closely related to Subrahmanyam (1998) and Dow and Rahi (2000), who also discuss formally welfare implications of financial transactions taxes. There are many differences between those papers and this one. The three most important ones are their use of quadratic taxes, noncompetitive investors and non-fundamental trading driven by noise traders, as opposed to the approach of this paper based on linear taxes, competitive markets and non-fundamental trading driven by belief disagreement.

The literature on transaction costs is also related, since a financial transaction tax is formally similar to a transaction cost. A non-exhaustive list of relevant papers is Amihud and Mendelson (1986), Constantinides (1986), Vayanos (1998), Lo, Mamaysky and Wang (2004), Liu (2005), Gârleanu and Pedersen (2012) and Buss and Dumas (2013). See Amihud, Mendelson and Pedersen (2005), Guasoni and Muhle-Karbe (2012) and Vayanos and Wang (2012) for recent surveys of portfolio choice models

\footnotetext{
${ }^{2}$ There are relevant issues regarding financial transaction taxes which this paper does not consider. For instance, there is no explicit role in this paper for liquidity, which could be naturally studied in a search environment, or for market power. This paper also does not analyze how transaction taxes affect wealth dynamics, endogenous learning dynamics and endogenous information acquisition. How transaction taxes worsen or improve welfare in these environments are important questions for future research.
} 
and equilibrium models with transactions costs.

This paper also forms part of the sizable literature on belief disagreement, speculation and trading. A non-exhaustive list of relevant papers is Miller (1977), Harrison and Kreps (1978), Hong and Stein (1999), Scheinkman and Xiong (2003), Geanakoplos (2010) and Simsek (2013a,b). In particular, Scheinkman and Xiong (2003) analyze the positive implications of a transaction tax in a model with belief disagreement; however, they do not make any statements about welfare. Hong and Stein (2007) and Xiong (2012) are two recent surveys of this line of work.

More broadly, in the sense that some trades are not driven by fundamental considerations, this paper also relates to the literature on noise trading. Fischer Black, in his 1985 AFA presidential address Noise, writes:

\section{"People who trade on noise are willing to trade even though from an objective point of view they would be better off not trading"}

Note that he makes a normative statement — better off — and refers to an "objective point of view" - as the planner in this paper does. A natural question after reading Black (1986) is whether there exists a policy which, from an objective point of view, can make investors better off. This paper shows that a transaction tax may be such a policy. ${ }^{3}$ However, the standard noise trading formulation, as in Grossman and Stiglitz (1980), Hellwig (1980), Diamond and Verrecchia (1981) or De Long et al. (1990), is not appropriate to study the welfare implications of a policy like a transaction tax. In particular, it is hard to understand how noise traders react to taxes and how to evaluate their welfare.

There has been some recent work on evaluating welfare under belief disagreement in financial markets. The most related paper to mine is Brunnermeier, Simsek and Xiong (2012), who propose a new criterion to evaluate welfare in models with belief heterogeneity: they assess efficiency by evaluating welfare under a convex combination of the beliefs of the investors in the economy. In particular, their criterion identifies outcomes related to zero-sum speculation, but they do not discuss realistic policy measures to limit these trades, which is the raison d'être of this paper. Whenever appropriate, I relate my results to their criterion. Gilboa, Samuelson and Schmeidler (2012) also present an alternative criterion that identifies negative sum betting situations and Posner and Weyl (2013) advocate for financial regulation grounded on price-theoretic analysis - that is exactly the goal of this paper. Also recently, Blume et al. (2013) propose a new criterion to calculate social welfare in a model with heterogeneous beliefs. Their planner defines a set of possible beliefs and then assess welfare under the worst case scenario among all belief assignments. Their results relate to the Friedman (1953) selection hypothesis, in the sense that the long-run behavior of the model is crucial to determine welfare. ${ }^{4}$ They discuss several restrictions on trading, but do not characterize optimal policies.

Lastly, this paper is related to the growing literature on behavioral welfare economics. The emphasis on the envelope theorem is reminiscent of Akerlof and Yellen (1985), which analyze how

\footnotetext{
${ }^{3}$ Chapter 7 of Shleifer (2000) presents several thoughts along these lines. He states that two relevant open questions in behavioral finance are "How can noise trading be discouraged without discouraging arbitrage?" and "Who should regulate securities markets and how?". This paper provides coherent answers to both questions.

${ }^{4}$ See Blume and Easley (2010) for a recent survey of the literature on survival and selection in financial markets.
} 
individual near-rational behavior can have first-order effects in the aggregate. My paper is more directly related to O'Donoghue and Rabin (2006), in which commitment problems due to hyperbolic discounting for a fraction of investors create room for public intervention through taxation. Sandroni and Squintani (2007) and Spinnewijn (2012) analyze behavioral biases in insurance markets and unemployment search respectively. See Bernheim (2009) and Mullainathan, Schwartzstein and Congdon (2012) for surveys of recent work in behavioral welfare economics and Bernheim and Rangel (2009) for an alternative approach to welfare with behavioral agents.

Outline Section 2 introduces and solves the baseline model and section 3 executes the normative analysis, solving for the optimal tax. Section 4 provides intuition for the main results with several numerical examples and section 5 presents more extensions within the baseline static/CARA framework. Section 6 generalizes the results derived in the baseline model to multiple periods, a general utility specification and an arbitrary form of disagreement. Section 7 separately extends the results to a Walrasian production economy and a Hayekian production economy. Section 8 concludes. Appendix A contains derivations and proofs and appendix $\mathrm{B}$ additional results and extensions.

\section{Baseline Model}

This section presents a static model with CARA utility and investors who disagree only about expected returns. When transaction taxes are zero, this environment is identical to Lintner (1969), who relaxes the standard CAPM by allowing for heterogeneous beliefs among investors. ${ }^{5}$

\subsection{Environment}

Investors Time is discrete, there are two dates $t=\{1,2\}$ and there is a unit measure of investors. Investors are indexed by $i$ and they are distributed according to a probability distribution $F$; therefore $\int d F(i)=1$. The distribution $F$ can be discrete, continuous or a mixture of both.

Investors choose their portfolio allocation at $t=1$ and consume at $t=2$. They maximize expected utility with preferences that feature constant absolute risk aversion. Therefore, each investor maximizes:

$$
\mathbb{E}_{i}\left[U_{i}\left(W_{2 i}\right)\right] \text { with } \quad U_{i}\left(W_{2 i}\right)=-e^{-A_{i} W_{2 i}},
$$

where (1) already imposes that investors consume all terminal wealth, that is $C_{2 i}=W_{2 i}$. The parameter $A_{i}>0$, which represents the coefficient of absolute risk aversion $A_{i} \equiv-\frac{U_{i}^{\prime \prime}}{U_{i}^{\prime}}$, can vary freely in the distribution of investors. The index $i$ in the expectation is necessary because every investor holds heterogeneous beliefs about expected returns. In this section, investors only disagree about the first moment of the distribution of returns.

\footnotetext{
${ }^{5}$ See Fama (1976), Jarrow (1980) or Fama and French (2007) for related work along these lines.
} 
Market structure and beliefs There exists a riskless asset in elastic supply with a (exogenously determined) gross interest rate $R=16^{6}$ There is a single risky asset with exogenously fixed supply $Q \geq 0$. The price of the risky asset at $t=1$ is denoted by $P_{1}$ and is quoted in terms of an underlying good (dollar), which acts as numeraire. The initial holdings of the risky asset at $t=1$, given by $X_{0 i}$, are arbitrary across the distribution of investors. All together, the investors must hold the total supply $Q$, therefore $\int X_{0 i} d F(i)=Q$. Investors face no constraints when choosing portfolios: they can borrow and short sell freely.

The risky asset yields a dividend $D$ in period 2. The objective (true) distribution of the dividend $D$ paid by the risky asset is a normal with mean $\mathbb{E}[D]$ and strictly positive variance $\mathbb{V a r}[D]$, that is:

$$
D \sim N(\mathbb{E}[D], \mathbb{V a r}[D])
$$

Investors hold heterogeneous dogmatic beliefs about $D$. An investor $i$ believes that $D$ is normally distributed with mean $\mathbb{E}_{i}[D]$ and variance $\mathbb{V a r}[D]$, that is:

$$
D \sim_{i} N\left(\mathbb{E}_{i}[D], \mathbb{V a r}[D]\right)
$$

Investors do not learn from each other, or from the price, and agree to disagree in the Aumann (1976) sense. For now, the pattern of belief disagreement is arbitrary across the distribution of investors. Nothing prevents investors from having correct beliefs; investors with correct beliefs can represent market makers or (limited) arbitrageurs.

Hedging needs Every investor has a stochastic endowment at $t=2$, denoted by $E_{2 i}$, which is normally distributed and potentially correlated with $D$. This endowment captures the fundamental risks associated with the normal economic activity of each investor. The exposure of an investor $i$ to this underlying risk is captured by the covariance $\operatorname{Cov}\left[E_{2 i}, D\right]$. This covariance is a main determinant of the optimal portfolio allocation. For now, investors hold correct beliefs about $\operatorname{Cov}\left[E_{2 i}, D\right]$. Without loss of generality, I assume that $\mathbb{E}\left[E_{2 i}\right]-\frac{A_{i}}{2} \operatorname{Var}\left[E_{2 i}\right]=0$ and normalize the initial endowment $E_{1 i}$ to zero for all investors.

Trading motives Summing up, there are four reasons to trade in this model:

(a) Different hedging needs: captured by $\operatorname{Cov}\left[E_{2 i}, D\right]$

(b) Different risk aversion: captured by $A_{i}$

(c) Different initial asset holdings: captured by $X_{0 i}$

(d) Different beliefs: captured by $\mathbb{E}_{i}[D]$

The first three correspond to fundamental reasons for trading: sharing risks among investors, transferring risks to those more willing to bear them or trading for life cycle or liquidity needs. Trading on different beliefs is the single source of non-fundamental trading in the model.

\footnotetext{
${ }^{6}$ The analysis when $R \neq 1$ is straightforward. Appendix B re-derives propositions 1 and 2 when $R \neq 1$.
} 


\subsubsection{Policy instrument: a linear financial transaction tax}

Tax definition This paper adopts the Ramsey approach of solving for an optimal policy under a restricted set of instruments. The single policy instrument available to the planner is an anonymous linear financial transaction tax $\tau$ paid per dollar traded in the risky asset. In particular, a change in the asset holdings of the risky asset $\left|X_{1 i}-X_{0 i}\right|$ at a price $P_{1}$ faces a total tax in terms of the numeraire, due at the same time the transaction occurs, for both buyers and sellers, of:

$$
\tau\left|P_{1}\right|\left|\Delta X_{1 i}\right|
$$

where $\left|\Delta X_{1 i}\right| \equiv\left|X_{1 i}-X_{0 i}\right|$. Total tax revenue generated by that transaction is thus $2 \tau\left|P_{1}\right|\left|\Delta X_{1 i}\right|$. I restrict $\tau$ to be in a closed interval $[\underline{\tau}, \bar{\tau}]$ such that $-1<\underline{\tau}$ and $\bar{\tau}<1$. In general, the use of the absolute value for the price in (2) is needed because asset prices can be negative in this model, as they are in many markets for derivative contracts. I restrict soon the analysis to situations with strictly positive prices.

Analytically, nothing prevents the tax to be negative (a subsidy). However, an anonymous linear trading subsidy can never be implemented: investors would continuously exchange assets, making infinite profits. Therefore, when I refer to subsidies in this paper, I implicitly assume that the planner is able to rule out this type of "wash trades".

Linearity and anonymity I restrict the analysis to linear taxes with the intention of being realistic. There is a powerful argument for the use of linear (as opposed to non-linear) taxes in this environment: linear taxes are the most robust to sophisticated trading schemes. ${ }^{7}$ For example, a lump-sum tax per trade creates incentives to submit a single large order; alternatively, quadratic taxes create incentives to split orders into infinitesimal pieces. These concerns, which are shared with other non-linear tax schemes, are particularly relevant for financial transaction taxes, given the high degree of sophistication of many players in financial markets and the negligible costs of splitting orders given modern information technology. ${ }^{8}$

I assume that transaction taxes must apply across-the-board to all market participants and cannot be conditioned on individual characteristics. This implies that the planner's problem is a second-best problem. A planner with the ability to distinguish good trades from bad trades could achieve the first-best by taxing harmful trades on an individual basis: this is an implausible assumption. More generally, any policy which allows the planner to directly target bad trades would improve welfare see subsection 5.5 for a characterization of the first-best in this model and appendix B for a more detailed discussion of policy instruments.

No tax avoidance Furthermore, I assume that investors cannot avoid paying transaction taxes, either by trading secretly, within an intermediary that cancels opposites trades, or by moving to a different

\footnotetext{
${ }^{7}$ The same absence of arbitrage condition that underlies the law of one price in financial markets - see Duffie (2001) or Cochrane (2005) - effectively constrains the optimal tax to be linear.

${ }^{8}$ I see no tension in assuming that investors hold heterogeneous (and wrong) beliefs at the same time that they are capable of exploiting non-linearities in the tax code. Forecasting expected returns is normally considered a much harder problem than implementing trades in such a way that tax liabilities are minimized.
} 
exchange. This behavior is optimal when the penalties from evasion are sufficiently large, provided the taxable event is appropriately defined. These issues are critical for implementing financial transaction taxes in practice, but I abstract from them in this paper.

Revenue rebate and lump-sum redistribution Lastly, since this paper focuses on the corrective (Pigovian) effects of transaction taxes and not on the ability of this tax to raise fiscal revenue, I assume that tax proceeds are rebated lump-sum to investors. ${ }^{9}$ Under CARA utility, the rebate that each particular investor receives is irrelevant to determine trading behavior, although variations in the individual level of the transfers change wealth and marginal utility. Only to simplify the analysis, I assume that every (group of) investor(s) $i$ receives a rebate $T_{1 i}$ equal to his (their) own tax liability, that is $T_{1 i}=\tau\left|P_{1}\right|\left|\Delta X_{1 i}\right|$. Investors do not internalize the rebate, since they are assumed to be small.

To sharpen many results, I allow for the possibility that the planner is able to redistribute wealth across investors in a lump-sum way at an ex-ante stage - I'll be explicit about this fact when required. This assumption allows the planner to focus on efficiency considerations.

This paper has purposefully nothing to say about how to spend any tax revenue generated.

Investors' budget constraints Hence, consumption/wealth of a given investor $i$ at $t=2$ is composed of the stochastic endowment $E_{2 i}$, the stochastic payoff of the risky asset $X_{1 i} D$ and the return on the investment in the riskless asset. This includes the net purchase or sale of the risky asset $\left(X_{0 i}-X_{1 i}\right) P_{1}$, the total tax liability $-\tau\left|P_{1}\right|\left|\Delta X_{1 i}\right|$ and the lump-sum transfer $T_{1 i}$. It can be written as:

$$
W_{2 i}=E_{2 i}+X_{1 i} D+\left(X_{0 i} P_{1}-X_{1 i} P_{1}-\tau\left|P_{1}\right|\left|\Delta X_{1 i}\right|+T_{1 i}\right)
$$

\subsubsection{Equilibrium definition}

A competitive equilibrium with taxes is defined as a portfolio allocation $X_{1 i}$ for every investor, a price $P_{1}$ and set of lump-sum transfers $T_{1 i}$ such that: a) investors maximize expected utility in $X_{1 i}$, subject to their budget constraint $(3)$; b) the price $P_{1}$ is such the market for the risky asset clears, that is $\int X_{1 i} d F(i)=Q$ and c) tax revenues are rebated in a lump-sum fashion to investors, that is $\int T_{1 i} d F(i)=\tau\left|P_{1}\right| \int\left|\Delta X_{1 i}\right| d F(i)$.

Positive prices Before characterizing the equilibrium of the model, I make an assumption to guarantee that the equilibrium price of the risky asset is strictly positive. This assumption simplifies the number of cases to consider.

Assumption 1. [PP] (Positive prices) The (perceived) moments of the distribution of dividends are such that equilibrium prices are always positive. A sufficient condition is that the (perceived) expected dividend is large

\footnotetext{
${ }^{9}$ Broadly defined, there are two types of taxes: those levied with the aim of raising revenue and those levied with the aim of correcting distortions. This paper only analyzes corrective taxes. Sandmo (1975) shows that corrective taxes are additive with respect to the optimal revenue raising taxes; see also Kopczuk (2003). I also do not consider in this paper the additional benefits/losses of corrective policies generated by "double-dividend" arguments. Those arguments, surveyed by Goulder (1995) in the context of environmental taxation, apply directly to the case of transaction taxes.
} 
enough when compared to the risk bearing capacity of every investor, that is:

$$
\mathbb{E}_{i}[D]>A_{i}\left(\operatorname{Cov}\left[E_{2 i}, D\right]+\mathbb{V} a r[D] Q\right), \quad \forall i
$$

The sufficient condition given in (4) is not at all a necessary condition. Any combination of expected payoffs and risk bearing capacity which makes the equilibrium price, given in equation (7) below, positive for the relevant range of $\tau$ would work. From now on, assumption [PP] holds and I use $P_{1}$ instead of $\left|P_{1}\right|$ - subsection 7.2 deals with potentially negative prices.

\subsection{Equilibrium characterization}

I initially solve for investors portfolio demands given prices. Subsequently, I characterize the equilibrium price and allocations.

Investor's problem Because of CARA utility and normality of returns, the demand for the risky asset of every investor is derived by solving a mean-variance problem in $X_{1 i}$. In particular, after getting rid of terms that do not affect the maximization problem, investors solve:

$$
\max _{X_{1 i}}\left[\mathbb{E}_{i}[D]-A_{i} \operatorname{Cov}\left[E_{2 i}, D\right]-P_{1}\right] X_{1 i}-\tau P_{1}\left|\Delta X_{1 i}\right|-\frac{A_{i}}{2} \mathbb{V a r}[D] X_{1 i}^{2}
$$

The first order condition of this problem yields the following demand for the risky asset: ${ }^{10}$

$$
X_{1 i}=\left\{\begin{array}{lll}
\frac{\mathbb{E}_{i}[D]-A_{i} \operatorname{Cov}\left[E_{2 i}, D\right]-P_{1}(1+\tau)}{A_{i} \mathbb{V a r}[D]} ; & \Delta X_{1 i}>0 & \text { Buying } \\
X_{0 i} ; & \Delta X_{1 i}=0 & \text { No Trade } \\
\frac{\mathbb{E}_{i}[D]-A_{i} \operatorname{Cov}\left[E_{2 i}, D\right]-P_{1}(1-\tau)}{A_{i} \mathbb{V a r}[D]} ; & \Delta X_{1 i}<0 & \text { Selling }
\end{array}\right.
$$

The optimal portfolio choice is represented in figure 1, which shows the net change in asset holdings $\Delta X_{1 i}$ for an investor $i$ as a function of his initial asset holdings $X_{0 i}$.

The presence of linear transaction taxes modifies the optimal portfolio allocation in two dimensions. First, a transaction tax is reflected as a higher price $P_{1}(1+\tau)$ paid by buyers and a lower price $P_{1}(1-\tau)$ received by sellers. Hence, the tax reduces the amount of trading $\left|\Delta X_{1 i}\right|$ at the intensive margin for both buyers and sellers. Second, in addition to a reduction in trading at the margin, a linear tax implies that some investors decide not to trade altogether, creating an inaction region. In particular, when taxes are positive, for a given price $P_{1}$, the no-trade region for an individual investor $i$ is given by:

$$
\frac{\mathbb{E}_{i}[D]-A_{i} \operatorname{Cov}\left[E_{2 i}, D\right]-P_{1}(1+\tau)}{A_{i} \mathbb{V} \operatorname{Var}[D]}<X_{0 i}<\frac{\mathbb{E}_{i}[D]-A_{i} \operatorname{Cov}\left[E_{2 i}, D\right]-P_{1}(1-\tau)}{A_{i} \mathbb{V} \operatorname{Var}[D]}
$$

If the initial holdings of the risky asset $X_{0 i}$ are not too far from the optimal allocation without taxes $\frac{\mathbb{E}_{i}[D]-A_{i} \operatorname{Cov}\left[E_{2 i}, D\right]-P_{1}}{A_{i} \mathbb{V} \operatorname{ar}[D]}$, an investor decides not to trade. When $\tau=0$, the no-trade region ceases to exist.

\footnotetext{
${ }^{10}$ The problem solved by investors is convex, so first order conditions are necessary and sufficient to characterize optimal asset holdings. Furthermore, this fact, combined with the absence of income effects, are sufficient to guarantee existence and uniqueness of the competitive equilibrium given a tax $\tau$.
} 


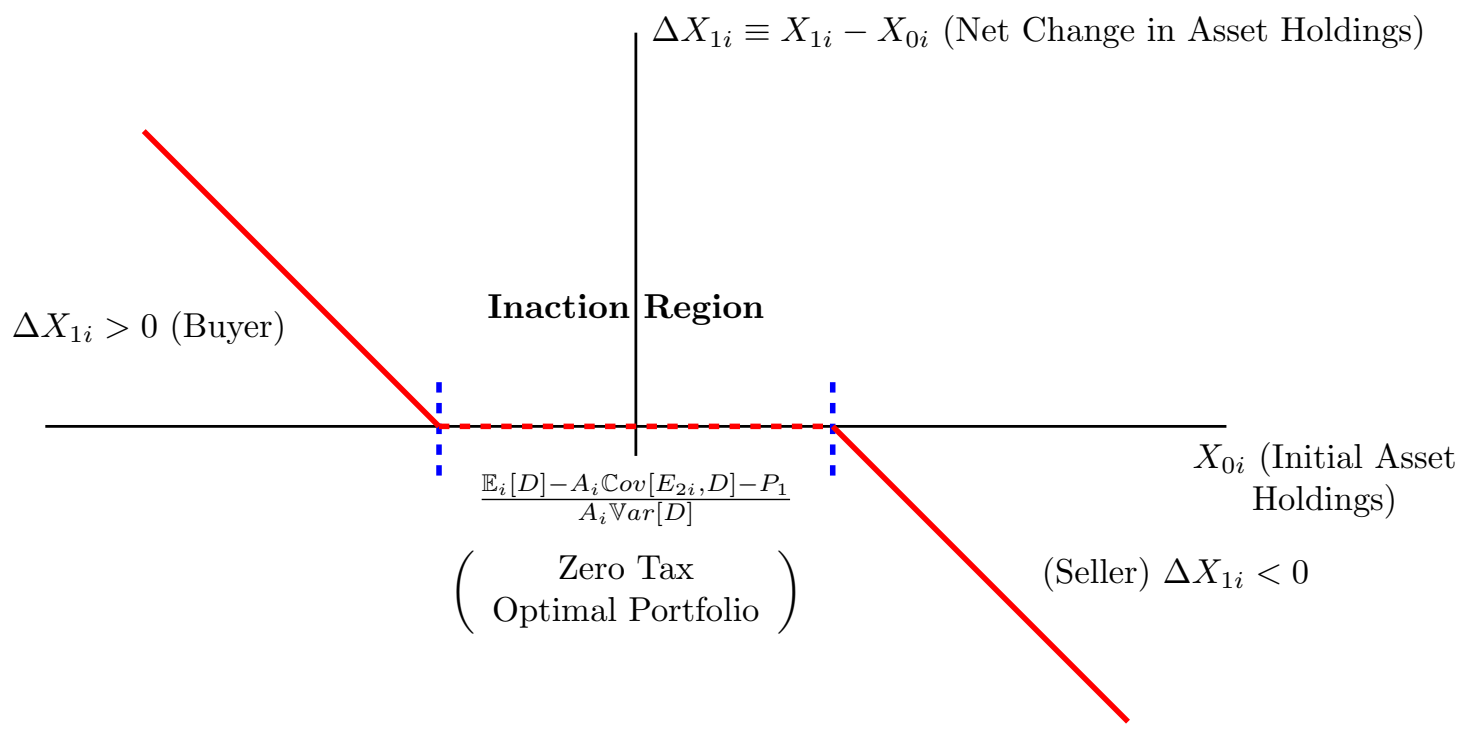

Figure 1: Optimal portfolio allocation/Inaction region

Linear transaction costs naturally create inaction regions. The envelope theorem, which plays an important role when deriving the optimal tax results, is also key to generate the inaction region, as originally shown in Constantinides (1986). Intuitively, an investor with initial asset holdings close to his optimum experiences a second-order gain from a marginal trade but suffers a first-order loss when a linear tax is present. It is thus optimal for him not to trade. If, for instance, taxes were quadratic instead of linear, the marginal welfare loss induced by the tax around the optimum would also be second-order, eliminating the inaction region.

Equilibrium price Given the optimal portfolio allocation derived in (5) and the market clearing condition $\int X_{1 i} d F(i)=Q$, the equilibrium price of the risky asset can be written as:

$$
P_{1}=\frac{\int_{i \in \mathcal{T}}\left(\frac{\mathbb{E}_{i}[D]}{\mathcal{A}_{i}}-A\left(\operatorname{Cov}\left[E_{2 i}, D\right]+\mathbb{V a r}[D] X_{0 i}\right)\right) d F(i)}{1+\tau \int_{i \in \mathcal{T}} \frac{\operatorname{sgn}\left(\Delta X_{1 i}\right)}{\mathcal{A}_{i}} d F(i)}
$$

where $A \equiv\left(\int_{i \in \mathcal{T}} \frac{1}{A_{i}} d F(i)\right)^{-1}$ is the harmonic mean of risk aversion coefficients for active investors and $\mathcal{A}_{i} \equiv \frac{A_{i}}{A}$ is the quotient between the risk aversion coefficient of investor $i$ and the harmonic mean. I denote by $i \in \mathcal{T}$ that all integrations are made only over investors who are actively trading in equilibrium. ${ }^{11}$ Intuitively, only marginal investors determine directly the equilibrium price. I use $\operatorname{sgn}(\cdot)$ to denote the sign function.

The numerator of the equilibrium price has two components. The first one is a risk aversion weighted average of the expected payoff of the risky asset. The second one is a risk premium, determined by the product of price and quantity of risk. The price of risk is given by the harmonic mean of risk aversion coefficients $A$. The quantity of risk consists of two terms. The first one is the

\footnotetext{
${ }^{11}$ Equation (6) defines explicitly the set of active investors. Because the area of integration implied by $i \in \mathcal{T}$ depends on the equilibrium price, equation (7) provides an implicit characterization of $P_{1}$.
} 
sum of covariances of the risky asset with the endowments $\int_{i \in \mathcal{T}} \operatorname{Cov}\left[E_{2 i}, D\right] d F(i)$. The second one is the product of the the variance of the risky asset $\mathbb{V a r}[D]$ with the number of shares initially held by investors $\int_{i \in \mathcal{T}} X_{0 i} d F(i)$.

The tax rate $\tau$ only appears directly in the denominator of the equilibrium price; it also appears indirectly in the limits of integration. When $\tau=0$, the numerator of (7) completely determines the equilibrium price. The following lemma characterizes how the equilibrium price varies with $\tau$.

Lemma 1. (Effect of $\tau$ on prices) An increase in the transaction tax can increase, reduce or keep constant the equilibrium price. When $\int_{i \in \mathcal{T}} \frac{\operatorname{sgn}\left(\Delta X_{1 i}\right)}{\mathcal{A}_{i}} d F(i)$ is positive/negative/zero, $\frac{d P_{1}}{d \tau}$ is negative/positive/zero.

Proof. See appendix A.

Intuitively, a marginal change in $\tau$ reduces both supply and demand for the risky asset, so the relative strength of supply and demand price derivatives determines whether $P_{1}$ increases or decreases in $\tau$. The key determinant of $\frac{d P_{1}}{d \tau}$ is $\int_{i \in \mathcal{T}} \frac{\operatorname{sgn}\left(\Delta X_{1 i}\right)}{A_{i}} d F(i)$, which is the weighted difference between buying and selling marginal responses to price changes when $\tau=0$ - see appendix A for more details. When this term is positive, increasing $\tau$ reduces the buying pressure by more than the selling pressure, reducing the equilibrium price, and viceversa. When total demand and supply price derivatives are symmetric - this occurs, for instance, when all investors have identical risk aversion coefficients and the mass of buyers equals the mass of sellers - the equilibrium price is independent of the tax. This is an interesting benchmark. It shows that, if supply and demand are symmetric, transaction taxes should not affect equilibrium prices.

The result that equilibrium prices can increase in the level of transaction costs may seem counterintuitive, but it is well-known in the general equilibrium transaction costs literature. It is originally derived by Vayanos (1998).

Two final remarks about equilibrium prices. First, I show in appendix B that the equilibrium price $P_{1}$ is differentiable in $\tau$ almost everywhere. When the distribution $F$ is everywhere continuous, $P_{1}$ is continuously differentiable. Second, the equilibrium price is only well defined when there is trade in equilibrium. For a sufficiently large $\tau$, it is optimal for every investor to keep his initial shares; trade stops and there exists no equilibrium price.

Equilibrium portfolio allocations The allocation of the risky asset in equilibrium can be found by substituting the equilibrium price, expressed in (7), into the portfolio demand by every investor, expressed in (5). The following property about equilibrium allocations is useful for the subsequent welfare analysis. I denote the set of active buyers by $\mathcal{B}$ and the set of active sellers by $\mathcal{S}$ - the identity of the investors in each of the sets varies with $\tau$.

\section{Lemma 2. (Effect of $\tau$ on allocations)}

a) An increase in the transaction tax $\tau$ reduces the equilibrium asset holdings for every buyer, that is, $\frac{d X_{1 i}}{d \tau}<0$ for $i \in \mathcal{B}$, and increases the equilibrium asset holdings for every seller, that is, $\frac{d X_{1 i}}{d \tau}>0$, for $i \in \mathcal{S}$.

b) An increase in the transaction tax $\tau$ reduces volume, that is, $\int_{i \in \mathcal{B}} \frac{d X_{1 i}}{d \tau} d F(i)<0$. 
Proof. See appendix A.

Intuitively, when $\tau$ increases, buyers buy fewer shares of the risky asset and sellers sell fewer shares of the risky asset. This result is intuitive but not immediately obvious, because equilibrium allocations $X_{1 i}\left(\tau, P_{1}\right)$ depend on $\tau$ both directly and through the equilibrium price. The equilibrium response of $X_{1 i}$ to a change in the tax $\tau$ is thus given by:

$$
\frac{d X_{1 i}}{d \tau}=\frac{\partial X_{1 i}}{\partial \tau}+\frac{\partial X_{1 i}}{\partial P_{1}} \frac{d P_{1}}{d \tau}
$$

As shown in (5), the first term $\frac{\partial X_{1 i}}{\partial \tau}$ is negative for buyers and positive for sellers. The second term

$\frac{\partial X_{1 i}}{\partial P_{1}}$ is negative for both buyers and sellers and, as stated in lemma $1, \frac{d P_{1}}{d \tau}$ can have any sign. Lemma 2 guarantees that the effect of price changes in equilibrium allocations is not strong enough, concluding that $\frac{d X_{1 i}}{d \tau}$ and $\frac{\partial X_{1 i}}{\partial \tau}$ have the same sign. The absence of income effects, implied by the CARA specification, is crucial to derive this result. The result about equilibrium volume follows directly. Finally, because $X_{1 i}$ is continuous in $\tau$ and $P_{1}$, the equilibrium allocations inherit the differentiability properties of $\frac{d P_{1}}{d \tau}$.

\section{Summary of positive results}

Summing up, the positive analysis of the model concludes:

(a) Increasing transaction taxes always reduces volume in equilibrium. At the margin, every buyer buys fewer shares and every seller sells fewer shares.

(b) Increasing transaction taxes can increase, reduce or keep unchanged asset prices and returns. The same conclusion applies to price volatility in a dynamic context.

The existing empirical evidence supports the positive predictions of the model. A robust empirical fact is that volume goes down (up) after an increase (reduction) on transaction taxes/costs. However, the evidence of the effect on prices is mixed. Some studies find an increase in price volatility, but others find no significant changes or even a reduction. Asset prices usually fall at impact following a tax increase, but seem to recover over time. See Roll (1989), Umlauf (1993), the review articles by Campbell and Froot (1994) and Habermeier and Kirilenko (2003) or the recent survey by McCulloch and Pacillo (2011) for evidence.

\section{Welfare Analysis: Optimal Financial Transaction Tax}

After characterizing price and equilibrium allocations for a given financial transaction tax, I solve for the welfare maximizing value of $\tau$.

\subsection{Welfare criterion}

Social welfare function The major contribution of this paper resides in the welfare analysis of the policy of taxing financial transactions. To carry out this task, I must take a stand on how to evaluate 
social welfare when investors hold heterogeneous beliefs. ${ }^{12}$ I approach this problem by making two assumptions about the planner's social welfare function.

First, the planner maximizes an arbitrarily weighted sum of utilities of the investors. This approach simply characterizes the Pareto frontier of the economy. This is a standard approach in normative economics and should raise no particular concerns.

Second, the planner calculates individual welfare using a different probability distribution about payoffs than the one perceived by each investor. In practice, I initially assume that the planner maximizes welfare using the true probability distribution. Subsequently, I point out under which circumstances the optimal policy does not depend on the distribution used by the planner — in those cases, the planner only assumes that there exists a single distribution.

Two different questions emerge here. First, does the planner respect subjective beliefs when calculating social welfare? The answer to this question is negative. The planner does not respect subjective beliefs. In that sense, the approach followed by the planner is paternalistic. Second, does the planner need to know the true distribution of the dividend process? The answer to this question is "it depends". Under certain (plausible) circumstances - specified below -, the planner does not need to know the true probability distribution to implement the optimal policy. In those cases, despite being aware of the existence of disagreement, the planner does not enjoy any informational advantage with respect to market participants. Any criticism about paternalistic policies on the grounds that the planner must have better information than economic agents does not apply then.

Motivation Two arguments are consistent with the welfare criterion adopted in this paper. The first one relies on the idea that rational investors cannot agree to disagree when their posteriors are common knowledge. ${ }^{13}$ How can the planner respect investors' beliefs when they are inconsistent with one another? If we assume that there is a single truth but different investors hold different beliefs, all of them (but one) must be wrong.

Alternatively, a veil of ignorance interpretation is also consistent with this welfare criterion. If investors acknowledge that they may wrongly hold different beliefs, they would happily implement, at an ex-ante stage, a tax policy that curtails trading. However, once an investor observes his beliefs and regards them as correct, he will perceive that any tax policy is welfare reducing.

\subsection{Optimal tax policy}

After presenting the welfare criterion used by the planner, I characterize the optimal tax. Social welfare, denoted by $V$, can thus be written as the sum of the indirect utility for every investor $i$, under the distribution of returns used by the planner, that is:

$$
V(\tau)=\int \lambda_{i} V_{i} d F(i)
$$

\footnotetext{
${ }^{12}$ This is a controversial issue. In addition to the work discussed on the literature section, see Kreps (2012) for some reflections on this topic.

${ }^{13}$ Morris (1995) has a thorough discussion of the common prior assumption. See Fudenberg and Tirole (1991) or Brunnermeier (2001) for textbook expositions.
} 
where $\lambda_{i}>0$ is the welfare weight assigned by the planner to an investor $i$.

$V_{i}$ denotes the indirect utility for an investor $i$ from the planner's perspective, which can be expressed as:

$$
V_{i} \equiv \mathbb{E}\left[U_{i}\left(W_{2 i}\right)\right]=-e^{-A_{i} \hat{V}_{i}} \quad \text { with } \quad \hat{V}_{i} \equiv \mathbb{E}\left[W_{2 i}\left(X_{1 i}, P_{1}\right)\right]-\frac{A_{i}}{2} \mathbb{V a r}\left[W_{2 i}\left(X_{1 i}, P_{1}\right)\right],
$$

where $W_{2 i}$ is a function of the portfolio allocation optimally chosen by investors $X_{1 i}$ and the equilibrium price $P_{1} . \hat{V}_{i}$ is the certainty equivalent from the planner's perspective. Note that the expectation used to calculate the indirect utility $V_{i}$ does not have an individual subscript $i$, because it is taken under the planner's distribution.

Substituting $W_{2 i}$ explicitly and using the fact that tax proceeds are rebated lump-sum to every investor, i.e., $T_{i}=\tau P_{1}\left|\Delta X_{1 i}\right|$, the indirect utility of an investor $i$ from the planner's perspective can be written as:

$$
V_{i}=-e^{-A_{i}\left(\left(\mathbb{E}[D]-A_{i} \operatorname{Cov}\left[E_{2 i}, D\right]-P_{1}\right) X_{1 i}+P_{1} X_{0 i}-\frac{A_{i}}{2} \operatorname{Var}[D]\left(X_{1 i}\right)^{2}\right)},
$$

where $X_{1 i}$ is chosen by every investor according to the first order condition described in (5). Note that both $X_{1 i}$ and $P_{1}$ are in principle functions of $\tau$.

The optimal tax $\tau^{*}$ is defined by $\tau^{*}=\arg \max _{\tau} V(\tau)$. The first step to characterize $\tau^{*}$ is to find $\frac{d V_{i}}{d \tau}$, the marginal effect of varying taxes on the indirect utility of investor $i$ from the planner's perspective. This is given by:

$$
\frac{d V_{i}}{d \tau}=A_{i} e^{-A_{i} \hat{V}_{i}} \frac{d \hat{V}_{i}}{d \tau}=\mathbb{E}\left[U_{i}^{\prime}\left(W_{2 i}\right)\right] \frac{d \hat{V}_{i}}{d \tau}
$$

The value of $\frac{d V_{i}}{d \tau}$ is given by the (dollar) change in the certainty equivalent $\frac{d \hat{V}_{i}}{d \tau}$, valued at the expected marginal utility of wealth from the planner's perspective $\mathbb{E}\left[U_{i}^{\prime}\left(W_{2 i}\right)\right]$. The marginal change in the certainty equivalent $\frac{d \hat{V}_{i}}{d \tau}$ can be expressed as:

$$
\begin{aligned}
\frac{d \hat{V}_{i}}{d \tau} & =\left[\mathbb{E}[D]-A_{i} \operatorname{Cov}\left[E_{2 i}, D\right]-P_{1}-A_{i} \mathbb{V} \operatorname{ar}[D] X_{1 i}\right] \frac{d X_{1 i}}{d \tau}-\Delta X_{1 i} \frac{d P_{1}}{d \tau} \\
& =\left[\left(\mathbb{E}[D]-\mathbb{E}_{i}[D]\right)+\operatorname{sgn}\left(\Delta X_{1 i}\right) P_{1} \tau\right] \frac{d X_{1 i}}{d \tau}-\Delta X_{1 i} \frac{d P_{1}}{d \tau}
\end{aligned}
$$

The expression for $\frac{d \hat{V}_{i}}{d \tau}$ consists of two terms. The first term captures how welfare varies through changes in the portfolio allocation. Intuitively, a change in the allocation $\frac{d X_{1 i}}{d \tau}$ changes the certainty equivalent of investor $i$ only when there exists a wedge in his portfolio demand. In this case, a first wedge arises when $\mathbb{E}[D] \neq \mathbb{E}_{i}[D]$, due to the belief distortion. A second wedge arises when the tax is strictly positive, that is when $\operatorname{sgn}\left(\Delta X_{1 i}\right) P_{1} \tau \neq 0$. In a model with homogenous beliefs and no taxes, a marginal increase in $\tau$ has no effect on welfare through changes in the portfolio allocation. The second term in $\frac{d \hat{V}_{i}}{d \tau}$ captures terms-of-trade effects. If $P_{1}$ increases with $\tau$, the buyers of the risky asset are worse off, since they now buy a more expensive asset: the terms-of-trade of their purchase have worsened. However, sellers are better off, since they receive a higher price for their sale. The opposite occurs when prices decrease with $\tau$.

The first line in (9) follows directly by taking derivatives on $V_{i}$. The second line follows by substituting $X_{1 i}$ with the investors optimal portfolio choice, given by (5). The derivation of (9) also uses an envelope condition for the extensive margin choice between trading and not trading, which is 
made optimally. When an investor decides not to trade, the marginal change $\frac{d X_{1 i}}{d \tau}$ is zero and there are no terms-of-trade-effects, because $X_{1 i}=X_{0 i}$. Hence, a marginal tax change has no effect at all on the welfare of those investors who decide not to trade.

Assumptions Before characterizing the main results of this section, I introduce assumptions [NR] and [OBPS].

Assumption 2. [NR] (No Redistribution) The product of the social welfare weight $\lambda_{i}$ with the expected marginal utility under the planner's probability distribution $\mathbb{E}\left[U_{i}^{\prime}\left(W_{2 i}\right)\right]$ does not vary across the distribution of investors, that is:

$$
\lambda_{i} \mathbb{E}\left[U_{i}^{\prime}\left(W_{2 i}\right)\right] \text { is constant for all investors }
$$

Assumption [NR] rules out any redistributional considerations regarding optimal tax policy, allowing the planner to exclusively focus on the efficiency of the portfolio allocation - it is equivalent to assuming quasilinear preferences in a commodity taxation problem. When applied to this CARA/Normal model, social welfare becomes the (unweighted) sum of investors' certainty equivalents. Under this assumption, social welfare is independent of the way in which the planner chooses to rebate tax revenue to investors.

Assumption [NR] shares the spirit of the "large family" formulation in Lucas (1990) and it is implicitly made in any macroeconomic model featuring a representative agent. To implement assumption [NR] in practice given a set of welfare weights, the planner must use ex-ante lump-sum transfers, contingent on the level of $\tau$, in order to appropriately vary expected marginal utilities.

Assumption 3. [OBPS] (Optimists are mostly Buyers/Pessimists are mostly Sellers) The cross-sectional covariance of expected payoffs $\mathbb{E}_{i}[D]$ with respect to marginal changes in equilibrium allocations $\frac{d X_{1 i}}{d \tau}$ at $\tau=0$ is negative, that is:

$$
\operatorname{Cov}_{F}\left(\mathbb{E}_{i}[D],\left.\frac{d X_{1 i}}{d \tau}\right|_{\tau=0}\right)=\left.\int \mathbb{E}_{i}[D] \frac{d X_{1 i}}{d \tau}\right|_{\tau=0} d F(i)<0,
$$

where $\operatorname{Cov}_{F}[\cdot]$ denotes the covariance in the cross-section of the distribution of investors and the equality follows by using market clearing.

Assumption [OBPS] holds when optimistic investors happen to be (on average) the buyers of the risky asset and pessimistic investors happen to be the sellers. If all trading is driven by belief disagreement, assumption [OBPS] always holds — in that case, optimists buy and pessimists sell. However, because investors also trade due to fundamental reasons, it is possible that optimistic investors happen to be net sellers in equilibrium. For example, this situation occurs when workers are overoptimistic about the returns of their own companies and do not sufficiently hedge their implicit labor market risk. ${ }^{14}$ Assumption [OBPS] implies that this phenomenon is not too prevalent in the laissez-faire economy.

\footnotetext{
${ }^{14}$ Benartzi (2001), Massa and Simonov (2006) and Døskeland and Hvide (2011) provide evidence of this phenomenon. See Campbell (2006) for a discussion of related issues.
} 
Assumption [OBPS] can be justified empirically and theoretically. First, I appeal to the evidence suggesting that belief distortions are an important driver of the large amount of trading volume observed in financial markets. See, for instance, the surveys by Hong and Stein (2007) or Barber and Odean (2013). Second, in appendix B, I justify assumption [OBPS] exclusively on theoretical grounds. Intuitively, because an optimistic (pessimistic) investor who expects a high (low) payoff is more likely to be a buyer (seller) in equilibrium, unless the pattern of fundamental trading specifically counteracts this force, we should expect the covariance to be negative. In other words, if fundamental trading is orthogonal to non-fundamental trading, we expect [OBPS] to hold.

Finally, an example can help clarify how assumption [OBPS] may arise in equilibrium. Assume that all buyers hold identical beliefs $\mathbb{E}_{\mathcal{B}}[D]$, all sellers hold identical beliefs $\mathbb{E}_{\mathcal{S}}[D]$ and also assume that $\mathbb{E}_{\mathcal{B}}[D]>\mathbb{E}_{\mathcal{S}}[D]$. In that case:

$$
\operatorname{Cov}_{F}\left(\mathbb{E}_{i}[D], \frac{d X_{1 i}}{d \tau}\right)=\underbrace{\left(\mathbb{E}_{\mathcal{B}}[D]-\mathbb{E}_{\mathcal{S}}[D]\right)}_{>0} \underbrace{\int_{i \in \mathcal{B}} \frac{d X_{1 i}}{d \tau} d F(i)}_{<0}<0,
$$

where lemma 2 allows us to conclude that $\int_{i \in \mathcal{B}} \frac{d X_{1 i}}{d \tau} d F(i)$ is negative.

Main results Propositions 1 and 2 state the main results of the paper.

Proposition 1. (Marginal effect on welfare)

a) The marginal change in social welfare induced by varying the financial transaction tax $\tau$ is given by:

$$
\frac{d V}{d \tau}=\int \underbrace{\lambda_{i} \mathbb{E}\left[U_{i}^{\prime}\left(W_{2 i}\right)\right]}_{\text {Welfare weight }}[[\underbrace{\left(\mathbb{E}[D]-\mathbb{E}_{i}[D]\right)}_{\text {Belief distortion }}+\underbrace{\operatorname{sgn}\left(\Delta X_{1 i}\right) P_{1} \tau}_{\text {Fundamental distortion }}] \frac{d X_{1 i}}{d \tau}-\underbrace{\Delta X_{1 i} \frac{d P_{1}}{d \tau}}_{\text {Terms-of-trade }}] d F(i)
$$

b) Under assumption [NR], the marginal change in social welfare induced by varying the financial transaction tax becomes:

$$
\frac{d V}{d \tau}=\int\left[-\mathbb{E}_{i}[D]+\operatorname{sgn}\left(\Delta X_{1 i}\right) P_{1} \tau\right] \frac{d X_{1 i}}{d \tau} d F(i)
$$

c) Under assumptions [NR] and [OBPS], the marginal change in social welfare induced by introducing a very small tax is strictly positive. Locally around $\tau=0$, there is a first-order gain from reducing trades driven by belief disagreement but there is a second-order loss from reducing fundamental trades, that is:

$$
\left.\frac{d V}{d \tau}\right|_{\tau=0}=-\left.\int \mathbb{E}_{i}[D] \frac{d X_{1 i}}{d \tau}\right|_{\tau=0} d F(i)>0
$$

Proof. See appendix A.

Equation (10) is the weighted sum of the effect of the tax in the indirect utilities of all market participants. The weight given by the planner to the changes in the certainty equivalent of every investor is a combination of the social welfare weight and the (expected) marginal utility. Note that all welfare losses due to belief disagreement ultimately arise because investors hold too much or too little of the risky asset: their risk-return tradeoff is distorted. 
After imposing assumption [NR], two terms drop out from (10). First, terms-of-trade effects cancel out in the aggregate after imposing market clearing. ${ }^{15}$ Intuitively, changes in equilibrium prices are simply a transfer of resources, which are irrelevant for a planner without a redistributive goal in a static setup. Second, the term containing the truth $\mathbb{E}[D]$ also cancels out after imposing market clearing. Because of its conceptual importance, I state this result as a corollary of proposition 1.

Corollary. (Irrelevance of planner's belief) The optimal financial transaction tax does not depend on the true payoff distribution if and only if

$$
\int \lambda_{i} \mathbb{E}\left[U_{i}^{\prime}\left(W_{2 i}\right)\right] \frac{d X_{1 i}}{d \tau} d F(i)=0
$$

This condition holds if assumption [NR] holds.

Although I initially assume that the planner knows the true distribution of returns, as long as (12) holds, the planner could have used any belief to calculate social welfare. ${ }^{16}$ The fact that the planner may not require more information than the investors to evaluate this tax policy is an important takeaway of this paper.

Two characteristics of the economic environment are essential to derive this corollary. First, the risky asset is in fixed supply, which implies that the change in asset holdings must cancel out in the aggregate, that is, $\int \frac{d X_{1 i}}{d \tau} d F(i)=0$. In that case, only relative asset holdings matter for welfare. Second, the planner does not want to redistribute wealth across investors, that is, $\lambda_{i} \mathbb{E}\left[U_{i}^{\prime}\left(W_{2 i}\right)\right]$ is constant. A planner who redistributes resources would overweight the distortions of investors with higher welfare weights. For that, he would need to know the truth.

Brunnermeier, Simsek and Xiong (2012) postulate that a transaction is welfare reducing if under any single belief that is a convex combination of the beliefs held by the investors engaged in trading, the transaction is Pareto dominated by the transaction not taking place. When equation (12) holds, any belief chosen by the planner — not only those in the convex hull of beliefs - would implement the optimal tax, so their criterion would be valid in this context. The worst case scenario criterion proposed by Blume et al. (2013) would also apply here.

The logic behind the local result from proposition 1c is very general. A small transaction tax reduces equally fundamental and non-fundamental trades. However, when there is too much trading, i.e., [OBPS] holds, a marginal reduction in volume is unequivocally beneficial for social welfare.

I now characterize the optimal financial transaction tax. ${ }^{17}$ To ease the exposition, I impose assumption $[\mathrm{NR}]$ from now on and relegate to the appendix the analysis when it does not apply.

\section{Proposition 2. (Optimal financial transaction tax)}

\footnotetext{
${ }^{15}$ Market clearing implies that $\int \frac{d X_{1 i}}{d \tau} d F(i)=0$. Intuitively, changes in equilibrium asset holdings induced by tax changes must cancel in the aggregate. This condition relies on the fixed supply of shares.

${ }^{16}$ This logic is applicable to any environment with belief distortions. First, solve the normative problem assuming that the planner knows the truth. Then exploit the economic structure of the problem to find the conditions under which any planner's belief is valid.

${ }^{17}$ Because the planner's problem is not convex in $\tau$, first order conditions - like equation (13) — are necessary but not sufficient to characterize the optimal $\tau^{*}$ when $V$ is differentiable. I provide conditions for the differentiability of the planner's problem and study non-convexities in appendix B.
} 
a) Under assumption [NR], the optimal financial transaction tax is characterized by:

$$
\tau^{*}=\frac{\int \frac{\mathbb{E}_{i}[D]}{P_{1}} \frac{d X_{1 i}}{d \tau} d F(i)}{\int \operatorname{sgn}\left(\Delta X_{1 i}\right) \frac{d X_{1 i}}{d \tau} d F(i)}
$$

b) Under assumptions [NR] and [OBPS], the optimal financial transaction tax is strictly positive. If assumption [OBPS] does not hold, the optimal policy could be a positive, negative or zero tax.

Proof. See appendix A.

The expression for $\tau^{*}$ optimally trades off the distortion induced by belief disagreement (numerator) against the loss in fundamental trading (denominator). The numerator of (13) can be written as the cross sectional covariance for active investors of expected returns with equilibrium portfolio tax sensitivities — denoted by $\operatorname{Cov}_{\mathcal{F}, \mathcal{T}}[\cdot, \cdot]$ — times the fraction of active investors - denoted by $\zeta(\tau) \equiv \int_{i \in \mathcal{T}} d F(i)>0$ -, that is:

$$
\int \frac{\mathbb{E}_{i}[D]}{P_{1}} \frac{d X_{1 i}}{d \tau} d F(i)=\zeta(\tau) \operatorname{Cov}_{F, \mathcal{T}}\left[\frac{\mathbb{E}_{i}[D]}{P_{1}}, \frac{d X_{1 i}}{d \tau}\right]
$$

When this covariance is negative, $\tau^{*}$ is positive and viceversa. This occurs because the denominator, which can be written as twice the marginal change in trading volume, that is:

$$
\int \operatorname{sgn}\left(\Delta X_{1 i}\right) \frac{d X_{1 i}}{d \tau} d F(i)=2 \int_{i \in \mathcal{B}} \frac{d X_{1 i}}{d \tau} d F(i),
$$

is strictly negative. ${ }^{18}$ Because prices, demand responses and limits of integration are functions of taxes, equation (13) provides an implicit representation for $\tau^{*}$.

An intuitive way to interpret (13) is by rewriting $\tau^{*}$ as half the difference between buyers and sellers (weighted) averages of expected returns, that is:

$$
\tau^{*}=\frac{\Omega_{\mathcal{B}}-\Omega_{\mathcal{S}}}{2}
$$

where $\Omega_{\mathcal{B}}$ and $\Omega_{\mathcal{S}}$ are weighted sums of expected returns of buyers and sellers, respectively, that is:

$$
\Omega_{\mathcal{B}} \equiv \int_{i \in \mathcal{B}} \omega_{i}^{\mathcal{B}} \frac{\mathbb{E}_{i}[D]}{P_{1}} d F(i) \quad \text { and } \quad \Omega_{\mathcal{S}} \equiv \int_{i \in \mathcal{S}} \omega_{i}^{\mathcal{S}} \frac{\mathbb{E}_{i}[D]}{P_{1}} d F(i)
$$

The weights are defined by $\omega_{i}^{\mathcal{B}} \equiv \frac{\frac{d X_{1 i}}{d \tau}}{\int_{i \in \mathcal{B}} \frac{d X_{1 i}}{d \tau} d F(i)}$ for buyers and $\omega_{i}^{\mathcal{S}} \equiv \frac{\frac{d X_{1 i}}{d \tau}}{\int_{i \in \mathcal{S}} \frac{d X_{1 i}}{d \tau} d F(i)}$ for sellers. Note that the weights of active traders $\omega_{i}^{\mathcal{B}}$ and $\omega_{i}^{\mathcal{S}}$ add up to one, and the weights of investors who do not trade are exactly zero. The planner overweights the beliefs of investors with higher sensitivities $\frac{d X_{1 i}}{d \tau}$, because they are the most responsive to tax policy. Intuitively, the magnitude of the optimal tax depends on how much buyers' beliefs differ from sellers' beliefs.

\footnotetext{
${ }^{18}$ Note that $\int \operatorname{sgn}\left(\Delta X_{1 i}\right) \frac{d X_{1 i}}{d \tau} d F(i)$ can be written as an expectation $2 \zeta(\tau) \mathbb{E}_{F, \mathcal{B}}\left[\frac{d X_{1 i}}{d \tau}\right]$. Hence, the fraction of active investors $\zeta(\tau)$ effectively cancels out of the formula for $\tau^{*}$.
} 
Sufficient statistics The joint distribution of investors' equilibrium portfolio tax sensitivities and investors' beliefs directly determine the optimal tax, independently of the rest of the structure of the model. The cross-sectional covariance between investor's beliefs and equilibrium portfolio tax sensitivities becomes the relevant sufficient statistic to determine the optimal tax. ${ }^{19}$

When all portfolio tax sensitivities are identical in magnitude, that is, $\left|\frac{d X_{1 i}}{d \tau}\right|$ is constant, $\omega_{i}^{\mathcal{B}}=\omega_{i}^{\mathcal{S}}=1$ for all investors. In that case, $\tau^{*}$ only depends on the difference in beliefs between buyers and sellers and the distribution of beliefs becomes the single sufficient statistic for the determination of the optimal tax. Portfolio tax sensitivities drop out of the optimal tax formula. This is an important benchmark for the actual implementation of the tax, since it has minimal informational requirements for the planner. Appendix B presents a parametric example when beliefs are normally distributed. In that case, the optimal tax is an increasing monotonic function of the variance of the distribution of beliefs.

\subsection{Remarks}

I summarize several implications of propositions 1 and 2 in a series of remarks.

Remark 1. The total amount of fundamental trading only determines the optimal tax through the composition of the marginal investors.

It may be natural to think that the optimal tax formula should feature a tradeoff between measures of fundamental trading - like the absolute value of the covariance terms $\operatorname{Cov}\left[E_{2 i}, D\right]$ or the dispersion among investors risk aversion coefficients - versus measures of non-fundamental trading. This logic is flawed. Intuitively, the optimal tax only depends on the marginal distortions on trading, i.e. $\frac{d X_{1 i}}{d \tau}$, but not on the total amount of trading.

However, fundamental trading does affect the optimal tax through the limits of integration — which determine, on the extensive margin, who are the marginal investors in equilibrium for every value $\tau$ - and potentially through portfolio tax sensitivities. ${ }^{20}$ In other words, a marginal change in beliefs $\mathbb{E}_{i}[D]$ for active investors always changes the value of the optimal tax. However, a marginal change in measures of fundamental trading only modifies the optimal tax by varying the composition of investors who actively trade or not for a given $\tau$.

Remark 2. Investors who decide not to trade are inframarginal for the determination of the optimal tax.

Only the beliefs and portfolio tax sensitivities of the marginal investors matter for the determination of the optimal tax in equilibrium. Intuitively, there is an envelope condition which also acts at the margin of trading versus not trading. Those investors who optimally decide not to trade become inframarginal from an optimal taxation perspective. Hence, the optimal tax accounts for the fact that some trades cease to occur when transaction taxes are imposed. In appendix B, I analyze the tight relation between extensive margin changes and non-convexities in the planner's problem.

\footnotetext{
${ }^{19}$ The sufficient statistic logic is similar to the one behind the CAPM, in which the beta of an asset becomes sufficient to determine expected returns. It is also similar to the logic behind consumption based asset pricing models, in which the consumption process, independently of how it is generated, is sufficient to determine asset prices and expected returns.

${ }^{20}$ Because of the CARA-Normal assumption, $\frac{d X_{1 i}}{d \tau}$ does not depend directly on $\operatorname{Cov}\left[E_{2 i}, D\right]$, but this may not be the case in a more general framework.
} 
This remark implies that quadratic taxes - which are often used as a tractable approximation for linear taxes - are not a good approximation for linear taxes, because they imply that all investors are marginal for all values of $\tau$.

Remark 3. The dispersion of beliefs across investors, regardless of whether they are right or wrong on average, is what determines the optimal tax.

For instance, if all investors agree about the expected payoff the risky asset, that is, $\mathbb{E}_{i}[D]$ is constant, the optimal tax is $\tau^{*}=0$, independently of whether they are right or wrong. This situation occurs naturally when all investors hold correct beliefs about the distribution of $D-$ this is the standard common prior result. However, if all investors are wrong, the optimal tax is also zero. Only belief dispersion matters because the equilibrium portfolio allocation, which is what ultimately matters for welfare, only depends on the relative dispersion on beliefs.

Remark 4. The optimal policy can be a subsidy, that is, $\tau^{*}<0$.

Although the exposition focuses on the $\tau^{*}>0$ case - the relevant case under [OBPS] -, if many optimists happen to be sellers of the risky asset in the laissez-faire equilibrium, instead of buyers, the optimal policy may be a subsidy. Intuitively, if investors trade too little, it is optimal for the planner to encourage them to trade more with a subsidy. In appendix B, I show how a subsidy may emerge as the optimal policy when [OBPS] does not hold.

Remark 5. Changes in trading volume, and not price changes, are the welfare relevant sufficient statistics.

Although many informal arguments surrounding transaction taxes focus on their effects on prices, this paper shows that changes in social welfare are driven exclusively by changes in volume. From a welfare standpoint, the correct question is not whether prices are right, but whether every investor is holding the right amount of risk.

Price changes play a role redistributing wealth, which a planner without a redistributive goal ignores when setting policies. ${ }^{21}$ In sections 6 and 7, I show how this logic extends to dynamic environments and production economies.

Remark 6. The restriction to a single linear tax implies that portfolio tax sensitivities $\frac{d X_{1 i}}{d \tau}$ determine the weights given to individual beliefs in the optimal tax formula.

Classic Pigovian logic suggests that an optimal corrective tax only depends on the magnitude of the distortion but not on elasticities. However, the optimal tax in (13) depends on portfolio tax sensitivities. This occurs because the planner uses a second-best policy instrument. Intuitively, the planer would like to eliminate every belief distortion individually. However, because he has a single policy instrument,

\footnotetext{
${ }^{21}$ It may seem that the planner only seeks to improve the welfare of investors with incorrect beliefs. That is not necessarily the case, because of general equilibrium effects. It is true that tax policy can only increase aggregate social welfare if some investors hold distorted beliefs. However, which particular investors benefit or lose from the tax policy depends on how prices adjust in general equilibrium. Nonetheless, in this model, these price adjustments must add up to zero in dollar terms. There is scope to explore whether, in a richer model, investors with incorrect beliefs can have a disproportionally large effect in other market participants - for instance, introducing some form of strategic complementarity, following the logic in Haltiwanger and Waldman $(1985,1989)$.
} 
it turns out to be optimal to weight distortions according to demand sensitivities. The planner weights more the distortions of the most responsive investors.

The presence of demand sensitivities in optimal corrective tax formulas goes back to Diamond (1973), who analyzes corrective taxation with restricted policy instruments in a model of consumption externalities. My results are directly related to the separable case analyzed in that paper. ${ }^{22}$

\subsection{Harberger revisited}

The results derived so far rely on the assumption that the planner maximizes welfare using a single belief. However, it is straightforward to quantify the welfare loss induced by a tax increase assuming: a) that all investors hold correct beliefs or $b$ ) that the planner assess social welfare respecting individual beliefs. Under either of these assumptions, all trades are regarded as fundamental, so any tax induces a welfare loss. I derive a result analogous to Harberger (1964).

\section{Proposition 3. (Harberger (1964) revisited)}

a) When investors hold correct beliefs or the planner respects individual beliefs when calculating social welfare, the marginal welfare loss generated by increasing the transaction tax at a level $\tilde{\tau}$, expressed as a money-metric (in dollars) at $t=1$, is given by:

$$
\left.\int \frac{d \hat{V}_{i}}{d \tau}\right|_{\tau=\tilde{\tau}} d F(i)=\left.2 \tilde{\tau} P_{1} \int_{i \in \mathcal{B}} \frac{d X_{1 i}}{d \tau}\right|_{\tau=\tilde{\tau}} d F(i) \leq 0
$$

Where $i \in \mathcal{B}$ denotes that the integration is made only over the set of buyers and $\hat{V}_{i}$ denotes investors' certainty equivalents.

b) The marginal welfare loss of a small tax change around $\tau=0$ can be approximated, using a second order Taylor expansion, by:

$$
d V=\left.\left.\int d \hat{V}_{i}\right|_{\tau=0} d F(i) \approx \tau^{2} P_{1} \int_{i \in \mathcal{B}} \frac{d X_{1 i}}{d \tau}\right|_{\tau=0} d F(i)
$$

Proof. See appendix A.

This proposition provides a direct welfare measure as a function of observables for any tax intervention. In particular, given the money-metric correction, investors in this economy are willing to pay $\mathcal{L}(\tau)$ dollars to prevent a change in the tax rate. This happens to correspond in this model to the total revenue raised. Note that when $\tau=0$, a marginal increase in the tax only has second-order effects in welfare.

Equation (15) resembles the classic Harberger (1964) result about welfare losses in the context of commodity taxation. ${ }^{23}$ Taxing a commodity distorts the amount consumed of a given good, reducing

\footnotetext{
${ }^{22}$ Most of the results in Diamond (1973) focus on how the optimal corrective tax should account for demand inter-linkages. Those issues never arise in this paper, because the portfolio allocation of an investor $i$ does not depend directly (only through prices) on the belief distortions of other investors.

${ }^{23}$ Although this result is intuitive, to my knowledge, it had not been derived before in the context of a portfolio choice problem. See Auerbach and Hines Jr (2002) for a comprehensive analysis of tax efficiency results and Sandmo (1985) for a survey of results on how taxation affects portfolio allocations.
} 
welfare. Taxing financial transactions distorts portfolio allocations, inducing investors to hold more or less risk than they should, also reducing welfare. The idea that the distortion created by a tax (approximately) grows with its square is also associated to Harberger (1964) - equation (16) presents the equivalent result in the context of this model.

As expected, the change in dollar volume induced by the tax change, given by $P_{1} \int_{i \in \mathcal{B}} \frac{d X_{1 i}}{d \tau} d F(i)$, is the correct measure for welfare losses. In this static context, under the money-metric criterion, price changes are mere transfers. The welfare loss is twice the size of the tax, because the portfolio holdings of both buyers and sellers are distorted. I show in page 41 that, in a dynamic model, the welfare loss doubles when investors alternate between buying and selling every period, that is, it corresponds to four times the size of the tax.

Finally, observe that the assumptions used to calculate social welfare can dramatically change the interpretation of a reduction in volume following a tax increase. If all trading is fundamental, a reduction in volume only generates welfare losses. If there is some non-fundamental trading, part of that volume reduction has positive effects on social welfare. Therefore, equation (15) can be seen as an upper bound on the size of the welfare losses.

\section{Numerical Simulations}

To provide further intuition, I illustrate the previous results with several numerical examples. In all examples, I impose the following restrictions, summarized in table 1. First, all investors have the same absolute risk aversion coefficient $A_{i}=1$ and the same initial holdings of the risky asset $X_{0 i}=Q=1$. Therefore, hedging needs become the only source of fundamental trading. Second, the planner uses welfare weights $\lambda_{i}=1$ and implements transfers so that $\mathbb{E}\left[U_{i}^{\prime}\left(W_{2 i}\right)\right]$ is constant for all investors for every value of $\tau$. Under these restrictions, assumption [NR] holds and welfare corresponds directly to investors' certainty equivalents.

$$
\begin{array}{|l|l|l|}
\hline \lambda_{i}=1, \forall i & \mathbb{E}\left[U_{i}^{\prime}\left(W_{2 i}\right)\right] \text { constant, } \forall i & X_{0 i}=Q=1, \forall i \\
\hline
\end{array}
$$

Table 1: Restrictions across all examples

Although I have chosen reasonable parameters, a serious calibration is beyond the scope of this paper. All figures are in the appendix.

Example 1. (Only optimists and pessimists/No fundamental trading) There are two groups of investors. A fraction $\pi_{H}$ of them is of type $i=H$ and the remainder fraction $\pi_{L}=1-\pi_{H}$ is of type $i=L$, where $H$ and $L$ correspond to high (optimists) and low (pessimists) valuations for the risky asset. The rest of parameters are in Table 2 .

\begin{tabular}{|c|c|c|c|}
\hline$\pi_{H}=0.5$ & $\mathbb{E}_{H}[D]=106$ & $\operatorname{Cov}\left[E_{2 H}, D\right]=0$ & $\mathbb{E}[D]=100$ \\
\hline$\pi_{L}=0.5$ & $\mathbb{E}_{L}[D]=96$ & $\operatorname{Cov}\left[E_{2 L}, D\right]=0$ & $\mathbb{V a r}[D]=16$ \\
\hline
\end{tabular}

Table 2: Parameters Example 1 - Figure 4 
Under this parametrization there is no fundamental trading. The payoff mistake made by optimists corresponds to 1.5 standard deviations and the one made by pessimists to a single standard deviation. Figure 4 shows welfare relative to the $\tau=0$ case, equilibrium allocations, price and volume for different values of $\tau$. The optimal tax is $\tau^{*}=5.88 \%$ and the welfare gain at the optimum is $0.86 \%{ }^{24}$

\section{FIGURE 4 ABOUT HERE — SEE PAGE 55}

Because buyers and sellers are symmetric in all dimensions but beliefs, the equilibrium price is constant for any level of the tax, eliminating terms-of-trade effects. Volume decreases in the level of the tax, as expected. When $\tau>5.88 \%$, trading stops and all investors simply keep their initial holdings of the risky asset. It is not a coincidence that the optimal tax is the smallest one which eliminates trade. This example illustrates the following important insight.

Remark. If all trading is driven by disagreement, the optimal tax completely shuts down trading.

The welfare of the optimists is monotonically increasing in the tax. However, the welfare of pessimists peaks at a tax rate $\tau=4.71 \%$ and then it decreases. Intuitively, pessimists would be happier with a smaller tax, because they are making a smaller error.

Starting from this parametrization, an increase in the amount of fundamental trading would not change the optimal tax. Investors would start to trade, but the optimal tax would not change.

\section{Example 2. (Optimists, pessimists and correct investors)}

In addition to optimists and pessimists, now there is group of correct investors in the economy who trade for risk-sharing purposes. A proportion $\pi_{A}$ of investors are correct and have an endowment that comoves positively with the risky asset. The rest of the correct investors, in proportion $\pi_{B}$, have a negatively correlated endowment. Optimists and pessimists have proportions $\pi_{H}$ and $\pi_{L}=$ $1-\pi_{H}-\pi_{A}-\pi_{B}$ respectively. Investors with correct beliefs, can be considered as arbitrageurs as described, for instance, in Shleifer (2000). Table 3 describes the parametrization.

\begin{tabular}{|c|c|c|c|}
\hline$\pi_{H}=0.25$ & $\mathbb{E}_{H}[D]=106$ & $\operatorname{Cov}\left[E_{2 H}, D\right]=0$ & $\mathbb{E}[D]=100$ \\
\hline$\pi_{L}=0.25$ & $\mathbb{E}_{L}[D]=96$ & $\operatorname{Cov}\left[E_{2 L}, D\right]=0$ & $\mathbb{V a r}[D]=16$ \\
\hline$\pi_{A}=0.25$ & $\mathbb{E}_{A}[D]=100$ & $\operatorname{Cov}\left[E_{2 A}, D\right]=12$ & \\
\hline$\pi_{B}=0.25$ & $\mathbb{E}_{B}[D]=100$ & $\operatorname{Cov}\left[E_{2 B}, D\right]=-5$ & \\
\hline
\end{tabular}

Table 3: Parameters Example 2 - Figure 5

These parameters imply that $40.91 \%$ of the trading volume at $t=0$ is fundamental and the remaining $59.09 \%$ is due to disagreement. ${ }^{25}$ The optimal tax is $\tau^{*}=2.98 \%$, approximately half than in the previous example and the welfare gain at the optimum is $0.21 \%$.

\footnotetext{
${ }^{24}$ This is a sizable gain when compared to the classic Lucas (1987) calculation. This is not surprising, given that investors are not at their optimal portfolios from the planner's perspective.

${ }^{25}$ To find these values, I divide equilibrium volume when investors hold heterogeneous beliefs over equilibrium value assuming that all investors hold the same belief.
} 


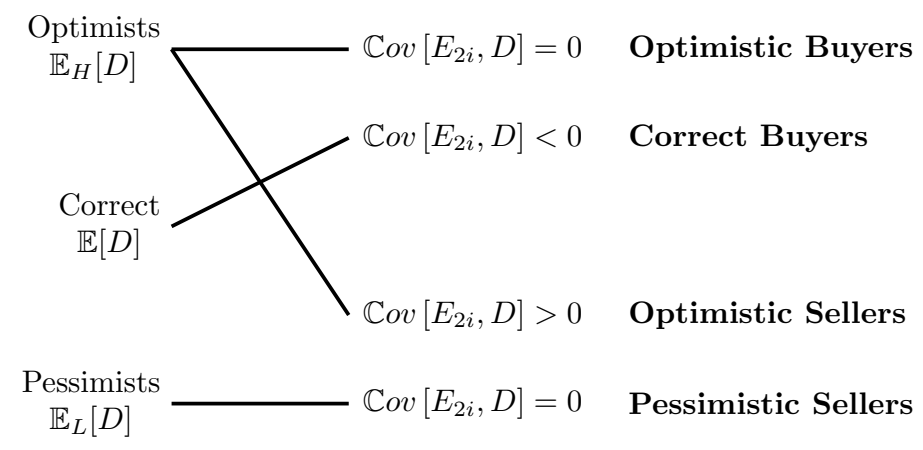

Figure 2: Pattern of beliefs and hedging needs

\section{FIGURE 5 ABOUT HERE — SEE PAGE 56}

Note that now only $50 \%$ of the investors hold distorted beliefs, which reduces the magnitude of the optimal tax to roughly half of the value in example 1 . The equilibrium price is constant for most of the levels of $\tau$, although it drifts downward for the values of $\tau$ in which investor $L$ (a seller) stops trading but before investor $B$ (a buyer) stops trading.

\section{Example 3. (Pessimists buyers)}

This example shows how a tax can create, even locally, a first-order loss for some investors. This is the effect that, when sufficiently large, can support a subsidy in equilibrium.

There is a fraction $\pi_{H A}$ optimists who buy the asset with no hedging motive and a fraction $\pi_{H B}$ who happen to sell the risky asset for risk sharing reasons. A fraction $\pi_{C}$ of correct investors must buy the risky asset for risk sharing reasons and the remainder fraction $\pi_{L}$ of investors are pessimists with no hedging motive. Table 4 describes the parametrization, graphically represented in figure 2.

\begin{tabular}{|c|c|c|c|}
\hline$\pi_{H A}=0.3$ & $\mathbb{E}_{H A}[D]=106$ & $\operatorname{Cov}\left[E_{2 H A}, D\right]=0$ & $\mathbb{E}[D]=100$ \\
\hline$\pi_{H B}=0.2$ & $\mathbb{E}_{H B}[D]=106$ & $\operatorname{Cov}\left[E_{2 H B}, D\right]=7$ & $\mathbb{V a r}[D]=16$ \\
\hline$\pi_{C}=0.2$ & $\mathbb{E}_{C}[D]=100$ & $\operatorname{Cov}\left[E_{2 C}, D\right]=-8$ & \\
\hline$\pi_{L}=0.3$ & $\mathbb{E}_{L}[D]=96$ & $\operatorname{Cov}\left[E_{2 L}, D\right]=0$ & \\
\hline
\end{tabular}

Table 4: Parameters Example 3 - Figure 6

These parameters imply that roughly $65 \%$ of the trading volume at $\tau=0$ is fundamental and the remaining $35 \%$ is due to disagreement. The optimal tax is $\tau^{*}=2.01 \%$ and the welfare gain at the optimum is $0.11 \%$.

Around $\tau=0$,the slope of the indirect utility for a given investor can be positive, negative or zero, even when prices are locally constant. In particular, investors $H A$ and $L$ have positive slopes, which implies that they experience a first-order gain from taxation. The slope of investor $C$, who only trades for fundamental reasons, is flat, since he only experiences a second-order loss. The slope of investor $H B$ is strictly negative; any tax induces a first-order loss for him, since it moves him further away from his optimum, starting from a point at which he was not already optimizing from the planner's perspective. 


\section{Static Extensions}

Within the CARA-Normal static framework, I now analyze, one at a time, several extensions of the baseline model. To ease the exposition, I only describe the essential differences of every extension with respect to the baseline model and use assumption [NR] throughout. I also omit definitions of equilibrium — they are all standard — and most regularity conditions.

\subsection{Pre-existing trading costs}

Because actual investors face trading costs even when there are no taxes, it is natural to think that the results derived around the point $\tau=0$ are never valid. This logic is flawed. Propositions 1 and 2 still apply as long as transaction costs are a mere compensation for the use of economic resources.

Assumptions Investors now face transaction costs, regardless of the value of $\tau$. These represent costs associated with trading, like brokerage commissions, exchange fees or bookkeeping costs. Investors must pay a quadratic cost, parametrized by $\alpha$, a linear cost $\eta$ on the number of shares traded and a linear $\operatorname{cost} \psi$ on the dollar volume of the transaction. ${ }^{26}$ These trading costs are paid to a new group of agents (intermediaries) which facilitate the process of trading. Crucially, I assume that intermediaries make zero profits in equilibrium. Hence, wealth at $t=2$ for an investor $i$ is now given by:

$$
W_{2 i}=E_{2 i}+X_{1 i} D+\left(X_{0 i} P_{1}-X_{1 i} P_{1}-\left|\Delta X_{1 i}\right|\left|P_{1}\right|(\tau+\psi)-\eta\left|\Delta X_{1 i}\right|-\frac{\alpha}{2}\left(\Delta X_{1 i}\right)^{2}+T_{1 i}\right)
$$

The transfer exclusively rebates to the investor the amount paid as a tax, that is, $T_{1 i}=\tau\left|P_{1}\right|\left|\Delta X_{1 i}\right|$.

Results The demand for the risky asset takes a similar form as in the baseline model, featuring also an inaction region, now determined jointly by the trading costs and the transaction tax. The optimal portfolio given prices can be compactly written in the trade region as:

$$
X_{1 i}=\frac{\mathbb{E}_{i}[D]-A_{i} \operatorname{Cov}\left[E_{2 i}, D\right]-P_{1}\left(1+\operatorname{sgn}\left(\Delta X_{1 i}\right)(\tau+\psi)\right)-\operatorname{sgn}\left(\Delta X_{1 i}\right) \eta+\alpha X_{0 i}}{A_{i} \mathbb{V} \operatorname{Var}[D]+\alpha}
$$

All three types of trading costs - quadratic, linear in shares and linear in dollar value — shift investors portfolio towards their initial positions. The equilibrium price is a slightly modified version of (7) - see appendix A.

When calculating welfare, the planner takes into account that investors must incur in these costs when trading - this is the natural constrained efficient benchmark. The optimal tax formula remains unchanged when investors face transaction costs, as long as these trading costs represent exclusively a compensation for the use of economic resources.

\footnotetext{
${ }^{26}$ I do not analyze fixed costs for technical reasons. Any fixed cost would introduce a non-convexity, which may substantially change the equilibrium characterization. See Gârleanu, Panageas and Yu (2013) for recent work with non-convexities in a related environment.
} 
Proposition 4. (Pre-existing trading costs) Under assumption [NR], when investors face trading costs as specified in (17), the expressions for $\frac{d V}{d \tau}$ and $\tau^{*}$ are identical to those in propositions 1 and 2.

Proof. See appendix A.

The intuition behind proposition 4 is similar to the baseline case. An envelope condition eliminates any term regarding transaction costs from $\frac{d V}{d \tau}$, because the planner must also face such costs, so the optimal tax looks identical to the one in the baseline model. This relies on the assumption that the economic profit made by the intermediaries who receive the transaction costs is zero - there cannot be economic rents.

Does proposition 4 imply that if there were two authorities with taxation power, they would both impose the same $\tau^{*}$ twice? Of course not. Assume for simplicity that they set taxes sequentially. The first authority would set the optimal tax according to proposition 2 . The second authority, internalizing that the pre-existing tax is a mere transfer and does not correspond to a compensation for costs of trading, would set a zero tax. Alternatively, $\tau^{*}$ would characterize the sum of both taxes.

This result has further implications. First, although the optimal tax formula does not vary, an economy with transaction costs has less trade in equilibrium than one without transaction costs. Depending on whether this reduction in trading is of the fundamental type or not, the optimal tax may be larger or smaller. Transaction costs only affect the optimal tax through extensive margin changes; see the example below. Second, the mere existence of transaction costs does not provide a new rationale for further discouraging non-fundamental trading. Welfare losses only arise through wedges derived from portfolio distortions. Third, if transactions costs, that is, $\psi, \eta$ and $\alpha$, were endogenously functions of $\tau$, the planner would have to take into account those effects when solving for optimal taxes. ${ }^{27}$ However, if those are determined efficiently, the envelope theorem would still apply, leaving proposition 4 unchanged - portfolio tax sensitivities, whose value may now depend on how transaction costs are determined, would remain as the key sufficient statistic.

Numerical Example To build intuition, I analyze the following numerical example.

\section{Example 4. (Pre-existing linear costs)}

Without transaction costs, the environment is identical to the one in example 2. For simplicity, I set $\eta=0$ and $\alpha=0$, and compare how the equilibrium and the optimal tax changes when the linear dollar transaction cost $\psi$ varies. Figure 7 compares trading volume, allocation, prices and welfare (relative to the zero tax case) for $\psi=0$ and $\psi=2 \%$.

\section{FIGURE 7 ABOUT HERE — SEE PAGE 58}

\footnotetext{
${ }^{27}$ The Walrasian approach of this paper does not capture market microstructure effects as, for instance, Glosten and Milgrom (1985) or Duffie, Gârleanu and Pedersen (2005). There is scope for understanding how transaction taxes affect market making and liquidity provision in greater detail, introducing, for instance, imperfectly competitive agents, search or network frictions. The argument made in this paper should nonetheless remain robust to different market microstructures.
} 
In general, when $\psi$ is very small, the optimal tax does not change. However, for larger values of $\psi$, like $2 \%$, changes on the extensive margin directly affect the optimal tax. When $\psi$ is larger, volume is always lower in equilibrium and prices can move in any direction - the logic from lemmas 1 and 2 applies directly here. Welfare is lower in absolute terms - this is intuitive, since now resources are spent in any transaction - but in general the optimal tax with transaction cots can be higher or lower than without transaction cots. Given this particular parametrization, the optimal $\operatorname{tax} \tau^{*}$ when $\psi=2 \%$ is lower than when there are no transaction costs. This occurs because investor $L$, who was exclusively trading for non-fundamental reasons, stops trading when transaction costs are present. Hence, increasing $\tau$ is socially less beneficial, since it deters fewer non-fundamental trades at the margin.

\subsection{Multiple risky assets}

Assumptions The results of the baseline model extend naturally to an environment with multiple assets. Now there are $J$ risky assets in fixed supply, in addition to the riskless asset. The $J \times 1$ vectors of total shares, equilibrium prices and dividend payments are respectively denoted by $\mathbf{q}, \mathbf{p}$ and $\mathbf{d} .{ }^{28}$ Every purchase or sale of a risky asset faces an identical linear transaction tax $\tau$. This is a further restriction on the planner's problem, since belief distortions can vary across different assets, but the tax must be constant. Allowing for different taxes for different (groups of) assets is nonetheless straightforward, following the logic of subsection 5.5 .

The objective distribution of the dividends $\mathbf{d}$ paid by the risky assets is a multivariate normal with mean $\mathbb{E}[\mathbf{d}]$ and variance-covariance matrix $\mathbb{V a r}[\mathbf{d}]$. All investors agree about the variance, but an investor $i$ believes that the mean of $\mathbf{d}$ is $\mathbb{E}_{i}[\mathbf{d}]$. We can thus write:

$$
\mathbf{d} \sim N(\mathbb{E}[\mathbf{d}], \mathbb{V a r}[\mathbf{d}]) \quad \text { and } \quad \mathbf{d} \sim_{i} N\left(\mathbb{E}_{i}[\mathbf{d}], \mathbb{V a r}[\mathbf{d}]\right)
$$

Risk aversion $A_{i}$, and the vectors of initial asset holdings $\mathbf{x}_{0 i}$, hedging needs $\operatorname{Cov}\left[E_{2 i}\right.$, $\left.\mathbf{d}\right]$ and beliefs $\mathbb{E}_{i}[\mathbf{d}]$ are arbitrary across the distribution of investors. The wealth at $t=2$ of an investor $i$ is thus given by:

$$
W_{2 i}=E_{2 i}+\mathbf{x}_{1 i}^{\prime} \mathbf{d}+\left(\mathbf{x}_{0 i}^{\prime} \mathbf{p}-\mathbf{x}_{1 i}^{\prime} \mathbf{p}-\left|\mathbf{x}_{1 i}^{\prime}-\mathbf{x}_{0 i}^{\prime}\right| \mathbf{p} \tau+T_{1 i}\right)
$$

Results The first order condition (18) characterizes the solution of this problem for the set of assets traded:

$$
\mathbf{x}_{1 i}=\left(A_{i} \mathbb{V} \operatorname{ar}[\mathbf{d}]\right)^{-1}\left(\mathbb{E}_{i}[\mathbf{d}]-A_{i} \operatorname{Cov}\left[E_{2 i}, \mathbf{d}\right]-\mathbf{p}-\hat{\mathbf{p}}_{i} \tau\right),
$$

where $\hat{\mathbf{p}}_{i}$ is a $J \times 1$ vector where row $j$ is given by $\operatorname{sgn}\left(\Delta X_{1 i j}\right) p_{j}$ and $p_{j}$ denotes the price of asset $j$. If an asset $j$ is not traded by an investors $i$, then $X_{1 i j}=X_{0 i j}$. If asset returns are independent, the portfolio allocation to every asset can be determined in isolation.

Equilibrium prices are a natural extension of the baseline model — see appendix A.

\footnotetext{
${ }^{28}$ I use bold lower-case letters to denote vectors but, for consistency, I keep the upper-case notation for holdings of a single asset.
} 


\section{Proposition 5. (Multiple risky assets)}

a) Under assumption [NR], the marginal change in social welfare from varying the financial transaction tax when investors can trade J risky assets is given by:

$$
\frac{d V}{d \tau}=\int\left(\mathbb{E}[\mathbf{d}]-\mathbb{E}_{i}[\mathbf{d}]+\hat{\mathbf{p}}_{i} \tau\right)^{\prime} \frac{d \mathbf{x}_{1 i}}{d \tau} d F(i)
$$

b) Under assumption [NR], the optimal tax when investors can trade J risky assets is given by:

$$
\tau^{*}=\sum_{j=1}^{J} \omega_{j} \tau_{j}^{*}
$$

With weights $\omega_{j}$ and single-asset taxes $\tau_{j}^{*}$ given respectively by $\omega_{j} \equiv \frac{p_{j} \int \operatorname{sgn}\left(\Delta X_{1 i j}\right) \frac{d X_{1 i j}}{d \tau} d F(i)}{\sum_{j=1}^{J} p_{j} \int \operatorname{sgn}\left(\Delta X_{1 i j}\right) \frac{d X_{1 i j}}{d \tau} d F(i)}$ and $\tau_{j}^{*} \equiv$ $\frac{\int \frac{\mathbb{E}_{i}\left[D_{j}\right]}{p_{j}} \frac{d X_{1 i j}}{d \tau} d F(i)}{\int \operatorname{sgn}\left(\Delta X_{1 i j}\right) \frac{d X_{1 i j}}{d \tau} d F(i)}$.

Proof. See appendix A.

The formula for $\tau_{j}^{*}$ is identical to the one in an economy with a single risky asset. The optimal tax in a model with $J$ risky assets is simply a weighted average of all $\tau_{j}^{*}$. The weights are determined by the relative marginal changes in (dollar) volume. Those assets whose volume responds more aggressively to tax changes carry higher weights when determining the optimal tax and viceversa.

\subsection{Portfolio constraints: short-sale and borrowing constraints}

Assumptions Although participants in financial markets face short-sale and borrowing constraints, investors in the baseline model face no restrictions when choosing portfolios. I now introduce trading constraints into the model as a pair of functions $\bar{g}_{i}(\cdot)$ and $\underline{g}_{i}(\cdot)$ for every investor $i$, which can depend on equilibrium prices, ${ }^{29}$ such that:

$$
\underline{g}_{i}\left(P_{1}\right) \leq X_{1 i} \leq \bar{g}_{i}\left(P_{1}\right)
$$

Both short-sale constraints and borrowing constraints are special cases of (20). Short-sale constraints are in general price independent and can be written as $X_{1 i} \geq 0$. Borrowing constraints can be modeled by choosing $\bar{g}_{i}\left(P_{1}\right)$ appropriately, such that $X_{1 i} \leq \bar{g}_{i}\left(P_{1}\right)$. Intuitively, an investor who wants to sufficiently increase his holdings of the risky asset must rely on borrowing. Hence, a borrowing limit is equivalent to an upper bound constraining the amount held of the risky asset.

Results The optimal portfolio is identical to the one in the baseline model, unless a constraint binds. In that case, $X_{1 i}$ equals the trading limit. The equilibrium price is a slightly modified version of (7) see appendix A.

\section{Proposition 6. (Trading constraints)}

\footnotetext{
${ }^{29} \mathrm{I}$ assume that investor's choice sets remain convex after imposing (20). By $g_{i}(\cdot)$, I denote either $\bar{g}_{i}(\cdot)$ or $\underline{g}_{i}(\cdot)$.
} 
a) Under assumption [NR], the marginal change in social welfare from varying the financial transaction tax when investors face trading constraints is given by:

$$
\frac{d V}{d \tau}=\int\left[-\mathbb{E}_{i}[D]+\operatorname{sgn}\left(\Delta X_{1 i}\right) P_{1} \tau\right] \frac{d X_{1 i}}{d \tau} d F(i)+\int_{i=\mathcal{C}} A_{i} \mathbb{V} \operatorname{ar}[D]\left(\hat{X}_{1 i}-g_{i}\left(P_{1}\right)\right) \frac{d X_{1 i}}{d \tau} d F(i),
$$

where $i=\mathcal{C}$ denotes the set of investors with binding trading constraints and $\tilde{X}_{1 i}$ denotes the optimal unconstrained portfolio holding for a constrained investor - see appendix A.

b) Under assumption [NR], the optimal tax when investors face trading constraints is given by:

$$
\tau^{*}=\frac{\int \mathbb{E}_{i}[D] \frac{d X_{1 i}}{d \tau} d F(i)-\int_{i=\mathcal{C}} A_{i} \mathbb{V} \operatorname{ar}[D]\left(\hat{X}_{1 i}-g_{i}\left(P_{1}\right)\right) g_{i}^{\prime}\left(P_{1}\right) \frac{d P_{1}}{d \tau} d F(i)}{P_{1} \int \operatorname{sgn}\left(\Delta X_{1 i}\right) \frac{d X_{1 i}}{d \tau} d F(i)},
$$

where I have used the fact that $\frac{d X_{1 i}}{d \tau}=g_{i}^{\prime}\left(P_{1}\right) \frac{d P_{1}}{d \tau}$ for constrained investors.

Proof. See appendix A.

When trading constraints do not depend on prices that is, $g_{i}^{\prime}\left(P_{1}\right)=0$, the optimal tax formula is identical to the one of the baseline model. In those cases, changes in taxes do not modify the portfolio allocation of constrained investors, leaving their welfare unchanged, i.e., for those investors $\frac{d X_{1 i}}{d \tau}=0$. Intuitively, investors with price independent trading constraints are inframarginal for price determination.

When trading constraints depend on prices, the optimal policy takes these effects into account. A marginal tax change modifies asset prices and consequently portfolio allocations for constrained agents; this portfolio change has a first-order effect on welfare. The size of the correction has three determinants. First, it depends on how far the actual portfolio allocation is from the unconstrained portfolio allocation, given by how much the constrained allocation $g_{i}\left(P_{1}\right)$ differs from the optimal unconstrained allocation $\hat{X}_{1 i}$. Second, it depends on how sensitive the equilibrium restriction is with respect to asset prices this is captured by $g_{i}^{\prime}\left(P_{1}\right)$. Third, it depends on how equilibrium prices react to tax changes $\frac{d P_{1}}{d \tau}$. If prices remain constant after varying $\tau$, that is $\frac{d P_{1}}{d \tau}=0$, the optimal tax formula does not change. ${ }^{30}$

\subsection{Disagreement about second moments}

Assumptions In the baseline model, investors only disagree about the expected value of the payoff of the risky asset. I now assume that investors also hold distorted beliefs about their hedging needs $\operatorname{Cov}_{i}\left[E_{2 i}, D\right]$ and about the variance of the payoff of the risky asset $\operatorname{Var}_{i}[D]$.

Results The optimality condition presented in (5) applies directly, after using the individual beliefs of each investor. Hedging needs enter additively, but perceived individual variances modify the sensitivity of portfolio demands with respect to the baseline case.

\footnotetext{
${ }^{30}$ This result is related to the literature on pecuniary externalities through collateral constraints. See, for instance, Jeanne and Korinek (2010), Bianchi (2011), Stein (2012) or Davila (2011). The externalities discussed in those papers interact here with the determination of transaction taxes.
} 
Market clearing determines the equilibrium price, given now by:

$$
P_{1}=\frac{\int_{i \in \mathcal{T}}\left(\frac{\mathbb{E}_{i}[D]}{\mathcal{A} \mathcal{V}_{i}}-A V\left(\beta_{i i}+X_{0 i}\right)\right) d F(i)}{1+\tau \int_{i \in \mathcal{T}} \frac{\operatorname{sgn}\left(\Delta X_{1 i}\right)}{\mathcal{A} \mathcal{V}_{i}} d F(i)}
$$

where $A V \equiv\left(\int_{i \in \mathcal{T}} \frac{1}{A_{i} \mathbb{V a r} r_{i}[D]} d F(i)\right)^{-1}$ is the harmonic mean of risk aversion coefficients and perceived variances for active investors and $\mathcal{A} \mathcal{V}_{i} \equiv \frac{A_{i} \mathbb{V} a r_{i}[D]}{A V}$ is the quotient between investor $i$ risk aversion times perceived variance and the harmonic mean. I define the regression coefficient (beta) of individual endowments $E_{2 i}$ on payoffs $D$ perceived by investors by $\beta_{i i}=\frac{\operatorname{Cov}_{i}\left[E_{2 i}, D\right]}{\mathbb{V} a r_{i}[D]}$. Again, $\mathcal{T}$ denotes the set of active investors.

\section{Proposition 7. (Disagreement about second moments)}

a) Under assumption [NR], the marginal change in social welfare from varying the financial transaction tax when investors disagree about second moments is given by:

$$
\frac{d V}{d \tau}=\int\left[\left(-r_{i} \mathbb{E}_{i}[D]-A_{i} \operatorname{Cov}\left[E_{2 i}, D\right]\left(1-\frac{\beta_{i i}}{\beta_{i}}\right)+P_{1} r_{i}\left(1+\operatorname{sgn}\left(\Delta X_{1 i}\right) \tau\right)\right) \frac{d X_{1 i}}{d \tau}\right] d F(i),
$$

where $r_{i} \equiv \frac{\operatorname{Var}[D]}{\operatorname{Var}_{i}[D]}, \beta_{i i} \equiv \frac{\operatorname{Cov}_{i}\left[E_{2 i}, D\right]}{\mathbb{V} a r_{i}[D]}$ and $\beta_{i} \equiv \frac{\operatorname{Cov}\left[E_{2 i}, D\right]}{\mathbb{V} a r[D]}$. Note that $r_{i} \in(0, \infty)$ and $\beta_{i}, \beta_{i i} \in(-\infty, \infty)$.

b) Under assumption [NR], the optimal tax when investors disagree about second moments is given by:

$$
\tau^{*}=\frac{\int\left(r_{i} \mathbb{E}_{i}[D]+A_{i} \operatorname{Cov}\left[E_{2 i}, D\right]\left(1-\frac{\beta_{i i}}{\beta_{i}}\right)\right) \frac{d X_{1 i}}{d \tau} d F(i)}{P_{1} \int\left(r_{i}\left(1+\operatorname{sgn}\left(\Delta X_{1 i}\right)\right)\right) \frac{d X_{1 i}}{d \tau} d F(i)}
$$

Proof. See appendix A.

The formula for the optimal tax now incorporates hedging needs and modifies the weights given to investors' beliefs. An investor with correct beliefs about second moments has $r_{i}=1$ and $\beta_{i i}=\beta_{i}$; in that case, we recover (13). When investors perceive a high variance, that is, $r_{i}$ is close to 0 , they receive less weight in the optimal tax formula. The opposite occurs when they perceive a low variance. Intuitively, lower perceived variances amplify distortions in expected payoffs, and viceversa.

As in the baseline model, the planner does not need to know the value of $\mathbb{E}[D]$ to implement the optimal tax. However, if investors hold wrong beliefs about their hedging needs, the planner needs to know explicitly the magnitude of the mistake. Intuitively, there is no mechanism in the model which cancels out the mistakes in hedging made by investors. The sign of the optimal tax depends directly on the errors made by investors when hedging.

There are two interesting parameters restrictions. First, when investors with correct expected payoffs and hedging betas, that is $\frac{\beta_{i i}}{\beta_{i}}=1$, disagree about variances, the optimal tax $\tau^{*}$ turns out to be:

$$
\tau^{*}=\frac{\mathbb{E}[D] \int r_{i} \frac{d X_{1 i}}{d \tau} d F(i)}{P_{1} \int r_{i}\left(1+\operatorname{sgn}\left(\Delta X_{1 i}\right)\right) \frac{d X_{1 i}}{d \tau} d F(i)}
$$

The dispersion of variances, given by $\operatorname{Cov}_{F, \mathcal{T}}\left[r_{i}, \frac{d X_{1 i}}{d \tau}\right]$, determines now the sign of the optimal tax. When $r_{i}$ is constant (although not necessarily equal to one), the optimal tax becomes zero. This reinforces 
the intuition that belief dispersion is what matters for optimal taxes in an exchange economy. Intuitively, when buyers, with $\frac{d X_{1 i}}{d \tau}<0$, are relatively aggressive, that is, $r_{i}$ is large, they are buying too much of the risky asset, so $\operatorname{Cov}_{F, \mathcal{T}}\left[r_{i}, \frac{d X_{1 i}}{d \tau}\right]$ is negative and the optimal tax is positive, and viceversa.

Second, when agents have correct beliefs about the mean and the variance of expected returns, but hedge incorrectly, the optimal tax becomes:

$$
\tau^{*}=\frac{\operatorname{Var}[D] \int A_{i}\left(\beta_{i}-\beta_{i i}\right) \frac{d X_{1 i}}{d \tau} d F(i)}{P_{1} \int \operatorname{sgn}\left(\Delta X_{1 i}\right) \frac{d X_{1 i}}{d \tau} d F(i)}
$$

The optimal tax now has the opposite sign of $\operatorname{Cov}_{F, \mathcal{T}}\left[A_{i}\left(\beta_{i}-\beta_{i i}\right), \frac{d X_{1 i}}{d \tau}\right]$. Intuitively, when buyers, with $\frac{d X_{1 i}}{d \tau}<0$, overestimate their need for hedging and end up buying too much of the risky asset this occurs when $\beta_{i}-\beta_{i i}<0-$ the optimal tax is positive, and viceversa.

\subsection{Asymmetric taxes/Multiple tax instruments}

In the baseline model, the only instrument available to the planner is a single linear financial transaction tax which applies symmetrically to all investors. However, the planner could set different (linear) taxes for buyers and sellers. Or, at least theoretically, even investor-specific taxes. In general, more sophisticated policy instruments bring the outcome of the planner's problem closer to the first-best, at the cost of increasing informational requirements.

\section{Asymmetric taxes on buyers versus sellers}

Assume now that buyers pay a linear tax $\tau_{B}$ in the dollar volume of the transaction while sellers pay $\tau_{S}$. Hence, total tax revenue is given by $\left(\tau_{B}+\tau_{S}\right) P_{1}\left|\Delta X_{1 i}\right|$. Outside of the inaction region, the optimal portfolio demand is given by:

$$
X_{1 i}=\frac{\mathbb{E}_{i}[D]-A_{i} \operatorname{Cov}\left[E_{2 i}, D\right]-P_{1}\left(1+\mathbb{I}\left[\Delta X_{1 i}>0\right] \tau_{B}+\mathbb{I}\left[\Delta X_{1 i}<0\right] \tau_{S}\right)}{A_{i} \operatorname{Var}[D]},
$$

where $\mathbb{I}[\cdot]$ denotes the indicator function. This expression differs from (5) in that buyers now face a different tax than sellers. The equilibrium price is a natural extension of the baseline model - see appendix A.

\section{Proposition 8. (Asymmetric taxes on buyers versus sellers)}

a) Under assumption [NR], the pair of optimal taxes $\left\{\tau_{B}^{*}, \tau_{S}^{*}\right\}$ is characterized by the solution of the following system of non-linear equations:

$$
\tau_{B}^{*}+\tau_{S}^{*}=\frac{\int \frac{\mathbb{E}_{i}[D]}{P_{1}} \frac{d X_{1 i}}{d \tau_{B}} d F(i)}{\int_{\mathcal{B}} \frac{d X_{1 i}}{d \tau_{B}} d F(i)}, \quad \tau_{B}^{*}+\tau_{S}^{*}=\frac{\int \frac{\mathbb{E}_{i}[D]}{P_{1}} \frac{d X_{1 i}}{d \tau_{S}} d F(i)}{\int_{\mathcal{B}} \frac{d X_{1 i}}{d \tau_{S}} d F(i)}
$$

Proof. See appendix A.

The economic forces that shape the optimal values for $\tau_{B}^{*}$ and $\tau_{S}^{*}$ are the same as in the baseline model. Once again, the truth is irrelevant for the optimal policy. This shows that, as long as [NR] holds, 
the use of more sophisticated policy instruments does not require knowledge of the actual distribution of payoffs. Intuitively, the change in portfolio allocation induced by a marginal change in any instrument must cancel out in the aggregate. Equation (22) provides intuition for why all taxes in the baseline model are divided by 2 ; in that case, there exists a single optimality condition and $2 \tau^{*}=\tau_{B}^{*}+\tau_{S}^{*}$.

As long as there are more than two investors, this system has at least a solution. When there are two investors, the system is indeterminate and only the sum $\tau_{B}^{*}+\tau_{S}^{*}$ is pinned down. In that case, $\tau_{B}^{*}+\tau_{S}^{*}=\frac{\mathbb{E}_{\mathcal{B}}[D]-\mathbb{E}_{\mathcal{S}}[D]}{P_{1}}$.

\section{Individual taxes/First-best}

Assume now that the planner can set investor specific taxes. This is an interesting theoretical benchmark, despite being completely unrealistic. For simplicity, I now assume that there is a finite number $N$ of (types of) investors in the economy.

\section{Proposition 9. (Individual taxes/First-best)}

a) The first-best can be implemented with a set of investor specific taxes given by:

$$
\tau_{i}^{*}=\operatorname{sgn}\left(\Delta X_{1 i}\right) \frac{\mathbb{E}_{i}[D]-F}{P_{1}}, \forall i=1, \ldots, N,
$$

where $F$ is any real number; a natural choice for $F$ is $\mathbb{E}[D]$.

b) Under assumption [NR], the planner only needs $N-1$ taxes to implement the first-best in an economy with $N$ investors. If $[N R]$ does not hold, $N$ taxes are needed.

Proof. See appendix A.

Proposition 9a follows standard Pigovian logic. The planner sets optimal individual taxes so that investors portfolio choices are set as if there were a single return distribution. Note that the planner can use any belief $F$ to implement the first-best allocation, as long as it is the same for all agents. In a production economy, the natural choice would be $F=\mathbb{E}[D]$. Finally, because $P_{1}$ is a function of all taxes, equation (23) also defines a system of non-linear equations.

Proposition $9 \mathrm{~b}$ shows that the first-best could be implemented with $N-1$ taxes when [NR] holds. This occurs because the risky asset is in fixed supply. The logic behind this result is similar to Walras' law. For instance, when $N=2$, a single tax which modifies directly the allocation of one of the investors necessarily changes the allocation of the other one through market clearing.

\section{Dynamic Model with General Utility and Arbitrary Disagreement}

This section extends the main results to a dynamic environment with investors who face a non-trivial consumption choice between periods, have general utility specifications, and disagree about probability distributions in an arbitrary way. These results contrast with the baseline model, which only features a portfolio allocation problem, CARA utility and disagreement about finite dimensional moments of the distribution of dividends. 
Three insights emerge from the more general model. First, the expression for the optimal tax derived under CARA utility and normal returns is recovered after approximating the optimal tax formula around the case in which investors' marginal utilities are approximately constant. Hence, the tractable CARA-Normal results can be seen as a local approximation to more general models in which income effects are not particularly strong. ${ }^{31}$

Second, controlling for the level of static disagreement, a lower optimal tax is needed when forwardlooking investors buy and sell frequently. However, a higher optimal tax is needed when forwardlooking investors trade persistently in the same direction. ${ }^{32}$

Third, even for a planner who respects investors' beliefs, there may exist a rationale for taxation when markets are incomplete.

The time-series covariance between price sensitivities with respect to a tax change and marginal utility weighted changes in net trading positions becomes the welfare relevant sufficient statistic price volatility (an often used measure in policy discussions) has no correspondence with welfare.

\subsection{Equilibrium given taxes}

Environment Time is discrete, the economy lasts $T$ periods, with $t=\{1, \ldots, T\}$ and there is a unit measure of investors. Investors are indexed by $i$, and they are distributed according to a probability distribution $F$. They maximize time-separable expected utility of consumption with an arbitrary (well behaved) flow utility function $U_{i}\left(C_{t i}\right)$ and a discount factor $\beta$, identical for simplicity to all investors. Unlike in the baseline model, investors now decide how much to consume in every period.

Markets are incomplete. There exists a riskless asset in elastic supply with a (constant) gross interest rate of $R$. There is a single risky asset with an exogenous fixed supply $Q$. Every investor holds initially $X_{0 i}$ shares of the risky asset, such that $\int X_{0 i} d F(i)=Q$. The risky asset yields a stochastic dividend $D_{t}$ per period. Every investor receives a stochastic endowment $E_{t i}$ every period. Dividends and endowments follow arbitrary probability distributions. In appendix B, I show how the results extend to the case of endogenous labor supply/static choice variable.

I model investors' beliefs as a change of measure with respect to the actual probability measure, which (jointly) determines the realization of all random variables — dividends and endowments - in the model. For an investor $i$, his conditional beliefs at a period $t$ about $t+1$ uncertainty are determined by a Radon-Nikodym derivative $Z_{t i}$, which is absolutely continuous with respect to the true probability distribution. The random variables $\left\{Z_{t i}\right\}$ capture in a general form any type of discrepancy between the probability assessments used by the planner and the ones used by the investors. Investors' beliefs about future events are predetermined and dogmatic, and do not change when time and uncertainty unfold.

\footnotetext{
${ }^{31}$ This result is related to the classic Arrow-Pratt approximation (Arrow (1971), Pratt (1964)), which shows that the solution to the portfolio problem with CARA utility and normal distribution of returns is the correct approximation for small gambles. This is also an approximation to continuous time diffusion models. See Ingersoll (1987), Huang and Litzenberger (1988) or Gollier (2001) for textbook treatments of these results.

${ }^{32}$ Tobin (1978) already acknowledges that the kind of transaction tax analyzed in this paper would have disproportionate effects over short horizon investments. The planner in this paper endogenously exploits this property of the tax when setting the optimal policy.
} 
It is straightforward to allow for beliefs that evolve stochastically, as long as their evolution does not depend on endogenous variables.

I continue to restrict the set of policy measures to a constant linear transaction tax under commitment. The use of time varying taxes raises a different set of concerns - related to credibility and commitment - which I do not address in this paper. ${ }^{33}$

An investor $i$ solves the problem:

$$
\max _{C_{t i}, X_{t i}, Y_{t i}} \mathbb{E}_{i}\left[\sum_{t=1}^{T} \beta^{t-1} U_{i}\left(C_{t i}\right)\right]
$$

Subject to a period by period budget constraint, given by:

$$
C_{t i}=E_{t i}+X_{t-1 i}\left(P_{t}+D_{t}\right)-\tau P_{t}\left|\Delta X_{t i}\right|+T_{t i}-X_{t i} P_{t}+R Y_{t-1 i}-Y_{t i} \quad \text { for periods } t=1, \ldots, T-1 \text {, }
$$

where $X_{t i}$ denotes the number of shares of the risky asset held and $Y_{t i}$ denotes the dollar amount invested in the riskless asset. In the last period $T$, investors consume $C_{T i}=E_{T i}+X_{T-1 i} D_{T}+R Y_{T i}$. Note that every investor uses his individual expectation $\mathbb{E}_{i}$ to solve his optimization problem.

For simplicity, I assume that tax revenue is returned exactly to those investors who bore them, that is $T_{t i}=\tau P_{t}\left|\Delta X_{t i}\right|$. Because income effects now matter to determine portfolio allocations, this assumption is now restrictive, unlike in the baseline model. However, as long as income effects do not play a major role in determining portfolio decisions - which should be the case if the size of the tax is not too large as a proportion of each investor's wealth - the results should not vary with respect to the case with arbitrary rebates.

The equilibrium definition is standard - see appendix A. I assume that the problem is sufficiently well behaved and that there exists a competitive equilibrium. ${ }^{34}$ I also assume that asset prices are strictly positive at all periods and that the planner's problem is differentiable. Lastly, for the interpretation of the normative results, I assume that the counterparts of lemmas 1 and 2 hold.

Although it is impossible to provide analytical characterizations for equilibrium portfolio demands and prices, the optimal tax formula can still be expressed as a function of a small number of sufficient statistics. 35

Equilibrium characterization The Euler equations for the riskless and the risky asset, given by (26) and (27) characterize investors' optimal portfolio allocation. The Euler equation for the risky asset only

\footnotetext{
${ }^{33}$ There is scope to extend the results of this paper to a dynamic Ramsey environment with time varying policies, along the lines of Lucas and Stokey (1983), Aiyagari et al. (2002) or Chari and Kehoe (1999). However, the practical feasibility of time-varying taxation in this environment is questionable. In any case, even the optimal constant linear tax characterized here suffers from time inconsistency. There is also scope to explore within this framework transaction taxes that depend on the holding period.

${ }^{34}$ Because markets are incomplete, only generic existence is guaranteed. See for instance, Hart (1974, 1975), Geanakoplos and Polemarchakis (1986) or Magill and Quinzii (2002).

${ }^{35}$ Solving this model numerically is a complex task. The existence of inaction regions prevents the use of local solution methods. Moreover, investors must keep track of their two individual state variables (wealth and holdings of the risky asset), in addition to the distributions of these variables in the population of investors. The simplest model which captures different frequencies on trading would require 3 investors and at least five endogenous state variables (six if production is allowed).
} 
holds when investors decide to buy or sell a non-zero amount; if an investor decides not to trade the risky asset, (27) is replaced by $X_{t i}=X_{t-1 i}$.

$$
\begin{gathered}
U_{i}^{\prime}\left(C_{t i}\right)=\beta R \mathbb{E}_{t i}\left[U_{i}^{\prime}\left(C_{t+1 i}\right)\right] \\
U_{i}^{\prime}\left(C_{t i}\right) P_{t}\left(1+\operatorname{sgn}\left(\Delta X_{t i}\right) \tau\right)=\beta \mathbb{E}_{t i}\left[U_{i}^{\prime}\left(C_{t+1 i}\right)\left(D_{t+1}+P_{t+1}\left(1+\operatorname{sgn}\left(\Delta X_{t+1 i}\right) \tau\right)\right)\right]
\end{gathered}
$$

I use the notation $\mathbb{E}_{t i}$ to denote the period $t$ expectation by an investor $i$. The intuition behind the optimality conditions is standard. Shifting consumption between periods with either the riskless or the risky asset must leave each investor indifferent at the margin. In the case of the risky asset, the cost of the transaction tax must be taken into consideration. It is useful to rewrite (26) and (27) as:

$$
\begin{gathered}
U_{i}^{\prime}\left(C_{t i}\right)-\beta R \mathbb{E}_{t}\left[U_{i}^{\prime}\left(C_{t+1 i}\right)\right]=\Lambda_{t, \text { safe }} \\
\text { where } \Lambda_{t, \text { safe }}=\beta R \operatorname{Cov}_{t}\left[Z_{t+1 i}, U_{i}^{\prime}\left(C_{t+1 i}\right)\right] \\
U_{i}^{\prime}\left(C_{t i}\right) P_{t}-\beta \mathbb{E}_{t}\left[U_{i}^{\prime}\left(C_{t+1 i}\right)\left(D_{t+1}+P_{t+1}\right)\right]=\Lambda_{t, \text { risky, }} \\
\text { where } \Lambda_{t, \text { risky }}=-U_{i}^{\prime}\left(C_{t i}\right) P_{t} \operatorname{sgn}\left(\Delta X_{t i}\right) \tau+\beta \mathbb{E}_{t i}\left[U_{i}^{\prime}\left(C_{t+1 i}\right) P_{t+1} \operatorname{sgn}\left(\Delta X_{t+1 i}\right)\right] \tau \\
+\beta \operatorname{Cov}_{t}\left[Z_{t+1 i}, U_{i}^{\prime}\left(C_{t+1 i}\right)\left(D_{t+1}+P_{t+1}\right)\right]
\end{gathered}
$$

Both terms on the right hand side of equations (28) and (29) represent the wedges on the optimal portfolio choice condition from a planner's perspective. The wedge in (28) captures the distortion in precautionary savings induced by having incorrect beliefs. Intuitively, if an investor weighs more those states in which he has higher marginal utility $\operatorname{Cov}_{t}\left[Z_{t+1 i}, U_{i}^{\prime}\left(C_{t+1 i}\right)\right]>0$, he perceives as optimal to save more, and viceversa.

The wedge in (29) has three terms. The first term is the distortion caused by imposing a transaction tax in period $t$. I refer to this term as the static effect of the tax. The second term is the distortion caused by imposing a transaction in period $t+1$. I refer to this term as the dynamic or forward-looking effect of the tax. The third term captures the distortion generated by holding incorrect beliefs. Intuitively, if an investor weighs more those states in which the payoff of the risky asset is higher - especially if those are high marginal utility states - , that is, $\operatorname{Cov}_{t}\left[Z_{t+1 i}, U_{i}^{\prime}\left(C_{t+1 i}\right)\left(D_{t+1}+P_{t+1}\right)\right]>0$, he finds more attractive to buy more of it, and viceversa. The planner makes use of both the static and the dynamic effect of the tax to close the wedges generated by holding incorrect beliefs.

The combination of (26) and (27) with the market clearing conditions fully characterize the equilibrium. Unlike in the CARA-Normal model, now it is impossible to characterize equilibrium prices and portfolio allocations explicitly.

\subsection{Optimal tax policy}

As in the baseline model, social welfare - denoted again by $V$ - can be written as the sum of the indirect utility for each investor $i$, under the planner's measure, that is:

$$
V(\tau)=\int \lambda_{i} V_{i} d F(i)
$$


where $V_{i}$ denotes indirect utility from the planner's perspective, that is:

$$
V_{i}=\mathbb{E}\left[\sum_{t=1}^{T} \beta^{t-1} U_{i}\left(C_{t i}\right)\right]
$$

With a per-period budget constraint $C_{t i}=E_{t i}+X_{t-1 i}\left(P_{t}+D_{t}\right)-X_{t i} P_{t}+R Y_{t-1 i}-Y_{t i}$ and where $X_{t i}$ and $Y_{t i}$ are determined by (26) and (27). Note that the planner internalizes the transfer rebated to each investor and uses his own probability distribution to assess probabilities. I use the notation $\mathbb{E}[\cdot]$ to denote the unconditional expectation held by the planner.

The marginal change in welfare induced by a marginal change on the tax $\tau$ for a given investor $i$ can be written as:

$$
\frac{d V_{i}}{d \tau}=\mathbb{E}\left[\sum_{t=1}^{T} \beta^{t-1}\left(-\Lambda_{t, \text { risky }} \frac{d X_{t i}}{d \tau}-\Lambda_{t, \text { safe }} \frac{d Y_{t i}}{d \tau}-U_{i}^{\prime}\left(C_{t i}\right) \Delta X_{t i} \frac{d P_{t}}{d \tau}\right)\right]
$$

where $\Lambda_{t, \text { risky }}$ and $\Lambda_{t, \text { safe }}$ are defined in (29) and (28). A marginal tax change modifies the portfolio allocation of both the riskless and the risky asset. The change in welfare induced by these movements is determined by the pre-existing wedges in the respective Euler equations. For instance, if the unconstrained Euler equation from the planner's perspective holds for the risky asset, that is $U_{i}^{\prime}\left(C_{t i}\right) P_{t}=\beta \mathbb{E}_{t}\left[U_{i}^{\prime}\left(C_{t+1 i}\right)\left[P_{t+1}+D_{t+1}\right]\right]$, the marginal change in welfare induced by varying the tax rate through a change in holdings of the risky asset is zero. In addition to changes in portfolio allocations, a tax change modifies equilibrium prices, generating terms-of-trade effects, which would benefit buyers (sellers) when prices go down (up).

To characterize sharper results, I introduce the following assumption.

\section{Assumption 4. [CMU/NR] (Constant Marginal Utility/No Redistributional motive)}

a) Marginal utility is (approximately) constant across all periods and states, that is $U_{i}^{\prime}\left(C_{t i}\right) \approx 1$.

b) The planner is a strict utilitarian, that is $\lambda_{i}=1, \forall i$.

In part a), assumption [CMU/NR] constrains investors marginal utility of consumption to be roughly constant. In part b), assumption [CMU/NR] further imposes that the planner does not wish to redistribute resources from one group of investors to another one. In the dynamic context, assumption [CMU/NR] is more restrictive than assumption [NR] in the baseline model, because it removes insurance considerations in addition to redistributional considerations. More generally, we can think that assumption [CMU/NR] is valid when the risks borne by investors are not too large in comparison to their willingness to assume risk. This is always the case when they are approximately risk neutral. I separately analyze the effects of taxes on insurance in the next subsection 6.3.

To ease the exposition, I normalize $R=1$ and $\beta=1$ for the rest of this section.

Main results Proposition 10 contains the main results of this section. I apply directly assumption $[\mathrm{CMU} / \mathrm{NR}]$ but derive the results for the general case in appendix A.

\section{Proposition 10. (Marginal effect on welfare/Optimal tax)}


a) Under assumption [CMU/NR], the marginal change in social welfare from varying the financial transaction tax is given by:

$$
\frac{d V}{d \tau}=\mathbb{E}\left[\sum_{t=1}^{T} \int\left(\begin{array}{c}
P_{t} \operatorname{sgn}\left(\Delta X_{t i}\right) \tau-\mathbb{E}_{t i}\left[P_{t+1} \operatorname{sgn}\left(\Delta X_{t+1 i}\right)\right] \tau \\
-\operatorname{Cov}_{t}\left[Z_{t+1 i},\left(D_{t+1}+P_{t+1}\right)\right]
\end{array}\right) \frac{d X_{t i}}{d \tau} d F(i)\right]
$$

b) Under assumption [CMU/NR], the optimal financial transaction tax is given by:

$$
\tau^{*}=\frac{\mathbb{E}\left[\sum_{t=1}^{T} \int \mathbb{E}_{t i}\left[D_{t+1}+P_{t+1}\right] \frac{d X_{t i}}{d \tau} d F(i)\right]}{\mathbb{E}[\sum_{t=1}^{T} P_{t} \int \operatorname{sgn}\left(\Delta X_{t i}\right) \underbrace{\left(1-\kappa_{t i}\right)}_{\begin{array}{c}
\text { forward -looking } \\
\text { correction }
\end{array}} \frac{d X_{t i}}{d \tau} d F(i)]}
$$

where $\kappa_{t i} \equiv \mathbb{E}_{t i}\left[\frac{P_{t+1}}{P_{t}} \operatorname{sgn}\left(\Delta X_{t i}\right) \operatorname{sgn}\left(\Delta X_{t+1 i}\right)\right]$.

Proof. See appendix A.

The optimal tax only depends on expected returns and portfolio tax sensitivities when agents are approximately risk neutral, independently of the shape of belief heterogeneity, determined by $Z_{t i}$. This approximation shows that the optimal tax in the CARA-Normal model captures effects of first-order importance.

There are two main differences between equations (31) and (32) and their counterparts in the baseline model. First, the probability distribution used by the planner does not drop out of the optimal formula even under assumption [CMU/NR]. In a period-by-period/state-by-state basis, the relative belief distortion is still the key sufficient statistic for welfare, since it determines investors' risk exposures. However, the planner must use a probability distribution to weigh the likelihood of reaching different states. The belief of the planner only matters to determine these weights, but it does not determine directly the sign of the tax. For instance, if static taxes are positive at every state, independently of the beliefs chosen by the planner, $\tau^{*}$ must be positive too. If optimal static tax is constant in every period, the belief used by the planner does not matter.

Second, a marginal change in the tax rate now has an additional forward looking effect, captured by the term $\left(1-\kappa_{t i}\right)$ in the denominator. ${ }^{36}$ Intuitively, the planner takes into account that a marginal tax change further modifies period $t$ portfolio allocations because investors anticipate paying the tax in future. To provide intuition for this term, assume a) that $\mathbb{E}_{t i}\left[P_{t+1} / P_{t}\right]=1, \forall i$ and b) that $\operatorname{sgn}\left(\Delta X_{t+1 i}\right)$ is the same in all future $t+1$ states. In that case, $\left(1-\kappa_{t i}\right)$ can take three values:

$$
1-\kappa_{t i}=\left\{\begin{array}{lll}
2 & \text { if } \operatorname{sgn}\left(\Delta X_{t i}\right) \operatorname{sgn}\left(\Delta X_{t+1 i}\right)<0 & \left(\kappa_{t i}=-1\right) \\
1 & \text { if } \operatorname{sgn}\left(\Delta X_{t+1 i}\right)=0 & \left(\kappa_{t i}=0\right) \\
0 & \text { if } \operatorname{sgn}\left(\Delta X_{t i}\right) \operatorname{sgn}\left(\Delta X_{t+1 i}\right)>0 & \left(\kappa_{t i}=1\right)
\end{array}\right.
$$

Intuitively, when investors trade at period $t$ and in the opposite direction in all possible states at $t+1$, the same tax distorts twice the time $t$ portfolio decision. When investors buy-and-hold, only the time

\footnotetext{
${ }^{36}$ Although the tax formula is written as if only one period ahead trading matters, by combining Euler equations, all forward looking effects are actually considered. This characterization relies on the fact that investors have a consumption choice.
} 
$t$ distortion modifies the portfolio decision - this is exactly what happens in the static model. Finally, when investors trade in the same direction at periods $t$ and $t+1$, a transaction tax does not affect at all period $t$ portfolio decision. A persistent buyer (seller) is aware that every (marginal) share purchased at $t$ has the extra benefit of saving the $t+1$ tax, rendering the tax irrelevant.

In general, $\kappa_{t i}$ is given by the expected value of these effects over the possible realizations of $\operatorname{sgn}\left(\Delta X_{t+1 i}\right)$. The logic described here extends to the case with $\mathbb{E}_{t i}\left[P_{t+1} / P_{t}\right] \neq 1$. Because the tax is paid on the dollar value of the transaction, investors tilt their purchasing decision towards periods with lower prices. For instance, when prices are steeply rising, a persistent buyer has a further incentive to buy early. Therefore, expected price growth can amplify or dampen the forward-looking effect. ${ }^{37}$

The optimal tax formula can be rewritten in a more intuitive way. See the following corollary to proposition 10 .

\section{Corollary. (Optimal dynamic tax formula)}

a) The optimal tax can be written as a corrected weighted average of static taxes, that is:

$$
\tau^{*}=\sum_{t=1}^{T} \mathbb{E}\left[\omega_{t} f_{t} \tau_{t}^{*}\right],
$$

where the random variables weights $\omega_{t}$ and optimal static taxes $\tau_{t}^{*}$ are defined in appendix $A . f_{t}$ is defined as $f_{t} \equiv \frac{1}{\int l_{t i}\left(1-\kappa_{t i}\right) d F(i)}$, where $l_{t i}$ are a set of weights - defined in appendix $A$ - which add up to one, that is $\int l_{t i} d F(i)=1$. The sum of weights $\omega_{t}$ also adds up to one, that is, $\sum_{t=1}^{T} \mathbb{E}\left[\omega_{t}\right]=1$. Dynamic corrections $f_{t}$ and static taxes $\tau_{t}^{*}$ can in principle take any value.

b) When most investors who buy in a given period/state become on average sellers in the next one (and viceversa), $f_{t}<1$. In that case, controlling for the level of static disagreement, the optimal tax in a dynamic model is lower than in a static model. When most investors are persistent buyers (or sellers), $f_{t}>1$ and the optimal tax is higher than in a static model.

Proof. See appendix A.

If $f_{t}=1$ at all periods and states, the optimal tax becomes a weighted average of static taxes, with weights determined by relative portfolio responses. This intuitive result is similar to the multiple risky asset case. However, the interesting cases occur when $f_{t} \neq 1$. Less weight is given to states in which $f_{t}<1$, because forward-looking investors who trade on opposite directions at high frequencies are more responsive to the tax. The opposite occurs when $f_{t}>1$, in which most of the investors are persistent buyers/sellers. Hence, for a given amount of disagreement induced trading - measured in the form of static taxes - , a lower optimal tax is required when forward-looking investors alternate between being buyers and sellers. On the contrary, a higher tax is optimal if they are persistent buyers. Intuitively, very large taxes are needed to dissuade investors who are planing to build long-term positions over time.

Therefore, the planner exploits the fact that trades at high frequencies are particularly discouraged when solving for the optimal tax. In other words, the planner endogenously uses the well known fact

\footnotetext{
${ }^{37}$ For the interpretation of the results, I implicitly assume that this effect is never powerful enough to turn $\left(1-\kappa_{t i}\right)$ negative. See appendix A for a more detailed discussion.
} 
that small trading costs can have large effects in dynamic models to design optimal policies. Trading dynamics directly influences the magnitude of the tax - through the set of weights $\omega_{t}$ - but not necessarily its sign (i.e., if all $\tau_{t}^{*}$ are positive, $\tau^{*}$ must be positive too). Only the existence of belief distortions provides a rationale for corrective policies.

Assuming that $\tau_{t}^{*}=\tau_{\text {static }}^{*}$ is constant at all periods/states and that investors alternate between buying and selling at all periods, a simple rule-of-thumb suggests that the optimal tax in a dynamic environment is half of the optimal static tax (as described for instance in equation (13)), that is:

$$
\tau_{\text {dynamic }}^{*} \approx \frac{1}{2} \tau_{\text {static }}^{*}
$$

Intuitively, because investors pay the tax twice, both when buying and selling, only half of the static tax is required. This "one-half logic" is tied to the particular discrete time formulation and the fact that investors have a consumption decisions; alternative models - for example, a continuous time model without intermediate consumption - may deliver different quantitative (but not qualitative) answers.

Finally, despite capturing dynamics, this paper does not model explicitly the role of modern high frequency trading. In the context of this model, that profile of investors would become instantly inframarginal for any tax, even an arbitrarily small one. Hence, if they play an essential role in financial markets, that would provide an additional argument for the policymaker against any tax. If continuous trading were replaced by alternative trading arrangements - like frequent auctions, as proposed by Budish, Cramton and Shim (2013) — the logic of this paper would still apply.

\subsection{Price volatility arguments revisited}

To conclude this section, I refute a common verbal argument that suggests that transaction taxes are welfare improving because they reduce price volatility. Why should a variance - volatility - be the natural variable to target from a policy perspective? I show here that, for a planner which respects investors' beliefs, as long as markets are incomplete, there may be scope to have a corrective (positive or negative) tax. ${ }^{38}$ The goal of this tax/subsidy is to provide insurance through the appropriate movements of asset prices in different states/periods.

The relevant question is not whether transaction taxes make asset prices more or less volatile; the correct question is whether transaction taxes modify asset prices at the right times. Price volatility may be good, if it provides insurance. Any debate on the subject of transaction taxes should move away from the question of whether prices are too volatile, to ask: a) whether investors are holding the right amount of risk or $b$ ) whether asset prices provide the correct amount of insurance.

From now on, I assume either that a) all investors hold fundamental beliefs or b) the planner respects individual beliefs when calculating social welfare. Conceptually, this analysis is the dynamic counterpart to the results relating to Harberger (1964) in subsection 3.4. In the static case, any tax policy is welfare reducing, since there is no scope for improving insurance. That is not the case here.

\footnotetext{
${ }^{38}$ For most of the paper, I focus on the effects of the tax in discouraging non-fundamental trading. In this subsection, I only focus on the role of the tax in providing insurance. For clarity, I study both mechanisms in isolation, although they naturally arise together and could potentially interact in interesting ways.
} 
A marginal tax change has first-order effects from a dynamic perspective as long as agents marginal utilities are not proportional.

Proposition 11. (Price volatility arguments revisited) a) The change in social welfare induced by a marginal change in the financial transaction tax is given by:

$$
\frac{d V}{d \tau}=\int \lambda_{i} \sum_{t=1}^{T} \mathbb{E}\left[Z_{i}\left(\xi_{t i} \frac{d X_{t i}}{d \tau} \tau-U_{i}^{\prime}\left(C_{t i}\right) \Delta X_{t i} \frac{d P_{t}}{d \tau}\right)\right] d F(i),
$$

where I denote by $Z_{i}$ the Radon-Nikodym derivative representing individual investors' beliefs from a $t=1$ perspective and define $\xi_{t i} \equiv U_{i}^{\prime}\left(C_{t i}\right) P_{t} \operatorname{sgn}\left(\Delta X_{t i}\right)\left(1-\mathbb{E}_{t i}\left[\frac{U_{i}^{\prime}\left(C_{t+1 i}\right)}{U_{i}^{\prime}\left(C_{t i}\right)} \frac{P_{t+1}}{P_{t}} \operatorname{sgn}\left(\Delta X_{t i}\right) \operatorname{sgn}\left(\Delta X_{t+1 i}\right)\right]\right)$.

b) The optimal tax is given by:

$$
\tau^{*}=\frac{\sum_{t=1}^{T} \mathbb{E}\left[\int \lambda_{i} Z_{i} U_{i}^{\prime}\left(C_{t i}\right) \Delta X_{t i} d F(i) \frac{d P_{t}}{d \tau}\right]}{\sum_{t=1}^{T} \mathbb{E}\left[\int \lambda_{i} Z_{i} \xi_{t i} \frac{d X_{t i}}{d \tau} d F(i)\right]}
$$

Proof. See appendix A.

A marginal tax change affects welfare once again through portfolio allocations and price changes. To focus on insurance, I do not impose assumption [CMU/NR]. ${ }^{39}$ Hence, the terms-of-trade effects, which cancel out in a static setup, are relevant in a dynamic model as long as $U_{i}^{\prime}\left(C_{t i}\right)$ are not perfectly proportional across investors - this is always the case, unless markets are complete. When $\tau=0$, portfolio effects become second-order - due to the envelope theorem-, but price changes remain firstorder, since they affect all traded shares.

The numerator of the optimal tax determines the marginal change in welfare when $\tau=0$, as in proposition 1c, at the same time that it pins down the sign of the optimal tax. It is a weighted average across periods and states of the product of price derivatives $\frac{d P_{t}}{d \tau}$ with the following cross sectional expectation:

$$
\zeta(\tau) \mathbb{E}_{F, \mathcal{T}}\left[\lambda_{i} Z_{i} U_{i}^{\prime}\left(C_{t i}\right) \Delta X_{t i}\right]=-\zeta(\tau) \operatorname{Cov}_{F, \mathcal{T}}\left[\lambda_{i} Z_{t i} U_{i}^{\prime}\left(C_{t i}\right), \Delta X_{t i}\right]
$$

where $\zeta(\tau)$ denotes the fraction of active investors in a given state. To ease the interpretation, assume a) that $\lambda_{i}=1$ and $\mathrm{b}$ ) that the denominator of $\tau^{*}$ is negative - the natural case. Under those assumptions, the optimal tax is positive when:

$$
\mathbb{E}\left[\zeta(\tau) \operatorname{Cov}_{F, \mathcal{T}}\left[Z_{t i} U_{i}^{\prime}\left(C_{t i}\right), \Delta X_{t i}\right] \frac{d P_{t}}{d \tau}\right]<0,
$$

The cross sectional covariance term is positive when buyers' marginal utilities are larger than sellers' marginal utilities at a given state. In that case, having $\frac{d P_{t}}{d \tau}<0$ calls for a positive tax and viceversa. Intuitively, the planner would like to favor the terms-of-trade of those agents with relatively higher marginal utilities. Assuming that the effect of the tax on the asset price is zero in expectation, that is

\footnotetext{
${ }^{39}$ However, imposing a) assumption [CMU/NR] and b) that investors trade in opposite directions at every period, that is, $f_{t}=\frac{1}{2}$, we can find the largest upper bound for the marginal welfare loss of a tax change, analogously to section 3.4. In that case, we can write $\left.\int \frac{d V_{i}}{d \tau}\right|_{\tau=\tilde{\tau}} d F(i)=4 \tilde{\tau} \sum_{t=1}^{T} \mathbb{E}\left[P_{t} \int_{i \in \mathcal{B}} \frac{d X_{1 i}}{d \tau}\right] d F(i)$. Intuitively, the welfare loss is now twice as large because it accounts for the forward-looking distortion.
} 
$\mathbb{E}\left[\frac{d P_{t}}{d \tau}\right]=0$ - this is a reasonable assumption, given lemma 1 in the baseline model - , the optimal tax is positive when the time-series covariance between the cross sectional covariance of marginal utility and net trade positions is negative, that is when:

$$
\operatorname{Cov}\left[\zeta(\tau) \operatorname{Cov}_{F, \mathcal{T}}\left[Z_{i} U_{i}^{\prime}\left(C_{t i}\right), \Delta X_{t i}\right], \frac{d P_{t}}{d \tau}\right]<0
$$

Observe that it is the covariance of price changes what matters for welfare, but not the variance. Price volatility induced by the tax, i.e. changes in $\frac{d P_{t}}{d \tau}$ with opposite sign in different states, could be welfare improving.

The results of this section show how to implement a linear tax with the goal of maximizing a given set of welfare weights. However, depending on the choice of $\lambda_{i}$, this may or may not generate a Pareto improvement. Although incomplete market economies are constrained inefficient, it is not obvious that a simple second best tool like a linear transaction tax is sufficient to create a Pareto improvement — more instruments may be needed. ${ }^{40}$ In appendix A, I provide sufficient conditions for a Pareto improvement for the case with two different (groups of) investors.

\section{Production}

The results derived so far rely on the assumption that assets are in fixed supply, which implies that aggregate consumption is exogenously determined. Starting from the baseline model, I now introduce two different forms of production to show how optimal policies change when production and investment are endogenous.

First, I analyze the Walrasian production case. In that situation, financial markets determine production by influencing the intertemporal decision between consumption and investment in a standard price-taking environment - this is the role explored in q-theory models, as Tobin (1969) and Hayashi (1982).

Second, I analyze the Hayekian production case. In that situation, financial markets determine production because they allow firm managers to learn useful information to guide production decisions from investors.

\subsection{Walrasian production}

Assumptions There is a new group of agents in the economy who were not present in the baseline model: identical competitive producers in unit measure. Producers are indexed by $k$ and maximize well-behaved time separable expected utility, with flow utility given by $U_{k}(\cdot)$. They have exclusive access to a technology $\Phi\left(S_{1 k}\right)$, which allows them to issue $S_{1 k}$ shares of the risky asset at $t=1 .{ }^{41}$ I refer

\footnotetext{
${ }^{40}$ See Geanakoplos and Polemarchakis (1986). The results of this section are related to the literature in pecuniary externalities due to incomplete markets. See, in different contexts, Gromb and Vayanos (2002), Lorenzoni (2008), Korinek (2009), He and Kondor (2012) and Davila (2011).

${ }^{41} \mathrm{~A}$ "tree" analogy can be helpful here. Assume that a share of the risky asset (i.e. a tree) entitles the owner to a dividend payment $D$ (fruit). Producers can plant new trees at a cost $\Phi\left(S_{1 k}\right)$, which they sell at a price $P_{1}$. They would be willing
} 
to $S_{1 k}$ as investment. The function $\Phi(\cdot)$ is increasing and strictly convex; that is, $\Phi^{\prime}(\cdot)>0, \Phi^{\prime \prime}(\cdot)>0$. To ease the exposition, I assume throughout that $\Phi\left(S_{1 k}\right)=\gamma_{1} S_{1 k}+\frac{\gamma_{2}}{2}\left(S_{1 k}\right)^{2}$, with $\gamma_{1}, \gamma_{2}>0$. By assuming that the price of the risky asset when $S_{1 k}=0$ is larger than $\gamma_{1}$, this technology guarantees that share issuance is strictly positive. Producers are initially endowed with $E_{1 k}$ units of consumption good (dollars) and can only borrow or save in the riskless asset at a (gross) rate $R=1$. Their endowment $E_{2 k}$ at $t=2$ is stochastic with an arbitrary distribution.

To avoid distortions in primary markets, the planner does not tax the issuance of new shares. Importantly, market clearing is now given by $\int X_{1 i} d F(i)=Q+S_{1 k}$. Total output at $t=2$ in this economy is endogenous and given by $D\left(Q+S_{1 k}\right)$.

Results: equilibrium characterization Producers thus maximize:

$$
\max _{C_{1 k}, C_{2 k}, S_{1 k}} U_{k}\left(C_{1 k}\right)+\mathbb{E}\left[U_{k}\left(C_{2 k}\right)\right]
$$

With budget constraints $Y_{k}+C_{1 k}=E_{1 k}+P_{1}^{s} S_{1 k}-\Phi\left(S_{1 k}\right)$ and $C_{2 k}=E_{2 k}+Y_{k}$, where $Y_{k}$ denotes the amount saved in the riskless asset and $P_{1}^{s}$ denotes the price faced by producers - the superscript $s$ stands for supply. The optimality conditions for producers are given by:

$$
\begin{gathered}
U_{k}^{\prime}\left(C_{1 k}\right)=\mathbb{E}\left[U_{k}^{\prime}\left(C_{2 k}\right)\right] \\
P_{1}^{s}=\Phi^{\prime}\left(S_{1 k}\right)
\end{gathered}
$$

The first condition is a standard Euler condition for the riskless asset. The second condition provides a supply curve for shares. Given the parametric assumption about $\Phi\left(S_{1 k}\right)$, it can be written as $P_{1}^{s}=\gamma_{1}+\gamma_{2} S_{1 k}$. Combining this upward sloping supply curve with the following downward sloping demand:

$$
P_{1}^{d}=\frac{\Gamma_{\text {num }}-A \mathbb{V a r}[D] S_{1 k}}{\Gamma_{\text {denom }}},
$$

where $\Gamma_{\text {num }}$ and $\Gamma_{\text {denom }}$ respectively denote the numerator and denominator in equation (7), allows us to pin down the equilibrium values of $P_{1}$ and $S_{1 k}$, given a tax $\tau$. Intuitively, because now the supply of shares is elastic, the equilibrium price per share of the risky asset has to be lower than in the $S_{1 k}=0$ case, to induce investors to hold a larger amount of aggregate risk.

Results: welfare analysis Accounting for producers, social welfare is now defined as:

$$
V(\tau)=\int \lambda_{i} V_{i} d F(i)+\lambda_{k} V_{k}
$$

where $V_{k}$ and $\lambda_{k}$ respectively denote the indirect utility and the welfare weight of producers. The change in producers' welfare induced by a marginal change in the tax is given by:

$$
\begin{aligned}
\frac{d V_{k}}{d \tau} & =U_{k}^{\prime}\left(C_{1 k}\right)\left[\frac{d P_{1}}{d \tau} S_{1 k}+\left[P_{1}-\Phi^{\prime}\left(S_{1 k}\right)\right] \frac{d S_{1 k}}{d \tau}-\frac{d Y_{k}}{d \tau}\right]+\mathbb{E}\left[U_{k}^{\prime}\left(C_{2 k}\right)\right] \frac{d Y_{k}}{d \tau} \\
& =\mathbb{E}\left[U_{k}^{\prime}\left(C_{2 k}\right)\right] \frac{d P_{1}}{d \tau} S_{1 k},
\end{aligned}
$$

to create trees until the marginal cost of producing a new tree $\Phi^{\prime}\left(S_{1 k}\right)$ equals the marginal benefit $P_{1}$. For consistency, any normalization concerning $Q$ must also normalize $\Phi(\cdot)$. The link between asset prices and real investment is less direct in a modern economy than in the economy modeled here. 
where the second line follows by substituting producers' optimality conditions. Intuitively, because producers do not pay taxes and invest optimally given prices, a marginal tax change only modifies their welfare through the terms-of trade on share issuance. When $P_{1}$ is high, producers enjoy a better deal selling shares than when $P_{1}$ is low. The envelope theorem eliminates from $\frac{d V_{k}}{d \tau}$ the direct effects caused by changes in producers portfolio or investment choices.

I now characterize marginal effects in welfare and optimal taxes. In proposition 12, I impose assumption [NR] directly, assuming that it also applies to producers' welfare.

\section{Proposition 12. (Marginal effect on welfare/Optimal tax)}

a) Under assumption [NR], the marginal change in social welfare from varying the financial transaction tax in an economy with production is given by:

$$
\frac{d V}{d \tau}=\int\left[\left(\mathbb{E}[D]-\mathbb{E}_{i}[D]\right)+\operatorname{sgn}\left(\Delta X_{1 i}\right) P_{1} \tau\right] \frac{d X_{1 i}}{d \tau} d F(i)
$$

b) Under assumption [NR], the optimal tax in an economy with production is given by:

$$
\tau^{*}=\frac{\int\left(\frac{\mathbb{E}[D]-\mathbb{E}_{i}[D]}{P_{1}}\right) \frac{d X_{1 i}}{d \tau} d F(i)}{-\int \operatorname{sgn}\left(\Delta X_{1 i}\right) \frac{d X_{1 i}}{d \tau} d F(i)}
$$

Proof. See appendix A.

Observe that, unlike proposition 1, the term corresponding to the planner's belief does not drop out of the problem. Market clearing now implies that $\int \frac{d X_{1 i}}{d \tau} d F(i)=\frac{d S_{1 k}}{d \tau}$, which can take any value, unlike in the baseline model, in which $\frac{d S_{1 k}}{d \tau}=0$ because of the fixed supply of assets. Given its conceptual importance, I state this result as a corollary of proposition 12.

Corollary. ( $\tau^{*}$ depends on planner's belief) As long as $\frac{d P_{1}^{d}}{d \tau} \neq 0$ (investors' total demand for shares varies with tax changes) and $\Phi^{\prime \prime}(\cdot) \neq 0$ (investment supply is responsive to price changes), the optimal financial transaction tax depends on the true payoff distribution. This occurs even when assumption [NR] holds.

In Walrasian production economies, the belief used by the planner to calculate welfare matters in general for the optimal policy. Intuitively, aggregate distortions in investors' beliefs now matter for welfare, since aggregate risk and output change through production.

The numerator of the optimal tax determines the marginal change in welfare when $\tau=0$, as in proposition 1c. It also pins down the sign of the optimal tax. It can be intuitively decomposed in two terms:

$$
\int\left(\mathbb{E}[D]-\mathbb{E}_{i}[D]\right) \frac{d X_{1 i}}{d \tau} d F(i)=\zeta(\tau)[\underbrace{\operatorname{Cov}_{F, \mathcal{T}}\left[\mathbb{E}_{i}[D], \frac{d X_{1 i}}{d \tau}\right]}_{\text {Belief dispersion }}+\underbrace{\left(\mathbb{E}[D]-\mathbb{E}_{F, \mathcal{T}}\left[\mathbb{E}_{i}[D]\right]\right) \frac{d S_{1 k}}{d \tau}}_{\begin{array}{c}
\text { Aggregate distortion } \times \\
\text { Investment response }
\end{array}}],
$$

where $\mathbb{E}_{F, \mathcal{T}}\left[\mathbb{E}_{i}[D]\right]$ denotes the average belief in the population of active investors, $\operatorname{Cov}_{F, \mathcal{T}}\left[\mathbb{E}_{i}[D], \frac{d X_{1 i}}{d \tau}\right]$ denotes a cross-sectional covariance and $\zeta(\tau) \equiv \int_{i \in \mathcal{T}} d F(i)$ is the fraction of active investors. Note that 
the investment response $\frac{d S_{1 k}}{d \tau}$ inherits the sign of $\frac{d P_{1}^{d}}{d \tau}-$ so when prices are higher, producers invest more and viceversa. Because the second term in (34) is in general non-zero when $\tau=0$, production generates a new first-order effect on welfare.

Intuitively, the optimal tax corrects two distortions induced by belief disagreement. First, given an amount of aggregate risk, the optimal tax seeks to reduce the dispersion in asset holdings induced by disagreement - some investors are holding too much risk and some others too little risk. This force is also present in exchange economies. Second, as long as the average belief is incorrect, the level of investment and production in the economy is too high - when investors are on average optimistic or too low. This provides a second rationale for taxation. Intuitively, the agents in the economy are holding too much aggregate risk when they are optimistic or too little when they are pessimistic.

Note that asset prices play no direct role on the determination of the optimal tax, even in a model with production. All welfare losses must be traced back to distortions in "quantities", either in portfolio allocations - captured by $\frac{d X_{1 i}}{d \tau}$ - or in production decisions - captured by $\frac{d S_{1 k}}{d \tau}$.

It can be shown - see appendix A - that the optimal tax can be written as a linear combination between the optimal tax for the equivalent exchange economy and the optimal tax in a (fictitious) production economy with a single investor with belief $\mathbb{E}_{F, \mathcal{T}}\left[\mathbb{E}_{i}[D]\right]$, that is:

$$
\begin{gathered}
\tau^{*}=\omega \tau_{\text {exchange }}^{*}+(1-\omega) \zeta(\tau) \tau_{\text {production' }}^{*} \\
\text { where } \tau_{\text {exchange }}^{*}=\frac{\operatorname{Cov}_{F, \mathcal{T}}\left[\frac{\mathbb{E}_{i}[D]}{P_{1}}, \frac{d X_{1 i}}{d \tau}\right]}{2 \int_{i \in \mathcal{B}, \mathcal{T}} \frac{d X_{1 i}}{d \tau} d F(i)} \text { and } \tau_{\text {production }}^{*}=\frac{\mathbb{E}[D]-\mathbb{E}_{F, \mathcal{T}}\left[\mathbb{E}_{i}[D]\right]}{P_{1}}
\end{gathered}
$$

And the weight $\omega$, which can take any real value, is defined as $\omega \equiv \frac{2 \zeta(\tau) \int_{i \in \mathcal{B}, \tau} \frac{d X_{1 i} d F(i)}{d \tau} d F(\tau) \int_{i \in \mathcal{B}, \tau} \frac{d X_{1 i}}{d \tau} d F(i)+\left(-\frac{d S_{1 k}}{d \tau}\right)}{\text {. The }}$ term $\zeta(\tau)$ corrects for the size of investors who marginally hold aggregate risk. Assumption [OBPS] still determines the sign of $\tau_{\text {exchange, }}^{*}$ but it is not sufficient anymore to pin down the sign of the optimal tax. The sign of $\tau_{\text {production }}^{*}$ is positive when $\mathbb{E}[D]-\mathbb{E}_{F}\left[\mathbb{E}_{i}[D]\right]$ and $\frac{d S_{1 k}}{d \tau}$ have the same sign and negative when they have opposite signs.

Sign of $\tau_{\text {production }}^{*}$ To provide further intuition for the sign of $\tau_{\text {production, }}^{*}$ assume that all investors have the same risk aversion coefficient $A_{i}=A$ and that $\gamma_{1}=0$. These assumptions imply that, in equilibrium, $\frac{d S_{1 k}}{d \tau}=\gamma_{2} \frac{d P_{1}}{d \tau}$. By defining $\hat{P}_{1}$ as the equilibrium price in a fictitious economy in which all investors hold correct beliefs, under the (strong) assumption that every buyer (seller) remains a buyer (seller) and that the fraction of inactive investors is constant, we can write:

$$
\mathbb{E}[D]-\mathbb{E}_{F, \mathcal{T}}\left[\mathbb{E}_{i}[D]\right]=v\left(\hat{P}_{1}-P_{1}\right),
$$

where $v>0$ is defined in appendix A. Intuitively, when investors are optimistic on aggregate, the equilibrium price is higher and viceversa. This exact mapping between aggregate belief distortions and prices is intuitive, but it relies on strong conditions. This is the case because the term $\mathbb{E}[D]-\mathbb{E}_{F}\left[\mathbb{E}_{i}[D]\right]$ determines welfare directly but it only affects prices indirectly. 
We can thus express the sign of $\tau_{\text {production }}^{*}$ as:

$$
\operatorname{sgn}\left(\tau_{\text {production }}^{*}\right)=\operatorname{sgn}\left(\left(\hat{P}_{1}-P_{1}\right) \frac{d P_{1}}{d \tau}\right)
$$

Therefore, as long as a marginal tax increase stabilizes prices at the margin, that is, it reduces prices, $\frac{d P_{1}}{d \tau}<0$, when prices are too high with respect to the correct price $\hat{P}_{1}$ and viceversa, the optimal tax is positive. Is this is a plausible scenario? As shown in lemma 1 in page 12, when risk aversion is identical among investors, $\frac{d P}{d \tau}$ is negative when the number of buyers is larger than the number of sellers and viceversa. Hence, the relation between the average belief distortion and the pattern of trading

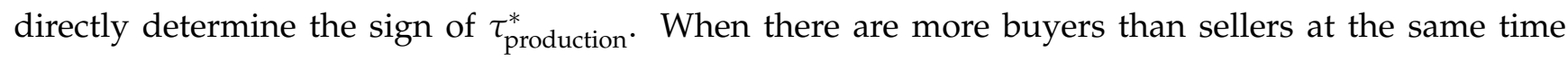
that the average belief among investors is larger than the planner's belief, the optimal production tax is positive. Taking as starting point a situation with unbiased investors and equal number of buyers and

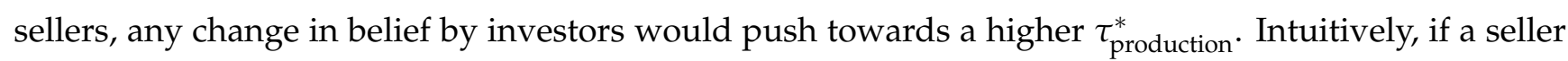
becomes sufficiently optimistic and becomes a buyer — provided that other investors do not change their positions at the margin - , this would generate a negative term $\mathbb{E}[D]-\mathbb{E}_{F, \mathcal{T}}\left[\mathbb{E}_{i}[D]\right]$, because investors are on average more optimistic, and a negative derivative $\frac{d P_{1}}{d \tau}$, because there are more buyers in equilibrium. The opposite would occur if a buyer becomes pessimist. However, in general, there are many other configurations of average beliefs with buying and selling patterns that may push towards a negative $\tau_{\text {production }}^{*}$

Finally, note that proposition 12 extends to more sophisticated capital structure theories, as long as heterogeneous beliefs determine production through security prices. Intuitively, the envelope theorem would apply to every individual decision. This logic remains true even if the investment side of the economy faces frictions either in funding or production, as long as the planner is also constrained to face them.

\subsection{Hayekian production/Information diffusion}

In this section, some investors have access to better information about payoffs than others. When informed investors trade, their information is incorporated into the asset price, helping managers to increase efficiency by adjusting production decisions. ${ }^{42}$ The notion that financial markets aggregate and convey information useful for production goes back to Hayek (1945).

The model of this section has been simplified in many dimensions. My single goal is to show, in the simplest model with information diffusion in a production economy, that a transaction tax creates a new first-order distortion. Further research should clarify how different assumptions about information and learning modify these results. ${ }^{43}$

\footnotetext{
${ }^{42}$ Most models of trading with dispersed information do not include production. Some papers that do model the link from stock prices to production decisions are Leland (1992), Dow and Gorton (1997), Subrahmanyam and Titman (1999), Goldstein and Guembel (2008), Ozdenoren and Yuan (2008), Angeletos, Lorenzoni and Pavan (2010), Albagli, Hellwig and Tsyvinski (2011) and Goldstein, Ozdenoren and Yuan (2013). None of them analyze the effect of transaction taxes.

${ }^{43}$ For instance, this model only features a single informed investor, but it is important to understand how transaction taxes affect information aggregation when it is dispersed among many investors. It is also important to understand how taxes
} 
Assumptions Time is discrete and the economy lasts two periods $t=\{1,2\}$. There are three different types of agents: informed investors, uninformed investors and a firm manager. The measure of informed investors is given by $\pi_{I}$ and the measure of uninformed investors is given by $\pi_{U}=1-\pi_{I}$; I use the index $i=\{I, U\}$ to denote an investor of either type. Investors maximize CARA utility subject to the same budget constraint as in the baseline model - see equation (1) in page 6.

The single policy instrument available to the planner is again a single linear financial transaction tax. I rule out any policy that involves transferring information among agents. For simplicity, I assume that all investors hold initially the same number of shares $X_{0 i}=Q$ of the risky asset and that their hedging needs are zero, that is, $\operatorname{Cov}\left[E_{2 i}, D\right]=0, \forall i$.

The actions of the firm manager determine endogenously the payoff of the risky asset. He chooses the scale of production $k^{*}$ with the goal of maximizing the profit per share $\Pi$, which in this economy has three components and is given by:

$$
\Pi=D+\theta+\delta\left(\theta^{2}-\left(\theta-k^{*}\right)^{2}\right), \quad \delta \geq 0
$$

The sum $D+\theta$ captures the payoff of the risky asset which is not determined by production. The first term $D$ is normally distributed with mean $\mathbb{E}[D]$ and variance $\mathbb{V}$ ar $[D]$. The second term $\theta$ is normally distributed with zero mean and variance $\operatorname{Var}[\theta]$. The last term $\delta\left(\theta^{2}-\left(\theta-k^{*}\right)^{2}\right)$ captures the role of production in this economy. The firm manager must choose the scale of investment $k^{*}$ close to the fundamental $\theta$; any deviation would decrease flow profits for the firm. Intuitively, when a firm has a high (low) exogenous productivity $\theta$ it must increase (reduce) its scale $k^{*}$ in order to produce efficiently.

The functional form for $\Pi$ is chosen for tractability. When $\operatorname{Var}[\theta]=0$ and $\delta=0$ this formulation becomes a special case of the baseline model.

Informed investors learn the value of the actual realization of $\theta$, but uninformed investors do not. Moreover, I assume that uninformed investors are (technologically) unable to update their beliefs from the price. They put no weight on the information conveyed through prices by the informed investors because of a behavioral bias. This assumption allows investors to trade because they "agree to disagree". This motive for trading is analogous to the one due to heterogeneous priors/beliefs discussed in the previous sections of the paper. For simplicity, all investors hold correct beliefs about $D .{ }^{44}$

The firm manager knows the structure of the economy and can extract information about $\theta$ from the equilibrium price $P_{1}$. Hence, he solves the following maximization problem to determine $k^{*}$ :

$$
k^{*}=\arg \max _{k} \mathbb{E}\left[D+\theta+\delta\left(\theta^{2}-(\theta-k)^{2}\right) \mid P_{1}\right]
$$

The problem of the firm is convex in $k$, so the first order condition is necessary and sufficient to characterize the optimal investment choice:

$$
k^{*}=\mathbb{E}\left[\theta \mid P_{1}\right]
$$

modify welfare when the equilibrium is not fully revealing. The existence of inaction regions creates important technical challenges, greatly complicating the answers to both questions.

${ }^{44}$ Allowing some, but not all, uninformed investors to update their beliefs from prices does not modify the results. It is also straightforward to introduce further belief disagreement among investors by assuming that they have heterogeneous priors about $\mathbb{E}[D]$. 
When the firm manager does not receive any information through the asset price, he sets $k^{*}$ according his prior belief about $\theta$, that is $k^{*}=\mathbb{E}[\theta]=0$. When informed investors decide to trade, the manager can perfectly infer $\theta$ from observing the equilibrium price, so he sets $k^{*}=\theta$.

From now on, I impose the following assumption.

Assumption 5. (Effects of trading on production not internalized) Informed investors do not internalize the effect of their trading activity on production. Therefore, both informed and uninformed investors assume that the firm manager sets $k^{*}$ according to the unconditional expectation of $\theta$, that is, they perceive that $k^{*}=\mathbb{E}[\theta]=0$.

Since I have already assumed that informed investors lack the technology to infer information from the asset price, assumption 5 is not restrictive for them. However, it is important from the perspective of informed investors, which could in principle take into account the effect of their trading into production. From a conceptual standpoint, this assumption can be motivated by arguing that information is truly dispersed, so one investor at a time is not sufficient to release useful information to guide production, so many must trade to have any impact on prices. From a technical standpoint, the fact that informed investors are not aware of how their trading activity affects production keeps their individual problem concave. ${ }^{45}$ I will be explicit below about how this assumption affects welfare and how the optimal tax varies when it is relaxed.

Given the assumptions, the equilibrium of this economy is fully revealing from the perspective of producers. Hence, the definition of equilibrium is standard for every realization of the signal received by informed investors - see appendix A for a full definition. Because $\theta$ can take large negative values, I must deal explicitly with the possibility that $P_{1}$ may be negative.

Results: equilibrium characterization The optimal portfolio choice of every investor can be found by solving:

$$
\max _{X_{1 i}}\left\{\left(\mathbb{E}[D]+\mathbb{E}_{i}[\theta]-P_{1}\right) X_{1 i}-\frac{A_{i}}{2} \mathbb{V a r}_{i}[D+\theta]\left(X_{1 i}\right)^{2}-\left|\Delta X_{1 i}\right|\left|P_{1}\right| \tau\right\}
$$

Note that $\mathbb{E}_{i}[\theta]$ equals 0 for uninformed investors but it equals $\theta$ for informed ones and $\operatorname{Var}_{i}[D+\theta]$ equals $\mathbb{V a r}[D]+\mathbb{V a r}[\theta]$ for uninformed investors but it equals $\mathbb{V} a r[D]$ for informed investors. In the trade region, the portfolio demand of a given investor $i$ is given by:

$$
X_{1 i}=\frac{\mathbb{E}[D]+\mathbb{E}_{i}[\theta]-P_{1}\left(1+\operatorname{sgn}\left(\Delta X_{1 i}\right) \operatorname{sgn}\left(P_{1}\right) \tau\right)}{A_{i} \mathbb{V} a r_{i}[D+\theta]} \quad \text { for } i=\{I, U\}
$$

Uninformed investors trade less aggressively because they perceive that the risky asset has a larger variance, due to their worse information. The portfolio demand in this section only differs from the one in the baseline model in that $\tau$ now multiplies $\operatorname{sgn}\left(P_{1}\right)$ instead of $P_{1}$, to account for negative prices.

Given the optimal portfolio holdings and the market clearing condition $\pi_{I} X_{1 I}+\pi_{U} X_{1 U}=Q$, the equilibrium price of the risky asset can be written as:

$$
P_{1}=\frac{\pi_{U} \frac{\mathbb{E}[D]}{A_{U} \mathbb{V} a r[D+\theta]}+\pi_{I} \frac{\mathbb{E}[D]+\theta}{A_{I} \mathbb{V a r}[D]}-Q}{\pi_{U} \frac{1+\operatorname{sgn}\left(\Delta X_{1 U}\right) \operatorname{sgn}\left(P_{1}\right) \tau}{A_{U} \mathbb{V} \operatorname{ar}[D+\theta]}+\pi_{I} \frac{1+\operatorname{sgn}\left(\Delta X_{1 I}\right) \operatorname{sgn}\left(P_{1}\right) \tau}{A_{I} \mathbb{V a r}[D]}}
$$

\footnotetext{
${ }^{45}$ If investors internalize their effect on production, a non-convexity arises, greatly complicating the analysis. Intuitively, even around the optimum, there is a "zero order" gain from trading but only a first-order loss from facing the linear tax.
} 
The interpretation of (36) is identical to the baseline model. For tractability, I impose the following restrictions.

Assumption 6. (Assumptions on parameters) The parameters of the model are such that:

a) $\pi_{I}=\pi_{U}=\frac{1}{2}$ and $A_{U} \mathbb{V}$ ar $[D+\theta]=A_{I} \mathbb{V}$ ar $[D]$.

b) The value of $\mathbb{E}[D]$ is sufficiently large so that $\mathbb{E}[D]-Q A_{I} \mathbb{V}$ ar $[D]>0$.

Assumption 6a implies that the equilibrium price $P_{1}=\mathbb{E}[D]+\frac{\theta}{2}-Q A_{I} \mathbb{V}$ ar $[D]$ is independent of $\tau$. Assumption $6 \mathrm{~b}$ guarantees that the equilibrium price is positive at the trade boundaries. Both set of restrictions simplify the characterization of the inaction region, but entail little loss of generality.

Lemma 3 characterizes the inaction region, which is crucial for the normative analysis.

Lemma 3. (Inaction thresholds) a) There is no-trade in equilibrium for values of $\theta$ such that:

$$
\underline{\theta}(\tau) \leq \theta \leq \bar{\theta}(\tau)
$$

where the thresholds, $\underline{\theta}(\tau)$ and $\bar{\theta}(\tau)$, which are functions of $\tau$, are given by:

$$
\underline{\theta}(\tau)=-\frac{1}{1+\tau} 2\left(\mathbb{E}[D]-Q A_{I} \mathbb{V} a r[D]\right) \quad \bar{\theta}(\tau)=\frac{1}{1-\tau} 2\left(\mathbb{E}[D]-Q A_{I} \mathbb{V} a r[D]\right)
$$

b) The no-trade region increases with the size of the tax. The derivatives of both thresholds are strictly different from zero, even when $\tau=0$. Both statements are summarized in:

$$
\left.\frac{d \underline{\theta}(\tau)}{d \tau}\right|_{\tau \in[0, \infty]}<0 \quad \text { and }\left.\quad \frac{d \bar{\theta}(\tau)}{d \tau}\right|_{\tau \in[0, \infty]}>0
$$

Proof. See appendix A.

Figure 3 graphically shows the inaction region as a function of the realization of $\theta$.

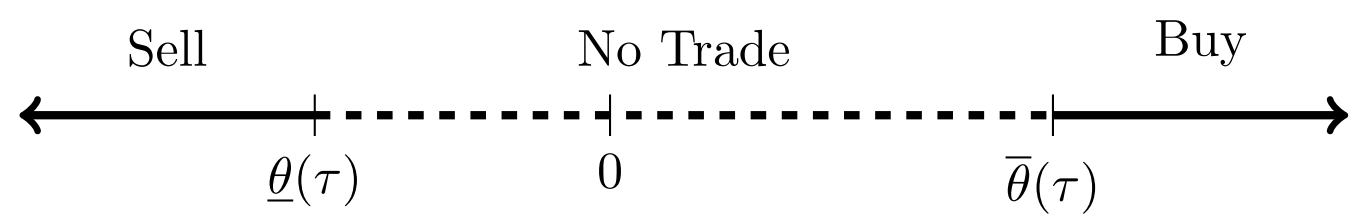

Figure 3: No-trade region

From an informed investor perspective, when his signal is sufficiently close to the prior $\theta=0$, the marginal gains from trading are smaller than the marginal cost of the tax, so it is optimal not to trade. As expected, in the limit when the optimal tax tends to zero, the no-trade region disappears. The fact that the derivatives of the no-trade thresholds are non-zero even when $\tau=0$ it is important for the welfare analysis, since this implies that the welfare loss from taxation is first-order.

Lastly, the fact that trading completely stops when the informed investor decides not to trade is an artifact of assuming only two types of investors; it is straightforward to allow for additional investors who trade for other fundamental reasons. 
Results: welfare analysis Because in this section the dispersion in investors' beliefs may be due to different information, it is important to refine the criterion used to calculate social welfare. I assume that the planner calculates indirect utility for every investor respecting his individual information, but updating rationally. In this model, this implies that the planner acts as if he observed $\theta$. This approach considers trading in private information as fundamental trading.

In a more general environment, the planner would calculate indirect utility under two premises. First, using a common prior. Second, updating rationally, although respecting informational constraints. This approach corrects behavioral distortions while respecting technological constraints. Finally, note that the planner, unlike the investors, takes into account that trading modifies the informational content of prices and how this determines production and welfare.

Under this criterion, social welfare - denoted by $V$ - can be written as the sum of the indirect utility for each investor $i$, under the planner's distribution, that is:

$$
V(\tau)=\sum_{i} \lambda_{i} \pi_{i} V_{i}
$$

where we can write individual welfare from the planner's perspective as:

$$
\begin{gathered}
V_{i} \equiv \mathbb{E}_{\theta}\left[\mathbb{E}\left[U_{i}\left(W_{2 i}\right) \mid \theta\right]\right]=\mathbb{E}_{\theta}\left[-e^{-A_{i} \hat{V}_{i}(\theta)}\right] \\
\text { where } \hat{V}_{i}(\theta)=(\underbrace{\mathbb{E}[D]+\theta+\delta\left(\theta^{2}-\left(\theta-k^{*}\right)^{2}\right)}_{\mathbb{E}[\Pi]}-P_{1}) X_{1 i}+P_{1} X_{0 i}-\frac{A_{i}}{2} \mathbb{V a r}[D] X_{1 i}^{2}
\end{gathered}
$$

And where the planner is aware that $k^{*}=0$ in no-trade regions and $k^{*}=\theta$ in the trade regions. The planner averages welfare over all the possible realizations of the fundamental $\theta$ and, according to the welfare criterion, uses $\mathbb{E}[D+\theta]=\mathbb{E}[D]+\theta$ and $\operatorname{Var}[D+\theta]=\mathbb{V} a r[D]$. I denote expectations over the realization of the fundamental $\theta$ by $\mathbb{E}_{\theta}[\cdot]$.

For a given investor $i$, the marginal change in welfare from the planner's perspective is given by:

$$
\frac{d V_{i}}{d \tau}=\mathbb{E}_{\theta}\left[A_{i} e^{-A_{i} \tilde{V}_{i}} \frac{d \tilde{V}_{i}}{d \tau}\right]=\mathbb{E}_{\theta}\left[\mathbb{E}\left[U^{\prime}\left(W_{2 i}\right)\right] \frac{d \tilde{V}_{i}}{d \tau}\right],
$$

where the derivative $\frac{d \tilde{V}_{i}}{d \tau}$ can be written as:

$$
\frac{d \tilde{V}_{i}}{d \tau}=\left(\mathbb{E}[\Pi]-r_{i} \mathbb{E}_{i}[\Pi]+P_{1} r_{i}\left(1+\operatorname{sgn}\left(\Delta X_{1 i}\right) \operatorname{sgn}\left(P_{1}\right) \tau\right)\right) \frac{d X_{1 i}}{d \tau}-\Delta X_{1 i} \frac{d P_{1}}{d \tau}
$$

This expression is similar to the one derived in section 5.4, in which investors perceive different variances. Note that $r_{U}=\frac{\operatorname{Var}[D]}{\operatorname{Var}[D+\theta]}<1$ for uninformed investors but $r_{I}=1$ for informed investors. Intuitively, the planner gives less weight to the distortion of the less responsive investor.

I introduce assumption [NR $\theta$ ] to leave aside again redistributional considerations.

Assumption 7. [NR $\theta$ ] (No redistribution) The product of the social welfare weight $\lambda_{i}$ with the expected marginal utility under the planner's probability distribution $\mathbb{E}\left[U_{i}^{\prime}\left(W_{2 i}\right)\right]$ does not vary across the distribution of investors for any $\theta$, that is, $\lambda_{i} \mathbb{E}\left[U_{i}^{\prime}\left(W_{2 i}\right)\right]$ is constant for all investors for every $\theta$. 
Under assumption [NR $\theta$ ], the marginal change in social welfare given $\theta$ is determined by:

$$
\left.\frac{d V}{d \tau}\right|_{\theta}=\sum_{i} \pi_{i}\left(-r_{i} \mathbb{E}_{i}[\Pi]+P_{1} r_{i}\left(1+\operatorname{sgn}\left(\Delta X_{1 i}\right) \operatorname{sgn}\left(P_{1}\right) \tau\right)\right) \frac{d X_{1 i}}{d \tau}
$$

Under assumption [NR $\theta$ ], the planner does not need to know the realization of $\theta$ when calculating $\left.\frac{d V}{d \tau}\right|_{\theta}$. Likewise, the impact of terms-of-trade effects washes out in the aggregate.

Before presenting the main propositions of this section, it is useful to derive the following result.

Lemma 4. (Welfare gap) a) Around the inaction thresholds, social welfare is strictly larger when there is trade than when there is no-trade. Formally $V^{T}(\theta)-V^{N T}(\theta)>0$, where $\theta=\{\underline{\theta}, \bar{\theta}\}$ and $V^{T}(\underline{\theta})$ and $V^{N T}(\underline{\theta})$ are given by $V^{T}(\underline{\theta}) \equiv \lim _{\theta \rightarrow \underline{\theta}^{-}} V(\theta)$ and $V^{N T}(\underline{\theta}) \equiv \lim _{\theta \rightarrow \underline{\theta}^{+}} V(\theta)$, while $V^{T}(\bar{\theta})$ and $V^{N T}(\bar{\theta})$ are equivalently defined but with opposite limits of integration — see appendix A. T and NT stand for trading and no-trading.

b) Under assumption [NR $\theta$ ], we can write:

$$
V^{T}(\theta)-V^{N T}(\theta)=\sum_{i} \pi_{i}\left(\frac{1}{A_{i}}\left(1-e^{-A_{i} \delta \theta^{2} X_{0 i}}\right)\right) \approx \delta \theta^{2} Q>0,
$$

where $\theta=\{\underline{\theta}, \bar{\theta}\}$ and the last term provides an approximation around $\delta=0$ or $A_{i}=0, \forall i$.

Proof. See appendix A.

Lemma 4a shows that there is a discontinuity in social welfare at the inaction boundaries. Intuitively, because the planner internalizes the effect of trading in production, he acknowledges that production efficiency decreases when informed investors stop trading.

The value of $V^{T}(\theta)-V^{N T}(\theta)$ is given by the sum of the difference in welfare between regions of both informed and uninformed agents. This difference is strictly positive for informed investors in this model because of assumption 5, which I have imposed for convenience. In a model in which investors internalize the effects of their individual trading in production, an envelope condition would imply that this term is zero. However, there is no reason to have an envelope condition for uninformed investors: this is the source of the informational externality. Intuitively, informed investors do not internalize the effect of their trades on the welfare of other investors through increased production efficiency. Therefore, the equivalent to lemma 4 would hold even without assumption 5.

The approximation in lemma $4 \mathrm{~b}$ is intuitive. The wedge between trading and no trading at the margin is increasing in the product of the benefit of information production per share $\theta^{2}$ times the supply of asset shares $Q$.

Proposition 13. (Marginal effect on welfare with information diffusion) a) Under assumption [NR $\theta$ ], the marginal change in social welfare from varying the financial transaction tax $\tau$ is given by:

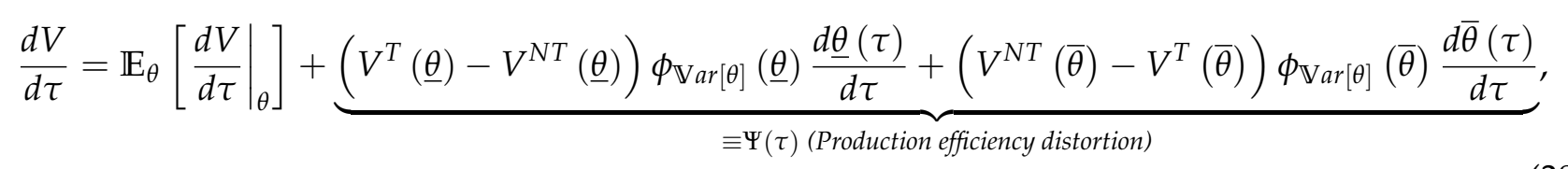

where $\left.\frac{d V}{d \tau}\right|_{\theta}$ is given by (37), $V^{T}(\theta)-V^{N T}(\theta)$ denotes the difference at $\theta$ in social welfare between trading and no trading and $\phi_{\mathbb{V} a r[\theta]}(\cdot)$ denotes the $p d f$ of a normal distribution with zero mean and variance $\mathbb{V} a r[\theta]$. 
b) When $\tau=0$, the marginal change in social welfare associated to the diffusion of information is strictly negative, that is:

$$
\left.\Psi(\tau)\right|_{\tau=0}<0
$$

where $\Psi(\tau)$ is defined in (38).

Proof. See appendix A.

The first term in (38) is identical to the one in the baseline model, although now as an average across realizations of $\theta$. Note that, in the no-trade region, $\left.\frac{d V}{d \tau}\right|_{\theta}=0$. The second term in (38), defined as $\Psi(\tau)$, captures the welfare loss generated by a marginal tax increase due to a reduction in production efficiency. Intuitively, when informed investors decide not to trade, production efficiency is lower because the firm manager has less information in those states.

Using the results of lemmas 3 and 4 and the fact that $\phi_{\mathbb{V a r}[\theta]}(\cdot)$ is strictly positive, we can immediately conclude that $\Psi(\tau)$ is strictly negative for any $\tau$, even when $\tau=0$, as shown in proposition $13 \mathrm{~b}$. This shows that the welfare loss generated by a transaction tax due to a reduction in information diffusion is first-order. Intuitively, an infinitesimal change in the size of the tax starting from $\tau=0$ creates an inaction region of strictly positive measure. This fact, combined with the pre-existing wedge in social welfare, generates the local first-order loss. In the baseline model (when $\delta=0$ ), there is no wedge in social welfare between trading and no trading regions, so $V^{T}(\cdot)$ always equal $V^{N T}(\cdot)$ and $\Psi(\tau)=0$, for any value of $\tau$.

Finally, I derive the optimal transaction tax.

Proposition 14. (Optimal tax with information diffusion) Under assumption [NR $\theta]$, using the approximation from lemma 4 , the optimal tax can be written as:

$$
\tau^{*}=\frac{\mathbb{E}_{\theta}\left[\sum_{i} \pi_{i} r_{i} \mathbb{E}_{i}[D] \frac{d X_{1 i}}{d \tau}\right]+\delta Q\left[(\underline{\theta})^{2} \phi_{\mathbb{V} a r[\theta]}(\underline{\theta}) \frac{d \theta}{d \tau}+(\bar{\theta})^{2} \phi_{\mathbb{V a r}[\theta]}(\bar{\theta}) \frac{d \bar{\theta}}{d \tau}\right]}{P_{1} \mathbb{E}_{\theta}\left[\sum_{i} \pi_{i} r_{i}\left(1+\operatorname{sgn}\left(\Delta X_{1 i}\right) \operatorname{sgn}\left(P_{1}\right) \frac{d X_{1 i}}{d \tau}\right)\right]}
$$

Proof. See appendix A.

Intuitively, the optimal tax can be written as the sum of the tax in a exchange economy plus a corrective term - which, in this case, favors a lower tax or a subsidy, since it is always negative. Therefore, we can additively decompose the optimal tax in the following way:

$$
\tau^{*}=\tau_{\text {Exchange }}^{*}+\tau_{\text {Information' }}^{*}
$$

where $\tau_{\text {Exchange }}^{*}$ and $\tau_{\text {Information }}^{*}$ are given in appendix A. Note that equilibrium portfolio tax sensitivities do not play the role of assigning relative weights to the distortions - this shows that the nature of information distortions is different from (Walrasian) distortions in portfolio allocations or production.

Because $\tau_{\text {Information }}^{*}<0$, the optimal tax is always lower when information diffusion plays a role in determining production. Importantly, if there is no belief disagreement, so $\tau_{\text {Exchange }}^{*}=0$, the optimal policy calls for a trading subsidy, not for the laissez-faire situation with $\tau^{*}=0$. It may thus be the case that a zero tax optimally balances the gain from eliminating non-fundamental trades with the loss induced by preventing information diffusion. 


\section{Endogenous information acquisition}

To keep the paper focused, I have opted for not including results on endogenous information acquisition. It is nonetheless possible to add an ex-ante stage to the model of this section in which informed investors decide whether to acquire information, at a cost, or not. As in the exogenous information case, the presence of informational externalities implies that any tax creates an additional first-order effect on welfare. However, in theory, this new mechanism can shift the optimal tax in any direction. This is not surprising, given the wide range of results found in the literature on endogenous information acquisition. ${ }^{46}$ The results of this paper can be seen as an approximation to the situation in which the endogenous choice of information is not sensitive to transaction taxes.

\section{Conclusion}

\section{Empirical counterparts to optimal tax formulas}

Most of the optimal tax formulas derived in this paper only depend on a few sufficient statistics. ${ }^{47}$ In particular, under natural conditions, the distribution of expected returns across investors turns out to be the single sufficient statistic for the optimal tax, independently of many other features of the environment. Looking forward, it would be helpful to have better empirical knowledge of the distribution of expected returns. Both the revealed preference approach and the survey approach have the potential to shed light on this issue. ${ }^{48}$

\section{Final remarks}

This paper has provided a conceptual framework to understand the welfare implications of financial transaction taxes in an equilibrium model of competitive financial markets. While transaction taxes distort all trades, I have shown that they have different welfare implications for fundamental versus non-fundamental trades.

The main conclusions of this paper are the following. First, the existence of trading among investors

\footnotetext{
${ }^{46}$ The idea that investors do not acquire sufficient information because they fail to internalize the benefits to others goes back to Grossman and Stiglitz (1980). On the one hand, acquiring information is positive to increase production efficiency, as in this section. On the other hand, it may generate adverse selection. Dang, Gorton and Holmstrom (2009) emphasize the welfare benefits of not acquiring information. Moreover, it is not obvious how behavioral biases, which generate non-fundamental trading, interact with the endogenous choice of information. For instance, overconfident investors may at the same time be trading too much because they agree to disagree - as emphasized in the baseline model — at the same that they acquire too much information - improving production efficiency. There is scope to analyze in greater detail how transaction taxes interact with these issues.

${ }^{47}$ See Chetty (2009) for a forceful defense of the sufficient statistic approach. See also Hendren (2013), whose policy elasticity approach directly fits this paper.

${ }^{48}$ Using survey methods, Amromin and Sharpe (2012) and Greenwood and Shleifer (2013) take a first step in that direction; see also Ben-David, Graham and Harvey (2013). Banerjee (2011) uses a revealed preference approach in a related environment. To come up with a meaningful value for $\tau^{*}$, more and better data about expectations, and ideally portfolio decisions, would be needed.
} 
due to dispersion in beliefs creates a powerful force in favor of a strictly positive tax. The optimal tax can be expressed as a function of the distribution of beliefs and the sensitivities of portfolio allocations with respect to tax changes. The optimal tax accounts for the fact that trading at higher frequencies is particularly discouraged. The frequency of trading affects the magnitude of the optimal tax but the pattern of belief distortions is what determines its sign. Second, as long as investors' expectations are incorrect on average and financial markets determine investment and production, there exists an additional argument in favor of a higher or lower optimal tax. Through this mechanism, higher taxes are for instance optimal when investors are too optimistic on average at the same time that the demand side of the market is more price elastic than the supply side. Third, when information diffusion is key to improving production efficiency, the optimal policy calls for a transaction subsidy, to correct for a learning externality. The optimal tax should balance all three considerations.

Hence, this paper has shown that the current status quo in most financial exchanges - a zero transaction tax - is only optimal in knife-edge situations from a theoretical viewpoint. Whether legal and practical concerns regarding tax avoidance, which have not been considered in this paper, are sufficiently important to advise against implementing any tax (or subsidy) on trading in financial markets remains an open question. 


\section{Appendix: Examples}

\section{Example 1: Only optimists and pessimists/No fundamental trading}
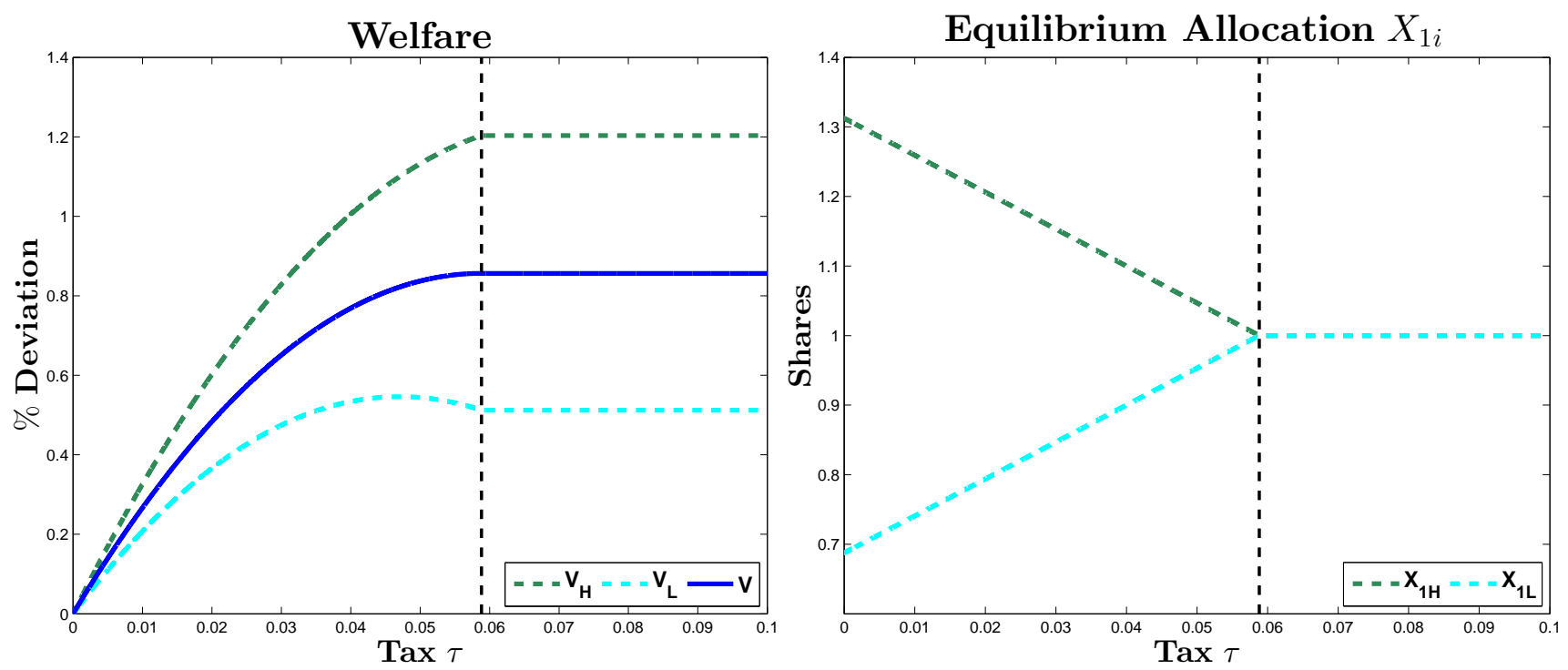

Equilibrium Price $P_{1}$
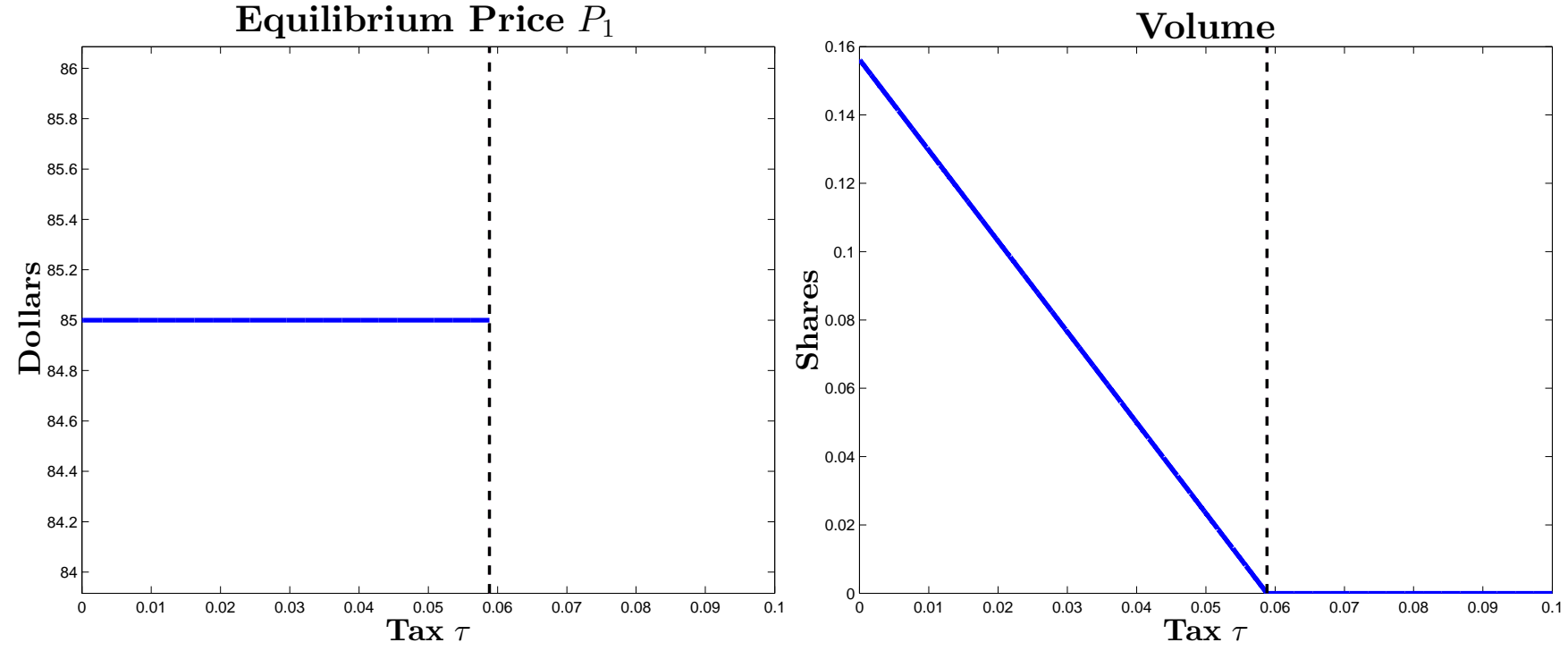

Figure 4: Example 1

The top left plot shows the indirect utility for each investor from the planner's perspective $V_{i}$ and social welfare $V$. The bottom left one shows the equilibrium price. The top right plot shows the equilibrium levels of trade for each investor and the bottom right one shows the total amount of trade in equilibrium, given by $\int_{i \in \mathcal{B}} X_{1 i} d F(i)$. The vertical purple dashed lines represent tax levels at which at least one of the investors stops trading. The vertical black dashed line represents the optimal tax level. 


\section{Example 2: Optimists, pessimists and correct investors}
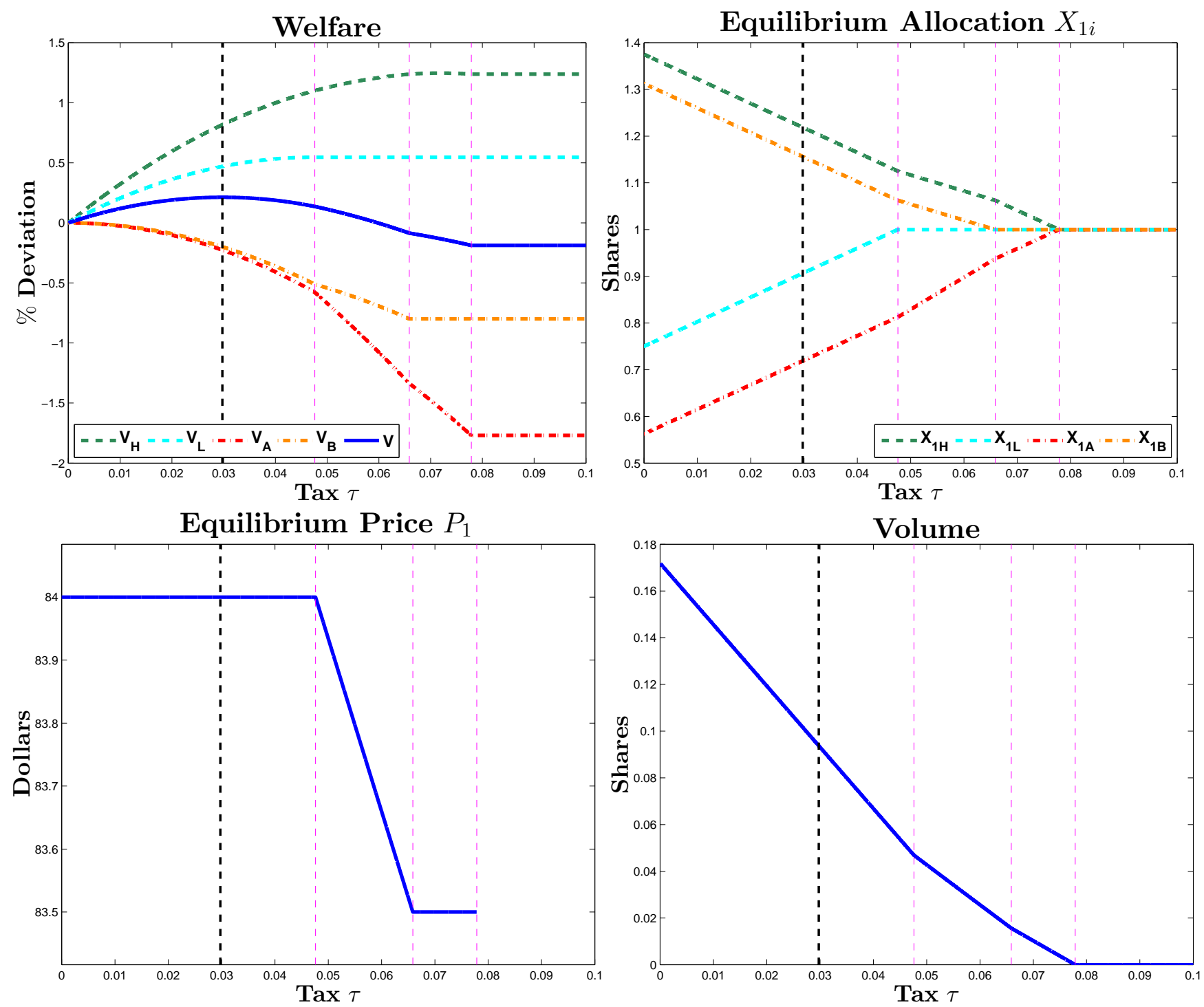

Figure 5: Example 2

The top left plot shows the indirect utility for each investor from the planner's perspective $V_{i}$ and social welfare $V$. The bottom left one shows the equilibrium price. The top right plot shows the equilibrium levels of trade for each investor and the bottom right one shows the total amount of trade in equilibrium, given by $\int_{i \in \mathcal{B}} X_{1 i} d F(i)$. The vertical purple dashed lines represent tax levels at which at least one of the investors stops trading. The vertical black dashed line represents the optimal tax level. 


\section{Example 3: Pessimists buyers}
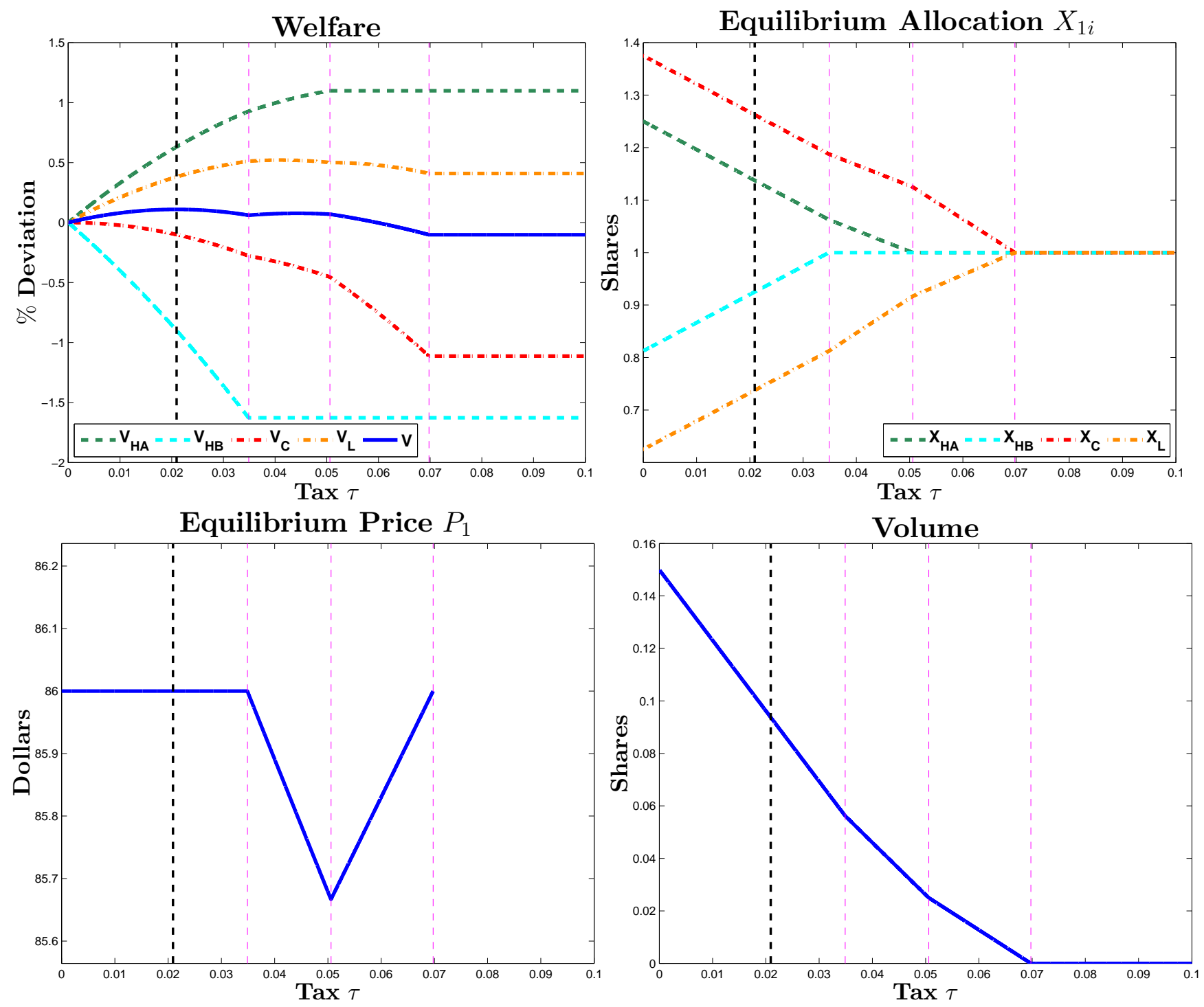

Figure 6: Example 3

The top left plot shows the indirect utility for each investor from the planner's perspective $V_{i}$ and social welfare $V$. The bottom left one shows the equilibrium price. The top right plot shows the equilibrium levels of trade for each investor and the bottom right one shows the total amount of trade in equilibrium, given by $\int_{i \in \mathcal{B}} X_{1 i} d F(i)$. The vertical purple dashed lines represent tax levels at which at least one of the investors stops trading. The vertical black dashed line represents the optimal tax level. 


\section{Example 4: Pre-existing trading costs}
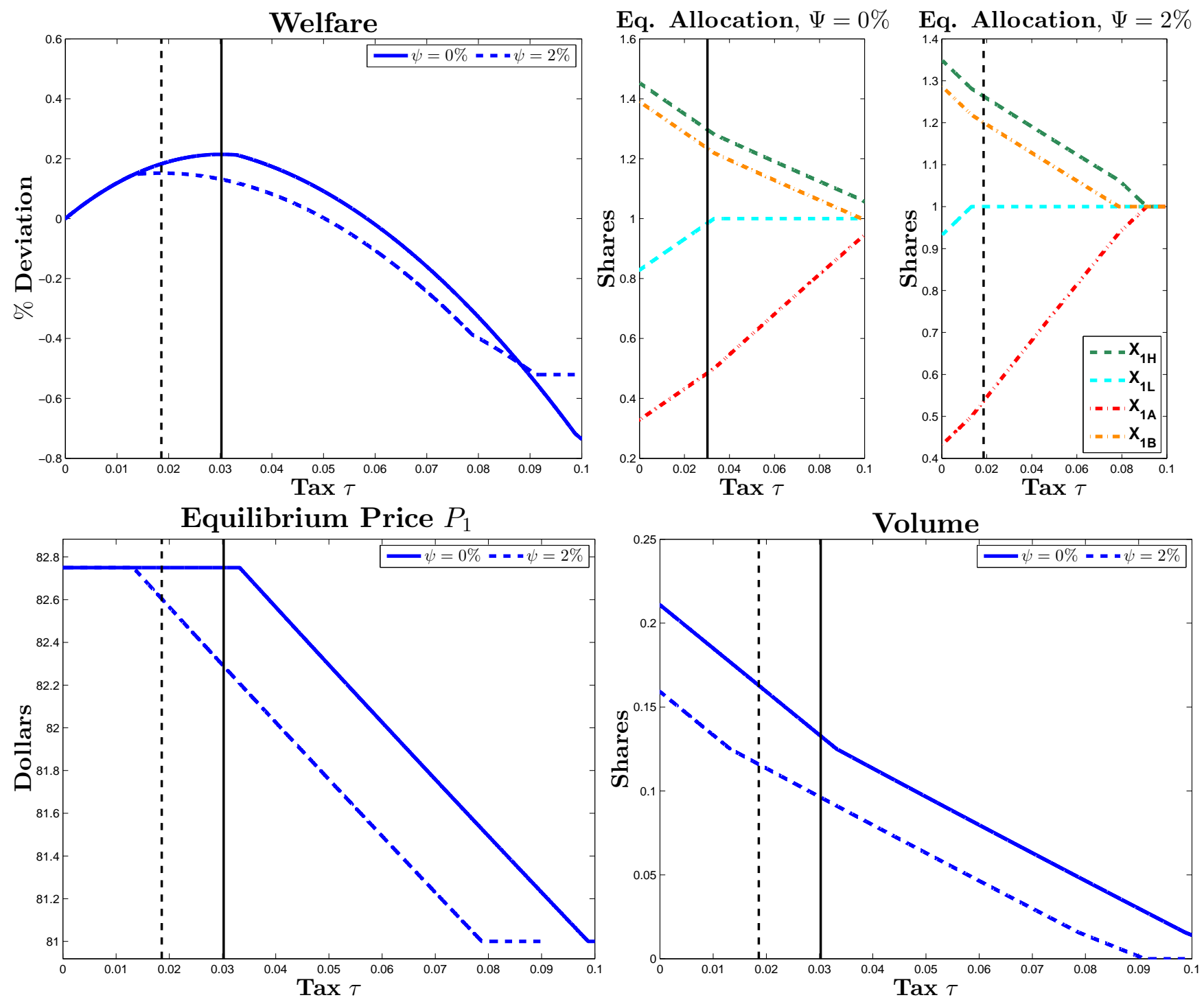

Figure 7: Example 4 - Transaction costs

The top left plot shows social welfare $V$ for both values of $\psi$. It is expressed in relative terms with respect to the $\tau=0$ case. The bottom left one shows the equilibrium price. The top right plot shows the equilibrium levels of trade for each investor and the bottom right one shows the total amount of trade in equilibrium, given by $\int_{i \in \mathcal{B}} X_{1 i} d F(i)$. With the exception of the equilibrium allocation plots, the dashed lines represent the $\psi=2 \%$ case, while the solid lines represent the $\psi=0 \%$ case. The vertical dashed lines denote the optimal tax for the $\psi=2 \%$ case, while the vertical solid lines denote the optimal tax for the $\psi=0 \%$ case. 


\section{Appendix A: Proofs and derivations}

\section{Proofs: Section 2}

\section{Assumption 1. [PP] (Positive prices)}

The equilibrium price is given by equation (7) in the paper. To guarantee that $P_{1}>0$, we only need to show that $\int_{i \in \mathcal{T}}\left(\frac{\mathbb{E}_{i}[D]-A_{i} \operatorname{Cov}\left[E_{2 i}, D\right]}{A_{i}}-X_{0 i} \mathbb{V a r}[D]\right) d F(i)$ is strictly positive, since $\int_{i \in \mathcal{T}} \frac{1+\operatorname{sgn}\left(X_{1 i}-X_{0 i}\right) \tau}{A_{i}} d F(i)$ is always strictly positive, given the restriction that $-1<\tau<1$. Equation (4) directly implies that $\frac{\mathbb{E}_{i}[D]-A_{i} \operatorname{Cov}\left[E_{2 i}, D\right]}{A_{i}}-X_{0 i} \mathbb{V} \operatorname{ar}[D]>0, \forall i$, because $X_{0 i} \leq Q$. This is sufficient to show that, under assumption 1 , the equilibrium price is strictly positive.

\section{Lemma 1. (Effect of $\tau$ on prices)}

a) Substituting (8) into the derivative of the budget constraint $\int \frac{d X_{1 i}}{d \tau} d F(i)=0$, we can find that $\frac{d P}{d \tau}=-\frac{\int_{i \in \mathcal{T}} \frac{\partial x_{1 i}}{\partial \tau} d F(i)}{\int_{i \in \mathcal{T}} \frac{\partial x_{1 i}}{\partial P} d F(i)}$. Note that this derivation takes into account the fact that a marginal tax change modifies the extensive margin choice of some investors, who decide whether to trade or not; these effects do not affect price changes because the net demand of an investor indifferent at the extensive margin is zero - this follows directly by using Leibniz rule. Taking derivatives directly on (5), we can also find that $\frac{\partial X_{1 i}}{\partial \tau}=\frac{-P_{1} \operatorname{sgn}\left(\Delta X_{1 i}\right)}{A_{i} \mathbb{V a r}[D]}$ and $\frac{\partial X_{1 i}}{\partial P}=\frac{-\left(1+\operatorname{sgn}\left(\Delta X_{1 i}\right) \tau\right)}{A_{i} \mathbb{V a r}[D]}$. Therefore, we have that $\frac{d P}{d \tau}=$ $-\frac{P_{1} \int_{i \in \mathcal{T}} \frac{\operatorname{sgn}\left(\Delta X_{1 i}\right)}{A_{i}} d F(i)}{\int_{i \in \mathcal{T}} \frac{1+\operatorname{sgn}\left(\Delta X_{1 i}\right)}{A_{i}} d F(i)}$. The sign of this expression is ambiguous. It depends on $\int_{i \in \mathcal{T}} \frac{\operatorname{sgn}\left(\Delta X_{1 i}\right)}{A_{i}} d F(i)$ : when $\int_{i \in \mathcal{T}} \frac{\operatorname{sgn}\left(\Delta X_{1 i}\right)}{A_{i}} d F(i)$ is positive, $\frac{d P}{d \tau}$ is negative and viceversa. This proves lemma 1 . Note that we can write $\int_{i \in \mathcal{T}} \frac{\operatorname{sgn}\left(\Delta X_{1 i}\right)}{A_{i}} d F(i)$ as the difference between total buying and selling price derivatives at $\tau=0$, that is, $\int_{i \in \mathcal{B}} \frac{1}{A_{i}} d F(i)-\int_{i \in \mathcal{S}} \frac{1}{A_{i}} d F(i)=\mathbb{V a r}[D]\left(\frac{\partial\left(\left.\int_{i \in \mathcal{B}} X_{1 i}\right|_{\tau=0} d F(i)\right)}{\partial P}-\frac{\partial\left(\left.\int_{i \in \mathcal{S}} X_{1 i}\right|_{\tau=0} d F(i)\right)}{\partial P}\right)$, which shows the claim made in the text.

\section{Lemma 2. (Effect of $\tau$ on allocations)}

a) It must be shown that the sign of $\frac{d X_{1 i}}{d \tau}$ is identical to the one of $\frac{\partial X_{1 i}}{\partial \tau}$. The total derivative of portfolio allocations in equilibrium with respect to taxes is given by equation (8). Hence, as derived in the proof of lemma 1, $\frac{d P}{d \tau}=-\frac{P_{1} \int \frac{\operatorname{sgn}\left(\Delta X_{1 i}\right)}{A_{i}} d F(i)}{\int \frac{1+\operatorname{sgn}\left(\Delta X_{1 i}\right) \tau}{A_{i}} d F(i)}$. By substituting this expression in (8), along with $\frac{\partial X_{1 i}}{\partial \tau}=\frac{-P_{1} \operatorname{sgn}\left(\Delta X_{1 i}\right)}{A_{i} \mathbb{V} \operatorname{ar}[D]}$ and $\frac{\partial X_{1 i}}{\partial P}=\frac{-\left(1+\operatorname{sgn}\left(\Delta X_{1 i}\right) \tau\right)}{A_{i} \mathbb{V} a r[D]}$, we find:

$$
\frac{d X_{1 i}}{d \tau}=\frac{\partial X_{1 i}}{\partial \tau} \underbrace{\left[1-\left(\operatorname{sgn}\left(\Delta X_{1 i}\right)+\tau\right) \frac{\int \frac{\operatorname{sgn}\left(\Delta X_{1 i}\right)}{A_{i}} d F(i)}{\int \frac{1+\operatorname{sgn}\left(\Delta X_{1 i}\right) \tau}{A_{i}} d F(i)}\right]}_{\equiv \varepsilon_{i}}
$$

where $\varepsilon_{i}$, which is constant within buyers/sellers, can be rewritten as:

$$
\varepsilon_{i}=1-\left(\operatorname{sgn}\left(\Delta X_{1 i}\right)+\tau\right) \frac{1-H}{(1+\tau)+(1-\tau) H^{\prime}}
$$

where $H \equiv \frac{\int_{\mathcal{S}} \frac{1}{A_{i}} d F(i)}{\int_{\mathcal{B}} \frac{1}{A_{i}} d F(i)} \in(0, \infty)$ is the ratio of sellers risk aversion to buyers risk aversion. It is straightforward to show that $\varepsilon_{i}>0$ for both buyers and sellers. This proves lemma 2. 


\section{Proofs: Section 3}

\section{Assumption 3. [OBPS] (Optimists Buyers/Pessimists Sellers)}

The marginal gain/loss in welfare at $\tau=0$ is given by $-\left.\int \mathbb{E}_{i}[D] \frac{d X_{1 i}}{d \tau}\right|_{\tau=0} d F(i)$. We can define $\left.\int \mathbb{E}_{i}[D] \frac{d X_{1 i}}{d \tau}\right|_{\tau=0} d F(i) \equiv \mathbb{E}_{F}\left[\left.\mathbb{E}_{i}[D] \frac{d X_{1 i}}{d \tau}\right|_{\tau=0}\right]$, and then using properties of the covariance, find that $\mathbb{E}_{F}\left[\left.\mathbb{E}_{i}[D] \frac{d X_{1 i}}{d \tau}\right|_{\tau=0}\right]=\operatorname{Cov}_{F}\left(\mathbb{E}_{i}[D],\left.\frac{d X_{1 i}}{d \tau}\right|_{\tau=0}\right)+\mathbb{E}_{F}\left[\mathbb{E}_{i}[D]\right] \mathbb{E}_{F}\left[\left.\frac{d X_{1 i}}{d \tau}\right|_{\tau=0}\right]$. Market clearing implies that $\mathbb{E}_{F}\left[\frac{d X_{1 i}}{d \tau}\right]=0$, hence $\int \mathbb{E}_{i}[D] \frac{d X_{1 i}}{d \tau} d F(i)=\operatorname{Cov}_{F}\left(\mathbb{E}_{i}[D], \frac{d X_{1 i}}{d \tau}\right)$. This condition can be rewritten as a function of exogenous variables after substituting $\frac{d X_{1 i}}{d \tau}$.

\section{Proposition 1. (Marginal effect on welfare)}

a) By taking the derivative of social welfare, it follows that: $\frac{d V}{d \tau}=\int \lambda_{i} \frac{d V_{i}}{d \tau} d F(i)$. Taking the derivative of $V_{i}$ with respect to $\tau$, we can write:

$$
\frac{d V_{i}}{d \tau}=\mathbb{E}\left[U_{i}^{\prime}\left(W_{2 i}\right)\right]\left[\left[\mathbb{E}[D]-A_{i} \operatorname{Cov}\left[E_{2 i}, D\right]-P_{1}-A_{i} X_{1 i} \mathbb{V} \operatorname{ar}[D]\right] \frac{d X_{1 i}}{d \tau}-\Delta X_{1 i} \frac{d P_{1}}{d \tau}\right]
$$

The derivative $\frac{d X_{1 i}}{d \tau}$ is the equilibrium portfolio response. For those investors who decide not trade $\frac{d V_{i}}{d \tau}=0$, because $\frac{d X_{1 i}}{d \tau}=0$ and $X_{1 i}=X_{0 i}$. Note that to derive this equation, I am using an envelope condition in the extensive margin between trading and no trading. Substituting the first order condition (5) into the previous expression, we find:

$$
\frac{d V_{i}}{d \tau}=\mathbb{E}\left[U_{i}^{\prime}\left(W_{2 i}\right)\right]\left[\left[\mathbb{E}[D]-\mathbb{E}_{i}[D]+\operatorname{sgn}\left(\Delta X_{1 i}\right) P_{1} \tau\right] \frac{d X_{1 i}}{d \tau}-\Delta X_{1 i} \frac{d P_{1}}{d \tau}\right]
$$

Substituting this last expression into $\frac{d V}{d \tau}$, we recover the result in proposition 1a.

b) Imposing $\lambda_{i} \mathbb{E}_{i}\left[U_{i}^{\prime}\left(W_{2 i}\right)\right]=1$ allows us to write:

$$
\frac{d V_{i}}{d \tau}=\int\left[\left[\mathbb{E}[D]-\mathbb{E}_{i}[D]+\operatorname{sgn}\left(\Delta X_{1 i}\right) P_{1} \tau\right] \frac{d X_{1 i}}{d \tau}-\Delta X_{1 i} \frac{d P_{1}}{d \tau}\right] d F(i)
$$

Market clearing implies that $\int \Delta X_{1 i} d F(i)=0$, which allows to cancel the term multiplying $\frac{d P_{1}}{d \tau}$. Market clearing also implies that $\int \frac{d X_{1 i}}{d \tau} d F(i)=0$, which allows to cancel the term that corresponds to the planner's measure $\mathbb{E}[D]$. With this we recover equation (11).

c) Follows directly by imposing $\tau=0$ in (10).

\section{Corollary. (Irrelevance of planner's belief)}

Equation (10) can be rewritten as:

$$
\frac{d V}{d \tau}=\mathbb{E}[D] \int \lambda_{i} \mathbb{E}\left[U_{i}^{\prime}\left(W_{2 i}\right)\right] \frac{d X_{1 i}}{d \tau} d F(i)+\text { other terms }
$$

The expression for the optimal tax $\tau$ is not a function of $\mathbb{E}[D]$ if and only if $\int \lambda_{i} \mathbb{E}\left[U_{i}^{\prime}\left(W_{2 i}\right)\right] \frac{d X_{1 i}}{d \tau} d F(i)=$ 0 . When assumption [NR] holds, this condition becomes $\int \frac{d X_{1 i}}{d \tau} d F(i)=0$, which is always true due to market clearing. This shows the corollary. 


\section{Proposition 2. (Optimal financial transaction tax)}

a) The optimal financial transaction tax $\tau^{*}$ is given, in the general case, by:

$$
\tau^{*}=\frac{\frac{d P_{1}}{d \tau} \int \lambda_{i} \mathbb{E}\left[U_{i}^{\prime}\left(W_{2 i}\right)\right] \Delta X_{1 i} d F(i)}{P_{1} \int \lambda_{i} \mathbb{E}\left[U_{i}^{\prime}\left(W_{2 i}\right)\right] \operatorname{sgn}\left(\Delta X_{1 i}\right) \frac{d X_{1 i}}{d \tau} d F(i)}-\frac{\int \lambda_{i} \mathbb{E}\left[U_{i}^{\prime}\left(W_{2 i}\right)\right]\left(\mathbb{E}[D]-\mathbb{E}_{i}[D]\right) \frac{d X_{1 i}}{d \tau} d F(i)}{P_{1} \int \lambda_{i} \mathbb{E}\left[U_{i}^{\prime}\left(W_{2 i}\right)\right] \operatorname{sgn}\left(\Delta X_{1 i}\right) \frac{d X_{1 i}}{d \tau} d F(i)}
$$

The assumption equivalent to [OBPS] is determined by the term $\int \lambda_{i} \mathbb{E}\left[U_{i}^{\prime}\left(W_{2 i}\right)\right]\left(\mathbb{E}[D]-\mathbb{E}_{i}[D]\right) \frac{d X_{1 i}}{d \tau} d F(i)$. Imposing assumption $[\mathrm{NR}]$, and using market clearing as $\int \Delta X_{1 i} d F(i)=0$ and $\int \frac{d X_{1 i}}{d \tau} d F(i)=0$, we can write:

$$
\tau^{*}=\frac{\int \frac{\mathbb{E}_{i}[D]}{P_{1}} \frac{d X_{1 i}}{d \tau} d F(i)}{\int \operatorname{sgn}\left(\Delta X_{1 i}\right) \frac{d X_{1 i}}{d \tau} d F(i)}
$$

b) Because $-1<\tau<1$ and $V(\cdot)$ is continuous, by the extreme value theorem we conclude that there exists a maximum. I show in appendix $B$, section $B$, that when $\tau \leq 0$, the problem is strictly convex. This fact, combined with assumption [OBPS] guarantees that the maximum occurs in the $\tau>0$ region. Because of the non-convexity of the problem, equation 2 may have multiple solutions, which correspond to local optimum, but only one of them will be the global optimum.

Using market clearing, the denominator of the optimal tax can be written as:

$$
\int \operatorname{sgn}\left(\Delta X_{1 i}\right) \frac{d X_{1 i}}{d \tau} d F(i)=\int_{i \in \mathcal{B}} \frac{d X_{1 i}}{d \tau} d F(i)-\int_{i \in S} \frac{d X_{1 i}}{d \tau} d F(i)=2 \int_{i \in \mathcal{B}} \frac{d X_{1 i}}{d \tau} d F(i)
$$

Using lemma 2, this expression must be negative.

To recover equation (14), we can write:

$$
\tau^{*}=\frac{1}{2} \frac{\int \frac{\mathbb{E}_{i}[D]}{P_{1}} \frac{d X_{1 i}}{d \tau} d F(i)}{\int_{i \in \mathcal{B}} \frac{d X_{1 i}}{d \tau} d F(i)}=\frac{1}{2}[\overbrace{\int_{i \in \mathcal{B}} \frac{\mathbb{E}_{i}[D]}{P_{1}} \underbrace{\frac{\int_{i \in \mathcal{B}} \frac{d X_{1 i}}{d \tau} d F(i)}{\int_{i}}}_{\equiv \omega_{i}^{\mathcal{B}}} d F(i)}^{\equiv \Omega_{\mathcal{B}}}-\overbrace{\int_{i \in \mathcal{S}} \frac{\mathbb{E}_{i}[D]}{P_{1}} \underbrace{\frac{\frac{d X_{1 i}}{d \tau}}{\int_{i \in \mathcal{S}} \frac{d X_{1 i}}{d \tau} d F(i)}}_{\equiv \omega_{i}^{\mathcal{S}}} d F(i)}^{\equiv \Omega_{\mathcal{S}}}]
$$

Where I have used the fact that $\int \frac{\mathbb{E}_{i}[D]}{P_{1}} \frac{d X_{1 i}}{d \tau} d F(i)=\int_{i \in \mathcal{B}} \frac{\mathbb{E}_{i}[D]}{P_{1}} \frac{d X_{1 i}}{d \tau} d F(i)+\int_{i \in \mathcal{S}} \frac{\mathbb{E}_{i}[D]}{P_{1}} \frac{d X_{1 i}}{d \tau} d F(i)$ and that $\int_{i \in \mathcal{B}} \frac{d X_{1 i}}{d \tau} d F(i)=-\int_{i \in \mathcal{S}} \frac{d X_{1 i}}{d \tau} d F(i)$. Defining $\omega_{i} \equiv \frac{1}{2} \frac{d X_{1 i}}{d \tau} / \int_{i \in \mathcal{B}} \frac{d X_{1 i}}{d \tau} d F(i)$ and $\int \omega_{i} d F(i)=0$, we could also write the optimal tax as $\tau^{*}=\int \omega_{i} \frac{\mathbb{E}_{i}[D]}{P_{1}} d F(i)$.

\section{Proposition 3. (Harberger (1964) revisited)}

a) When investors assess probabilities according to the true probability distribution, we can write the marginal change in welfare as a money-metric (divided by investors' marginal utility) as:

$$
\left.\frac{d \hat{V}_{i}}{d \tau}\right|_{\tau=\tilde{\tau}} \equiv \frac{\frac{d V_{i}}{d \tau}}{\mathbb{E}\left[U_{i}^{\prime}\left(W_{2 i}\right)\right]}=\operatorname{sgn}\left(\Delta X_{1 i}\right) \tilde{\tau} P_{1} \frac{d X_{1 i}}{d \tau}-\Delta X_{1 i} \frac{d P_{1}}{d \tau}
$$

Adding up across all investors, and using the fact that $\int \operatorname{sgn}\left(\Delta X_{1 i}\right) \frac{d X_{1 i}}{d \tau} d F(i)=2 \int_{\mathcal{B}} \frac{d X_{1 i}}{d \tau} d F(i)$, we find:

$$
\int d \hat{V}_{i} d F(i)=2 \tilde{\tau} P_{1} \int_{i \in \mathcal{B}} \frac{d X_{1 i}}{d \tau} d \tau
$$

b) The result in a) is an exact expression. However, we can write a second order approximation around $\tau=0$ of the marginal change in social welfare. Note all terms corresponding to terms-oftrade cancel out after imposing market clearing, so I do not consider them. The first term of the Taylor 
expansion is given above. The derivative of the second term of the Taylor expansion is given by: $\operatorname{sgn}\left(\Delta X_{1 i}\right) P_{1} \frac{d X_{1 i}}{d \tau}+\operatorname{sgn}\left(\Delta X_{1 i}\right) \tau P_{1} \frac{d^{2} X_{1 i}}{d \tau^{2}}$. Around $\tau=0$, this becomes sgn $\left(\Delta X_{1 i}\right) P_{1} \frac{d X_{1 i}}{d \tau}$. Hence, when $\tau=0$ we can write:

$$
\begin{aligned}
\left.\int d \hat{V}_{i}\right|_{\tau=0} d F(i) \approx & \left.\int \operatorname{sgn}\left(\Delta X_{1 i}\right) \tau P_{1} \frac{d X_{1 i}}{d \tau}\right|_{\tau=0} d F(i)(d \tau) \\
& +\left.\frac{1}{2} \int\left(\operatorname{sgn}\left(\Delta X_{1 i}\right) P_{1} \frac{d X_{1 i}}{d \tau}+\operatorname{sgn}\left(\Delta X_{1 i}\right) \tau P_{1} \frac{d^{2} X_{1 i}}{d \tau^{2}}\right)\right|_{\tau=0} d F(i)(d \tau)^{2} \\
& =\left.\frac{1}{2} \int \operatorname{sgn}\left(\Delta X_{1 i}\right) P_{1} \frac{d X_{1 i}}{d \tau}\right|_{\tau=0} d F(i)(d \tau)^{2}=\left.P_{1} \tau^{2} \int_{i \in \mathcal{B}} \frac{d X_{1 i}}{d \tau}\right|_{\tau=0} d F(i)
\end{aligned}
$$

\section{Proofs: Section 5}

\section{Proposition 4. (Pre-existing trading costs)}

Given investors' optimal portfolios, stated in the main text, it is straightforward to derive the equilibrium price, which is given by:

$$
P_{1}=\frac{\int_{i \in \mathcal{T}} \frac{\mathbb{E}_{i}[D]-A_{i} \operatorname{Cov}\left[E_{2 i}, D\right]-\operatorname{sgn}\left(\Delta X_{1 i}\right) \eta+\alpha X_{0 i}}{A_{i} \mathbb{V a r}[D]+\alpha}-\int_{i \in \mathcal{T}} X_{0 i}}{\int_{i \in \mathcal{T}} \frac{\left(1+\operatorname{sgn}\left(\Delta X_{1 i}\right)(\tau+\psi)\right)}{A_{i} \mathbb{V a r}[D]+\alpha}}
$$

Indirect utility for an investor $i$ from the planner's perspective is given by:

$$
V_{i}=-e^{-A_{i}\left(\left(\mathbb{E}[D]-A_{i} \operatorname{Cov}\left[E_{2 i}, D\right]-P_{1}\right) X_{1 i}+P_{1} X_{0 i}-\left|\Delta X_{1 i}\right| P_{1} \psi-\frac{\alpha}{2}\left(\Delta X_{1 i}\right)^{2}-\frac{A_{i}}{2} \operatorname{Var}[D]\left(X_{1 i}\right)^{2}\right)}
$$

Note that only the resources corresponding to the transaction tax are rebated back to investors. All resources devoted to transaction costs are a compensation for the use of resources, so the planner does not have to account for them explicitly, since they form part of a zero profit condition.

Hence, the marginal change in welfare for an investor $i$ is given by:

$$
\frac{d V_{i}}{d \tau}=\mathbb{E}\left[U_{i}^{\prime}\left(W_{2 i}\right)\right]\left[\begin{array}{c}
\left(\mathbb{E}[D]-A_{i} \operatorname{Cov}\left[E_{2 i}, D\right]-P_{1}-\operatorname{sgn}\left(\Delta X_{1 i}\right) P_{1} \psi-\eta \operatorname{sgn}\left(\Delta X_{1 i}\right)-\alpha \Delta X_{1 i}-A \mathbb{V} a r[D] X_{1 i}\right) \frac{d X_{1 i}}{d \tau} \\
-\Delta X_{1 i} \frac{d P_{1}}{d \tau}
\end{array}\right]
$$

By substituting investors' first order conditions, we find:

$$
\frac{d V_{i}}{d \tau}=\mathbb{E}\left[U_{i}^{\prime}\left(W_{2 i}\right)\right]\left[\left[\mathbb{E}[D]-\mathbb{E}_{i}[D]+\operatorname{sgn}\left(\Delta X_{1 i}\right) P_{1} \tau\right] \frac{d X_{1 i}}{d \tau}-\Delta X_{1 i} \frac{d P_{1}}{d \tau}\right]
$$

Which is identical to the condition derived when showing proposition 1.

\section{Proposition 5. (Multiple risky assets)}

After getting rid of terms that do not affect the maximization problem, investors solve:

$$
\max _{\mathbf{x}_{1 i}} \mathbf{x}_{1 i}^{\prime}\left(\mathbb{E}_{i}[\mathbf{d}]-A_{i} \operatorname{Cov}\left[E_{2 i}, \mathbf{d}\right]-\mathbf{p}\right)-\left|\mathbf{x}_{1 i}^{\prime}-\mathbf{x}_{0 i}^{\prime}\right| \mathbf{p} \tau-\frac{A_{i}}{2} \mathbf{x}_{1 i}^{\prime} \mathbb{V} \operatorname{ar}[\mathbf{d}] \mathbf{x}_{1 i}
$$

Where I use $\left|\mathbf{x}_{1 i}^{\prime}-\mathbf{x}_{0 i}^{\prime}\right|$ to denote the vector of absolute values of the difference between both vectors. This problem is convex, so the first order condition fully characterizes investors' optimal portfolios as long as they trade a given asset $j$ :

$$
\mathbf{x}_{1 i}=\left(A_{i} \mathbb{V} \operatorname{ar}[\mathbf{d}]\right)^{-1}\left(\mathbb{E}_{i}[\mathbf{d}]-A_{i} \operatorname{Cov}\left[E_{2 i}, \mathbf{d}\right]-\mathbf{p}-\hat{\mathbf{p}}_{i} \tau\right),
$$


where $\hat{\mathbf{p}}_{i}$ is a $J \times 1$ vector where a given row $j$ is given by $\operatorname{sgn}\left(\Delta X_{1 i j}\right) p_{j}$. If an asset $j$ is not traded by an investor $i$, then $X_{1 i j}=X_{0 i j}$. The inaction regions are defined analogously to the one asset case. Note that there exists a way to write optimal portfolio choices only with matrix operations; however, the notation turns out to be more cumbersome. The equilibrium price vector is given by:

$$
\mathbf{p} \hat{\times} \int\left(1+\frac{\mathbf{s}_{i}}{A_{i}} \tau\right) d F(i)=\int \frac{\mathbb{E}_{i}[\mathbf{d}]}{A_{i}} d F(i)-\int\left(\operatorname{Cov}\left[E_{2 i}, \mathbf{d}\right]+\operatorname{Var}[\mathbf{d}] \mathbf{x}_{0 i}\right) d F(i)
$$

Where I denote element-by-element multiplication as $y \hat{\times} z$ and use $\mathbf{s}_{i}$ to denote a $J \times 1$ vector given by $\operatorname{sgn}\left(\Delta X_{1 i j}\right)$.

The marginal effect of varying taxes in social welfare is given by:

$$
\frac{d V}{d \tau}=\int \lambda_{i} \mathbb{E}\left[U_{i}^{\prime}\left(W_{2 i}\right)\right]\left[\left(\mathbb{E}[\mathbf{d}]-\mathbb{E}_{i}[\mathbf{d}]+\hat{\mathbf{p}}_{i} \tau\right)^{\prime} \frac{d \mathbf{x}_{1 i}}{d \tau}-\left(\mathbf{x}_{1 i}-\mathbf{x}_{0 i}\right)^{\prime} \frac{d \mathbf{p}}{d \tau}\right] d F(i)
$$

This is a generalization of the one asset case. Under assumption 2, we can write in product notation:

$$
\int \sum_{j=1}^{J}\left(-\mathbb{E}_{i}\left[D_{j}\right]+\operatorname{sgn}\left(\Delta X_{1 i j}\right) p_{j} \tau\right) \frac{d X_{1 i j}}{d \tau} d F(i)=0
$$

So the optimal tax becomes:

$$
\tau^{*}=\frac{\sum_{j=1}^{J} \int \mathbb{E}_{i}\left[D_{j}\right] \frac{d X_{1 i j}}{d \tau} d F(i)}{\sum_{j=1}^{J} \int \operatorname{sgn}\left(\Delta X_{1 i j}\right) p_{j} \frac{d X_{1 i j}}{d \tau} d F(i)}
$$

Which can be rewritten as:

$$
\tau^{*}=\frac{\sum_{j=1}^{J} \int \operatorname{sgn}\left(\Delta X_{1 i j}\right) p_{j} \frac{d X_{1 i j}}{d \tau} d F(i) \tau_{j}^{*}}{\sum_{j=1}^{J} \int \operatorname{sgn}\left(\Delta X_{1 i j}\right) p_{j} \frac{d X_{1 i j}}{d \tau} d F(i)}
$$

Where $\tau_{j}^{*}=\frac{\int \frac{\mathbb{E}_{i}\left[D_{j}\right]}{p_{j}} \frac{d X_{1 i j}}{d \tau} d F(i)}{\int \operatorname{sgn}\left(\Delta X_{1 i j}\right) \frac{d X_{1 i j}}{d \tau} d F(i)}$. And by defining weights $\omega_{j}=\frac{\int \operatorname{sgn}\left(\Delta X_{1 i j}\right) p_{j} \frac{d X_{1 i j}}{d \tau} d F(i)}{\sum_{j=1}^{J} \int \operatorname{sgn}\left(\Delta X_{1 i j}\right) p_{j} \frac{d X_{1 i j}}{d \tau} d F(i)}$, we recover equation (19).

\section{Proposition 6. (Trading constraints)}

The equilibrium price is given by:

$$
P_{1}=\frac{\int_{i \in \mathcal{T}, U} \frac{\mathbb{E}_{i}[D]-A_{i} \operatorname{Cov}\left[E_{2 i}, D\right]}{A_{i} \mathbb{V a r}[D]}-\int_{i \in \mathcal{T}} X_{0 i}-\int_{i \in \mathcal{C}} g_{i}\left(P_{1}\right)}{\int_{i \in \mathcal{T}, U} \frac{1+\operatorname{sgn}\left(\Delta X_{1 i}\right) \tau}{A_{i} \mathbb{V a r}[D]}},
$$

where $i \in \mathcal{T}, U$ denotes the set of active unconstrained investors.

The change in social welfare for an investor $i$, from a planner's perspective, is given by:

$$
\frac{d V_{i}}{d \tau}=\mathbb{E}\left[U_{i}^{\prime}\left(W_{2 i}\right)\right]\left[\left[\mathbb{E}[D]-A_{i} \operatorname{Cov}\left[E_{2 i}, D\right]-P_{1}-A_{i} X_{1 i} \mathbb{V} \operatorname{ar}[D]\right] \frac{d X_{1 i}}{d \tau}-\Delta X_{1 i} \frac{d P_{1}}{d \tau}\right]
$$

I use $i=U$ to denote unconstrained investors and $i=C$ for constrained investors. Substituting the optimality condition:

$$
\begin{gathered}
\left.\frac{d V_{i}}{d \tau}\right|_{i=U}=\mathbb{E}\left[U_{i}^{\prime}\left(W_{2 i}\right)\right]\left[\left[\mathbb{E}[D]-\mathbb{E}_{i}[D]+\operatorname{sgn}\left(\Delta X_{1 i}\right) P_{1} \tau\right] \frac{d X_{1 i}}{d \tau}-\Delta X_{1 i} \frac{d P_{1}}{d \tau}\right] \\
\left.\frac{d V_{i}}{d \tau}\right|_{i=C}=\mathbb{E}\left[U_{i}^{\prime}\left(W_{2 i}\right)\right]\left[\left[\mathbb{E}[D]-A_{i} \operatorname{Cov}\left[E_{2 i}, D\right]-P_{1}-A_{i} g\left(P_{1}\right) \mathbb{V a r}[D]\right] \frac{d X_{1 i}}{d \tau}-\Delta X_{1 i} \frac{d P_{1}}{d \tau}\right]
\end{gathered}
$$


We can show that the term multiplying $\frac{d X_{1 i}}{d \tau}$ for constrained investors is positive when:

$$
g\left(P_{1}\right)<\frac{\mathbb{E}[D]-A_{i} \operatorname{Cov}\left[E_{2 i}, D\right]-P_{1}}{A_{i} \mathbb{V a r}[D]}
$$

and negative otherwise. The welfare change for constrained investors can be rewritten, by substituting the (shadow) first order condition as:

$$
\left.\frac{d V_{i}}{d \tau}\right|_{i=C}=\mathbb{E}\left[U_{i}^{\prime}\left(W_{2 i}\right)\right]\left[\begin{array}{c}
{\left[\mathbb{E}[D]-\mathbb{E}_{i}[D]+A_{i} \mathbb{V a r}[D]\left(\hat{X}_{1 i}-g\left(P_{1}\right)\right)+P_{1} \operatorname{sgn}\left(\Delta X_{1 i}\right) \tau\right] \frac{d X_{1 i}}{d \tau}} \\
-\Delta X_{1 i} \frac{d P_{1}}{d \tau}
\end{array}\right]
$$

Under assumption 2, we can write social welfare as:

$$
\frac{d V}{d \tau}=\int\left[-\mathbb{E}_{i}[D]+\operatorname{sgn}\left(\Delta X_{1 i}\right) P_{1} \tau\right] \frac{d X_{1 i}}{d \tau} d F(i)+\int_{i \in \mathcal{C}} A_{i} \mathbb{V} \operatorname{ar}[D]\left(\hat{X}_{1 i}-g\left(P_{1}\right)\right) \frac{d X_{1 i}}{d \tau} d F(i)
$$

Where I use $\mathcal{C}$ to denote the set of constrained investors and $\hat{X}_{1 i}$ is given by the individual first order condition in equation (5). After substituting for constrained investors $\frac{d X_{1 i}}{d \tau}=g^{\prime}\left(P_{1}\right) \frac{d P_{1}}{d \tau}$, we can write the optimal tax as:

$$
\begin{gathered}
\int\left[-\mathbb{E}_{i}[D]+\operatorname{sgn}\left(\Delta X_{1 i}\right) P_{1} \tau\right] \frac{d X_{1 i}}{d \tau} d F(i)+\int_{i \in \mathcal{C}} A_{i} \mathbb{V} \operatorname{ar}[D]\left(\hat{X}_{1 i}-g\left(P_{1}\right)\right) g^{\prime}\left(P_{1}\right) \frac{d P_{1}}{d \tau} d F(i)=0 \\
\tau^{*}=\frac{\int \mathbb{E}_{i}[D] \frac{d X_{1 i}}{d \tau} d F(i)-\int_{i \in \mathcal{C}} A_{i} \mathbb{V} \operatorname{ar}[D]\left(\hat{X}_{1 i}-g\left(P_{1}\right)\right) g^{\prime}\left(P_{1}\right) \frac{d P_{1}}{d \tau} d F(i)}{P_{1} \int \operatorname{sgn}\left(\Delta X_{1 i}\right) \frac{d X_{1 i}}{d \tau} d F(i)}
\end{gathered}
$$

\section{Proposition 7. (Disagreement about second moments)}

The optimal portfolio allocation for an investor $i$ in his trade region is given by:

$$
X_{1 i}=\frac{\mathbb{E}_{i}[D]-A_{i} \operatorname{Cov}_{i}\left[E_{2 i}, D\right]-P_{1}\left(1+\operatorname{sgn}\left(\Delta X_{1 i}\right) \tau\right)}{A_{i} \mathbb{V} a r_{i}[D]}
$$

The marginal change in welfare for an investor $i$ is given by:

$$
\frac{d V_{i}}{d \tau}=\mathbb{E}\left[U_{i}^{\prime}\left(W_{2 i}\right)\right]\left[\left(\mathbb{E}[D]-A_{i} \operatorname{Cov}\left[E_{2 i}, D\right]-P_{1}-A_{i} X_{1 i} \mathbb{V} a r[D]\right) \frac{d X_{1 i}}{d \tau}-\Delta X_{1 i} \frac{d P_{1}}{d \tau}\right]
$$

Substituting the individual optimality condition, we find:

$$
\frac{\frac{d V_{i}}{d \tau}}{\mathbb{E}\left[U_{i}^{\prime}\left(W_{2 i}\right)\right]}=\left[\begin{array}{c}
\left(\mathbb{E}[D]-A_{i} \operatorname{Cov}\left[E_{2 i}, D\right]-P_{1}-r_{i}\left(\mathbb{E}_{i}[D]-A_{i} \operatorname{Cov}_{i}\left[E_{2 i}, D\right]-P_{1}\left(1+\operatorname{sgn}\left(\Delta X_{1 i}\right) \tau\right)\right)\right) \frac{d X_{1 i}}{d \tau} \\
-\Delta X_{1 i} \frac{d P_{1}}{d \tau}
\end{array}\right]
$$

Where $r_{i} \equiv \frac{\mathbb{V} a r[D]}{\mathbb{V} a r_{i}[D]}$. The change in social welfare can then be written, under assumption 2, as:

$$
\frac{d V}{d \tau}=\int\left(-r_{i} \mathbb{E}_{i}[D]-A_{i} \operatorname{Cov}\left[E_{2 i}, D\right]\left(1-\frac{\beta_{i i}}{\beta_{i}}\right)+P_{1} r_{i}\left(1+\operatorname{sgn}\left(\Delta X_{1 i}\right) \tau\right)\right) \frac{d X_{1 i}}{d \tau} d F(i)
$$

Solving for $\tau$ in this equation, which corresponds to equation 21 in the paper, delivers the expression for the optimal tax in proposition $7 \mathrm{~b}$. 


\section{Proposition 8. (Asymmetric taxes on buyers versus sellers)}

The budget constraint for an investor in this case can be written as:

$$
W_{2 i}=E_{2 i}+X_{1 i} D+\left(X_{0 i} P_{1}-X_{1 i} P_{1}-\tau_{B}\left|P_{1}\right|\left|\Delta X_{1 i}\right|_{+}-\tau_{S}\left|P_{1}\right|\left|\Delta X_{1 i}\right|_{-}+T_{1 i}\right)
$$

The first order condition becomes:

$$
X_{1 i}=\frac{\mathbb{E}_{i}[D]-A_{i} \operatorname{Cov}\left[E_{2 i}, D\right]-P_{1}\left(1+\mathbb{I}\left[\Delta X_{1 i}>0\right] \tau_{B}+\mathbb{I}\left[\Delta X_{1 i}<0\right] \tau_{S}\right)}{A_{i} \mathbb{V} \operatorname{ar}[D]}
$$

With an equilibrium price:

$$
P_{1}=\frac{\int_{i \in \mathcal{T}}\left(\frac{\mathbb{E}_{i}[D]-A_{i} \operatorname{Cov}\left[E_{2 i}, D\right]}{A_{i}}-\operatorname{Var}[D] X_{0 i}\right) d F(i)}{\int_{i \in \mathcal{T}} \frac{1}{A_{i}}+\tau_{B} \int_{i \in \mathcal{B}} \frac{1}{A_{i}}-\tau_{S} \int_{i \in \mathcal{S}} \frac{1}{A_{i}} d F(i)}
$$

In this case we can write: $X_{1 i}\left(\tau_{i}, P_{1}\left(\left\{\tau_{j}\right\}\right)\right)$, where $\left\{\tau_{j}\right\}$ denotes a vector of taxes. This implies that $\frac{d X_{1 i}}{d \tau_{j}}=\frac{\partial X_{1 i}}{\partial \tau_{j}}+\frac{\partial X_{1 i}}{\partial P_{1}} \frac{d P_{1}}{d \tau_{j}}$. The change in social welfare for an investor $i$ when varying a tax $\tau_{j}$, from a planner's perspective, is given by:

$$
\begin{aligned}
\frac{d V_{i}}{d \tau_{j}} & =\mathbb{E}\left[U_{i}^{\prime}\left(W_{2 i}\right)\right]\left[\left(\mathbb{E}[D]-A_{i} \operatorname{Cov}\left[E_{2 i}, D\right]-P_{1}-A_{i} X_{1 i} \mathbb{V} \text { ar }[D]\right) \frac{d X_{1 i}}{d \tau_{j}}-\Delta X_{1 i} \frac{d P_{1}}{d \tau_{j}}\right] \\
& =\mathbb{E}\left[U_{i}^{\prime}\left(W_{2 i}\right)\right]\left(\left(\mathbb{E}[D]-\mathbb{E}_{i}[D]+P_{1}\left(\mathbb{I}\left[\Delta X_{1 i}>0\right] \tau_{B}-\mathbb{I}\left[\Delta X_{1 i}<0\right] \tau_{S}\right)\right) \frac{d X_{1 i}}{d \tau_{j}}-\Delta X_{1 i} \frac{d P_{1}}{d \tau_{j}}\right)
\end{aligned}
$$

Social welfare is then:

$$
\frac{d V}{d \tau_{j}}=\int \lambda_{i} \mathbb{E}\left[U_{i}^{\prime}\left(W_{2 i}\right)\right]\left(\left(\mathbb{E}[D]-\mathbb{E}_{i}[D]+P_{1}\left(\mathbb{I}\left[\Delta X_{1 i}>0\right] \tau_{B}-\mathbb{I}\left[\Delta X_{1 i}<0\right] \tau_{S}\right)\right) \frac{d X_{1 i}}{d \tau_{j}}-\Delta X_{1 i} \frac{d P_{1}}{d \tau_{j}}\right) d F(i)
$$

Any tax change has two direct effects. First, it marginally affects those agents who pay that tax at the margin. Second, it moves prices. This price change creates two effects. There is a first effect working through terms-of-trade. A second effect works through demand changes. Under the usual differentiability and convexity assumptions, the optimal tax is characterized by $\frac{d V}{d \tau_{j}}=0, \forall j$. This yields a system of equation in the vector of taxes:

$$
0=\int \lambda_{i} \mathbb{E}\left[U_{i}^{\prime}\left(W_{2 i}\right)\right]\left(\left(\mathbb{E}[D]-\mathbb{E}_{i}[D]+P_{1} \operatorname{sgn}\left(\Delta X_{1 i}\right) \tau_{i}\right) \frac{d X_{1 i}}{d \tau_{j}}-\Delta X_{1 i} \frac{d P_{1}}{d \tau_{j}}\right) d F(i), \forall j
$$

This equation characterizes a system of equations in $\tau_{B}$ and $\tau_{S}$.

When [NR] holds, that is $\lambda_{i} \mathbb{E}\left[U_{i}^{\prime}\left(W_{2 i}\right)\right]=1, \forall i$, we can write:

$$
\frac{d V}{d \tau_{j}}=\int\left(\mathbb{E}[D]-\mathbb{E}_{i}[D]\right) \frac{d X_{1 i}}{d \tau_{j}} d F(i)+P_{1}\left(\tau_{B} \int_{i \in \mathcal{B}} \frac{d X_{1 i}}{d \tau_{j}} d F(i)-\tau_{S} \int_{i \in \mathcal{S}} \frac{d X_{1 i}}{d \tau_{j}} d F(i)\right)
$$

Using market clearing, we can find:

$$
\frac{d V}{d \tau_{j}}=-\int \mathbb{E}_{i}[D] \frac{d X_{1 i}}{d \tau_{j}} d F(i)+P_{1}\left(\tau_{B}+\tau_{S}\right) \int_{i \in \mathcal{B}} \frac{d X_{1 i}}{d \tau_{j}} d F(i)
$$

Solving for $\tau_{B}+\tau_{S}$ :

$$
\tau_{B}+\tau_{S}=\frac{\int \mathbb{E}_{i}[D] \frac{d X_{1 i}}{d \tau_{j}} d F(i)}{P_{1} \int_{i \in \mathcal{B}} \frac{d X_{1 i}}{d \tau_{j}} d F(i)}, \forall_{j}
$$


In general this gives a system of non-linear equations in $\tau_{B}+\tau_{S}$. When there are two agents, the two equations become collinear, because of market clearing:

$$
\int \mathbb{E}_{i}[D] \frac{d X_{1 i}}{d \tau_{j}} d F(i)=\left(\mathbb{E}_{\mathcal{B}}[D]-\mathbb{E}_{S}[D]\right) \int_{i \in \mathcal{B}} \frac{d X_{1 i}}{d \tau_{j}} d F(i)
$$

In that case only the sum of taxes is pinned down:

$$
\tau_{B}+\tau_{S}=\frac{\mathbb{E}_{\mathcal{B}}[D]-\mathbb{E}_{\mathcal{S}}[D]}{P_{1}}
$$

\section{Proposition 9. (Individual taxes/First-best)}

a) In the case with $I$ taxes and $I$ agents, the first order conditions under assumption [NR] for the planner become:

$$
\frac{d V}{d \tau_{j}}=\sum_{i}\left(-\mathbb{E}_{i}[D]+P_{1} \operatorname{sgn}\left(\Delta X_{1 i}\right) \tau_{i}\right) \frac{d X_{1 i}}{d \tau_{j}} F(i)=0, \forall j
$$

This system of equations characterizes the set of optimal taxes. Note that one solution to this system is given by:

$$
-\mathbb{E}_{i}[D]+P_{1} \operatorname{sgn}\left(\Delta X_{1 i}\right) \tau_{i}=-F
$$

Where $F$ is an arbitrary real number. Rearranging this expression we can find equation (23).

b) Starting from the system of equations which characterizes the optimal set of taxes, we can write, using market clearing $F(j) \frac{d X_{1 i}}{d \tau_{j}}+\sum_{i \neq j} \frac{d X_{1 i}}{d \tau_{j}} F(i)=0$, the following set of equations:

$\sum_{i \neq j}\left(\mathbb{E}_{j}[D]-\mathbb{E}_{i}[D]\right) \frac{d X_{1 i}}{d \tau_{j}} F(i)+P_{1}\left(-\operatorname{sgn}\left(X_{1 j}-X_{0 j}\right) \tau_{j} \sum_{i \neq j} \frac{d X_{1 i}}{d \tau_{j}} F(i)+\sum_{i}\left(\operatorname{sgn}\left(\Delta X_{1 i}\right) \tau_{i} \frac{d X_{1 i}}{d \tau_{j}}\right) F(i)\right)=0$

For all equations but for the one with respect to tax $j$. To show that this system only depends on $N-1$ taxes, we simply need to show that all $\frac{d X_{1 i}}{d \tau_{j}}$ do not depend on the tax $\tau_{j}$. Note that $\frac{d X_{1 i}}{d \tau_{j}}=\frac{\partial X_{1 i}}{\partial \tau_{j}}+\frac{\partial X_{1 i}}{\partial P_{1}} \frac{d P_{1}}{d \tau_{j}}$. But when $i \neq j$ then $\frac{d X_{1 i}}{d \tau_{j}}$ only depends on $\frac{d P_{1}}{d \tau_{j}}$ because $\frac{\partial X_{1 i}}{\partial \tau_{j}}$ equals zero and $\frac{\partial X_{1 i}}{\partial P_{1}}$ does not depend on $\tau_{j}$. We just need to show that $\frac{d P_{1}}{d \tau_{j}}$ can be written as a function of all other taxes but $\tau_{j}$. This can be easily shown combining the expressions used to show lemma 1 with market clearing conditions.

\section{Proofs: Section 6}

\section{Equilibrium definition}

A competitive equilibrium with taxes is defined as a portfolio allocation $X_{t i}, Y_{t i}$ and consumption allocation $C_{t i}$, for every investor, a price $P_{t}$ and a set of lump-sum transfers $T_{t i}$ for every period and state such that: investors maximize (24) subject to their period by period constraint (25); the price $P_{t}$ is such the market for the risky asset clears every period, that is $\int X_{t i} d F(i)=Q$ and tax revenues are rebated in lump-sum fashion to investors in the following way: $T_{t i}=\tau P_{1}\left|\Delta X_{t i}\right|$.

\section{Proposition 10. (Marginal effect on welfare/Optimal dynamic tax)}

a) The individual problem can be written as:

$$
\max _{C_{t i}, X_{t i}, Y_{t i}} \mathbb{E}_{i}\left[\sum_{t=1}^{T} \beta^{t-1} U_{i}\left(C_{t i}\right)\right]
$$


Subject to:

$$
C_{t i}=E_{t i}+X_{t-1 i}\left(P_{t}+D_{t}\right)-\left|\Delta X_{t i}\right| P_{t} \tau+T_{t i}-X_{t i} P_{t}+R Y_{t-1}-Y_{t i} \text { for } t=1, \ldots, T-1
$$

And $C_{T i}=E_{T i}+X_{t-1 i} D_{T}+R Y_{T-1}$. We can iterate the budget constraint and find:

$$
C_{t+1 i}=E_{t+1 i}+X_{t i}\left(P_{t+1}+D_{t+1}\right)-\left|\Delta X_{t+1 i}\right| P_{t+1} \tau+T_{t+1 i}-X_{t+1 i} P_{1 t+1}+R Y_{t}-Y_{t+1 i}
$$

The Euler equations for each investor in addition to market clearing conditions characterize fully characterize the equilibrium. Standard variational arguments allow us to derive equations (26) and (27) in the paper. An alternative recursive representation would yield the same conditions — the derivations following this approach are available under request.

Indirect utility for an investor $i$ from the planner's perspective is given by:

$$
V_{i}=\mathbb{E}\left[\sum_{t=1}^{T} \beta^{t-1} U_{i}\left(E_{t i}+X_{t-1 i}\left(P_{t}+D_{t}\right)-X_{t i} P_{t}+R Y_{t-1}-Y_{t i}\right)\right]
$$

A marginal change in $\tau$ changes $V_{i}$ in the following way:

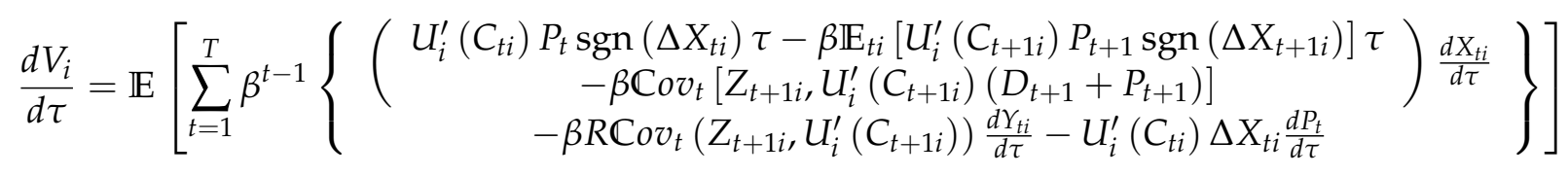

This expression corresponds to equation (30) in the paper. The marginal change in social welfare from varying the financial transaction tax is thus given by:

$$
\frac{d V}{d \tau}=\mathbb{E}\left[\sum_{t=1}^{T} \int \beta^{t-1} \lambda_{i}\left\{\begin{array}{c}
\left(\begin{array}{c}
U_{i}^{\prime}\left(C_{t i}\right) P_{t} \operatorname{sgn}\left(\Delta X_{t i}\right) \tau \\
-\beta \mathbb{E}_{t i}\left[U_{i}^{\prime}\left(C_{t+1 i}\right) P_{t+1} \operatorname{sgn}\left(\Delta X_{t+1 i}\right)\right] \tau \\
-\beta \operatorname{Cov}_{t}\left[Z_{t+1 i}, U_{i}^{\prime}\left(C_{t+1 i}\right)\left(D_{t+1}+P_{t+1}\right)\right] \\
-\beta R \operatorname{Cov}_{t}\left(Z_{t+1 i}, U_{i}^{\prime}\left(C_{t+1 i}\right) \frac{d Y_{t i}}{d \tau}-U_{i}^{\prime}\left(C_{t i}\right) \Delta X_{t i} \frac{d P_{t}}{d \tau}\right.
\end{array}\right\} d F(i) \\
-\beta C_{i j}
\end{array}\right]\right.
$$

Approximating this equation around $U_{i}^{\prime}\left(C_{t i}\right) \approx 1$, we find, after using market clearing:

$$
\frac{d V}{d \tau}=\mathbb{E}\left[\sum_{t=1}^{T} \int \beta^{t-1} \lambda_{i}\left(\begin{array}{c}
P_{t} \operatorname{sgn}\left(\Delta X_{t i}\right) \tau-\mathbb{E}_{t i}\left[P_{t+1} \operatorname{sgn}\left(\Delta X_{t+1 i}\right)\right] \tau \\
-\operatorname{Cov}_{t}\left[Z_{t+1 i}\left(D_{t+1}+P_{t+1}\right)\right]
\end{array}\right) \frac{d X_{t i}}{d \tau} d F(i)\right]
$$

Equation (31) corresponds to the $\beta=1$ case.

b) The general optimal financial transaction $\operatorname{tax} \tau^{*}$ is given by:

$\tau^{*}=\frac{-\mathbb{E}\left[\sum_{t=1}^{T} \int \beta^{t-1} \lambda_{i}\left[\beta \operatorname{Cov}_{t}\left[Z_{t+1 i}, U_{i}^{\prime}\left(C_{t+1 i}\right)\left(D_{t+1}+P_{t+1}\right)\right] \frac{d X_{t i}}{d \tau}+\beta R \operatorname{Cov}_{t}\left(Z_{t+1 i}, U_{i}^{\prime}\left(C_{t+1 i}\right)\right) \frac{d Y_{t i}}{d \tau}+g_{t}\right] d F(i)\right]}{\mathbb{E}\left[\sum_{t=1}^{T} \int \beta^{t-1} \lambda_{i}\left[U_{i}^{\prime}\left(C_{t i}\right) P_{t} \operatorname{sgn}\left(\Delta X_{t i}\right)-\beta \mathbb{E}_{t i}\left[U_{i}^{\prime}\left(C_{t+1 i}\right) P_{t+1} \operatorname{sgn}\left(\Delta X_{t+1 i}\right)\right]\right] \frac{d X_{t i}}{d \tau} d F(i)\right]}$,

where $g_{t} \equiv U_{i}^{\prime}\left(C_{t i}\right) \Delta X_{t i} \frac{d P_{t}}{d \tau}$. The numerator has three terms: the wedge in the portfolio allocation of the risky asset., the wedge caused by belief distortions in precautionary savings and the effect of the tax on terms-of-trade.

Using assumption [CMU/NR], that is, imposing $\lambda_{i}=1$ and substituting $U_{i}^{\prime}\left(C_{t i}\right)=1$, substituting the Euler equation for the riskless asset and using the properties of covariances we can write:

$$
\tau^{*}=\frac{-\mathbb{E}\left[\sum_{t=1}^{T} \beta^{t} \int \operatorname{Cov}_{t}\left[Z_{t+1 i}\left(D_{t+1}+P_{t+1}\right)\right] \frac{d X_{t i}}{d \tau} d F(i)\right]}{\mathbb{E}\left[\sum_{t=1}^{T} \beta^{t} \int\left[P_{t} \operatorname{sgn}\left(\Delta X_{t i}\right)-\mathbb{E}_{t i}\left[P_{t+1} \operatorname{sgn}\left(\Delta X_{t+1 i}\right)\right]\right] \frac{d X_{t i}}{d \tau} d F(i)\right]},
$$


where I have used market clearing, that is $\int \Delta X_{t i} d F(i)=0, \forall t$. Note that:

$$
\operatorname{Cov}_{t}\left[Z_{t+1 i},\left(D_{t+1}+P_{t+1}\right)\right]=\mathbb{E}_{t i}\left[D_{t+1}+P_{t+1}\right]-\mathbb{E}_{t}\left[D_{t+1}+P_{t+1}\right]
$$

Hence:

$$
\tau^{*}=\frac{\mathbb{E}\left[\sum_{t=1}^{T} \beta^{t} \int\left(\mathbb{E}_{t}\left[D_{t+1}+P_{t+1}\right]-\mathbb{E}_{t i}\left[D_{t+1}+P_{t+1}\right]\right) \frac{d X_{t i}}{d \tau} d F(i)\right]}{\mathbb{E}\left[\sum_{t=1}^{T} \beta^{t} \int\left[P_{t} \operatorname{sgn}\left(\Delta X_{t i}\right)-\mathbb{E}_{t i}\left[P_{t+1} \operatorname{sgn}\left(\Delta X_{t+1 i}\right)\right]\right] \frac{d X_{t i}}{d \tau} d F(i)\right]}
$$

And using market clearing, that is $\int \frac{d X_{t i}}{d \tau}=0$, and rearranging the denominator, we can write:

$$
\tau^{*}=\frac{\mathbb{E}\left[\sum_{t=1}^{T} \beta^{t} \int \mathbb{E}_{t i}\left[D_{t+1}+P_{t+1}\right] \frac{d X_{t i}}{d \tau} d F(i)\right]}{\mathbb{E}\left[\sum_{t=1}^{T} \beta^{t} \int\left[P_{t} \operatorname{sgn}\left(\Delta X_{t i}\right)\left(1-\kappa_{t i}\right)\right] \frac{d X_{t i}}{d \tau} d F(i)\right]},
$$

where $\kappa_{t i} \equiv \mathbb{E}_{t i}\left[\frac{P_{t+1}}{P_{t}} \operatorname{sgn}\left(\Delta X_{t i}\right) \operatorname{sgn}\left(\Delta X_{t+1 i}\right)\right]$. This expression corresponds to equation (32) in the text when $\beta=1$.

\section{Discussion $\frac{\mathbb{E}_{t i}\left[P_{t+1}\right]}{P_{t}} \neq 1$}

When $\frac{\mathbb{E}_{t i}\left[P_{t+1}\right]}{P_{t}} \neq 1$ an investor who sells shares at period $t$ when $\tau=0$ could become a buyer at period $t$ if $\tau$ is sufficiently large. More surprisingly, it may be the case that the portfolio derivative for a buyer at period becomes positive - this is the opposite of the result shown in lemma 1. All the formulas derived in the paper can handle that case directly. However, for interpretation, note that when $1-\kappa_{t i}$ is negative, we still expect sgn $\left(\Delta X_{t i}\right)\left(1-\kappa_{t i}\right) \frac{d X_{t i}}{d \tau}$ to be positive - guaranteeing that the denominator in (32) is strictly positive. In that case, buyers (sellers) would have $\frac{d X_{t i}}{d \tau}>0(<0)$, so this would not change the sign denominator of the optimal tax. Intuitively, a tax increase makes the tax more onerous at $t+1$ than at $t$, so it is optimal for a permanent buyer to acquire more shares of the risky asset at $t$.

\section{Corollary. (Optimal dynamic tax formula)}

a) Starting from the optimal tax formula, by defining:

$$
\tau_{t}^{*} \equiv \frac{\int \mathbb{E}_{t i}\left[D_{t+1}+P_{t+1}\right] \frac{d X_{t i}}{d \tau} d F(i)}{\int P_{t} \operatorname{sgn}\left(\Delta X_{t i}\right) \frac{d X_{t i}}{d \tau} d F(i)},
$$

we can write:

$$
\tau^{*}=\frac{\mathbb{E}\left[\sum_{t=1}^{T} \beta^{t}\left(\int P_{t} \operatorname{sgn}\left(\Delta X_{t i}\right) \frac{d X_{t i}}{d \tau} d F(i)\right) \tau_{t}^{*}\right]}{\mathbb{E}\left[\sum_{t=1}^{T} \beta^{t} \int\left[P_{t} \operatorname{sgn}\left(\Delta X_{t i}\right)\left(1-\kappa_{t i}\right)\right] \frac{d X_{t i}}{d \tau} d F(i)\right]}
$$

Hence, by defining:

$$
f_{t} \equiv \frac{\int \operatorname{sgn}\left(\Delta X_{t i}\right) \frac{d X_{t i}}{d \tau} d F(i)}{\int \operatorname{sgn}\left(\Delta X_{t i}\right)\left(1-\kappa_{t i}\right) \frac{d X_{t i}}{d \tau} d F(i)}
$$

we can write:

$$
\tau^{*}=\frac{\mathbb{E}\left[\sum_{t=1}^{T} \beta^{t}\left(\int P_{t} \operatorname{sgn}\left(\Delta X_{t i}\right)\left(1-\kappa_{t i}\right) \frac{d X_{t i}}{d \tau} d F(i)\right) f_{t} \tau_{t}^{*}\right]}{\mathbb{E}\left[\sum_{t=1}^{T} \beta^{t} \int\left[P_{t} \operatorname{sgn}\left(\Delta X_{t i}\right)\left(1-\kappa_{t i}\right)\right] \frac{d X_{t i}}{d \tau} d F(i)\right]}
$$

Hence, be defining:

$$
\omega_{t} \equiv \frac{\beta^{t} \int P_{t} \operatorname{sgn}\left(\Delta X_{t i}\right)\left(1-\kappa_{t i}\right) \frac{d X_{t i}}{d \tau} d F(i)}{\mathbb{E}\left[\sum_{t=1}^{T} \beta^{t} \int P_{t} \operatorname{sgn}\left(\Delta X_{t i}\right)\left(1-\kappa_{t i}\right) \frac{d X_{t i}}{d \tau} d F(i)\right]},
$$


we can finally write:

$$
\tau^{*}=\sum_{t=1}^{T} \mathbb{E}\left[\omega_{t} f_{t} \tau_{t}^{*}\right]
$$

It is easy to check that $\sum_{t=1}^{T} \mathbb{E}\left[\omega_{t}\right]=1$. Note that for this decomposition to be well defined, all denominators must be different from zero.

b) By defining $l_{t i} \equiv \frac{\operatorname{sgn}\left(\Delta X_{t i}\right) \frac{d X_{t i}}{d \tau}}{\int \operatorname{sgn}\left(\Delta X_{t i}\right) \frac{d X_{t i}}{d \tau} d F(i)}$, we can rewrite $f_{t}$ as:

$$
f_{t} \equiv \frac{1}{\int l_{t i}\left(1-\kappa_{t i}\right) d F(i)}
$$

where $\int l_{t i} d F(i)=1$. Hence, we interpret as the inverse of a weighted sum of all forward-looking distortions. If $\kappa_{t i}$ is constant across investors, we can write $f_{t}=\frac{1}{1-\kappa_{t i}}$.

Equation expression allows for an easy interpretation. When all investors are of the buy-and-hold type, $\int l_{t i}\left(1-\kappa_{t i}\right)=0$ and $f_{t}=1$. When most investors are permanent buyers (sellers), $\int l_{t i}\left(1-\kappa_{t i}\right)<1$ and $f_{t}>1$. When most investors are buyers-then-sellers, or viceversa, $\int l_{t i}\left(1-\kappa_{t i}\right)>1$ and $f_{t}<1$. This proves part $b$ ).

\section{Proposition 11. (Price volatility arguments revisited)}

a) Because now the planner respects individual beliefs, we can write the change in indirect utility for an investor $i$ as:

$$
\begin{gathered}
\frac{d V_{i}}{d \tau}=\mathbb{E}_{i}\left[\sum_{t=1}^{T} \beta^{t-1}\left(\xi_{t i} \frac{d X_{t i}}{d \tau} \tau-U_{i}^{\prime}\left(C_{t i}\right) \Delta X_{t i} \frac{d P_{t}}{d \tau}\right)\right] \\
\xi_{t i}=U_{i}^{\prime}\left(C_{t i}\right) P_{t}\left(1-\mathbb{E}_{t i}\left[\frac{\beta U_{i}^{\prime}\left(C_{t+1 i}\right)}{U_{i}^{\prime}\left(C_{t i}\right)} \frac{P_{t+1}}{P_{t}} \operatorname{sgn}\left(\Delta X_{t i}\right) \operatorname{sgn}\left(\Delta X_{t+1 i}\right)\right]\right)
\end{gathered}
$$

Note that in this case, the distortions in precautionary savings do not give rise to any wedge from the planner's perspective, as well as distorted beliefs for portfolio allocations. Hence, we can write the marginal change in social welfare as:

$$
\frac{d V}{d \tau}=\int \lambda_{i} \sum_{t=1}^{T} \beta^{t-1} \mathbb{E}\left[Z_{i}\left(\xi_{t i} \frac{d X_{t i}}{d \tau} \tau-U_{i}^{\prime}\left(C_{t i}\right) \Delta X_{t i} \frac{d P_{t}}{d \tau}\right)\right] d F(i)
$$

The results in the paper correspond to the $\beta=1$ case.

b) By solving for $\tau^{*}$ we find:

$$
\tau^{*}=\frac{\sum_{t=1}^{T} \beta^{t-1} \mathbb{E}\left[\int \lambda_{i} Z_{i} U_{i}^{\prime}\left(C_{t i}\right) \Delta X_{t i} d F(i) \frac{d P_{t}}{d \tau}\right]}{\sum_{t=1}^{T} \beta^{t-1} \mathbb{E}\left[\int \lambda_{i} Z_{i} \xi_{t i} \frac{d X_{t i}}{d \tau} d F(i)\right]}
$$

This concludes the proof of proposition 11. The following derivations correspond to the discussion in the text. First, we can write:

$$
\mathbb{E}\left[\int \lambda_{i} Z_{i} U_{i}^{\prime}\left(C_{t i}\right) \Delta X_{t i} d F(i) \frac{d P_{t}}{d \tau}\right]=\mathbb{E}\left[\zeta(\tau) \mathbb{E}_{F, \mathcal{T}}\left[\lambda_{i} Z_{i} U_{i}^{\prime}\left(C_{t i}\right) \Delta X_{t i}\right] \frac{d P_{t}}{d \tau}\right]
$$

We can also write:

$$
\begin{aligned}
\mathbb{E}_{F, \mathcal{T}}\left[\lambda_{i} Z_{i} U_{i}^{\prime}\left(C_{t i}\right) \Delta X_{t i}\right] & =\mathbb{E}_{F, \mathcal{T}}\left[\lambda_{i} Z_{t i} U_{i}^{\prime}\left(C_{t i}\right)\right] \mathbb{E}_{F, \mathcal{T}}\left[\Delta X_{t i}\right]-\operatorname{Cov}_{F, \mathcal{T}}\left[\lambda_{i} Z_{t i} U_{i}^{\prime}\left(C_{t i}\right), \Delta X_{t i}\right] \\
& =-\operatorname{Cov}_{F, \mathcal{T}}\left[\lambda_{i} Z_{t i} U_{i}^{\prime}\left(C_{t i}\right), \Delta X_{t i}\right]
\end{aligned}
$$


where I have used market clearing to derive the last line, since $\mathbb{E}_{F, \mathcal{T}}\left[\Delta X_{t i}\right]=0$. Hence:

$$
\mathbb{E}\left[\int \lambda_{i} Z_{i} U_{i}^{\prime}\left(C_{t i}\right) \Delta X_{t i} d F(i) \frac{d P_{t}}{d \tau}\right]=-\mathbb{E}\left[\zeta(\tau) \operatorname{Cov}_{F}\left[\lambda_{i} Z_{t i} U_{i}^{\prime}\left(C_{t i}\right), \Delta X_{t i}\right] \frac{d P_{t}}{d \tau}\right]
$$

Finally, an example of Pareto improvement is the following. Take a two agent economy. The marginal welfare change for every investor at $\tau=0$ is:

$$
\begin{gathered}
\frac{d V_{A}}{d \tau}=\mathbb{E}\left[\sum_{t=1}^{T}\left(-Z_{A} U_{A}^{\prime}\left(C_{t A}\right) \Delta X_{t A} \frac{d P_{t}}{d \tau}\right)\right] \\
\frac{d V_{B}}{d \tau}=\mathbb{E}[\sum_{t=1}^{T} \underbrace{\frac{Z_{B} U_{B}^{\prime}\left(C_{t B}\right)}{Z_{A} U_{A}^{\prime}\left(C_{t A}\right)}}_{m} Z_{A} U_{A}^{\prime}\left(C_{t A}\right) \Delta X_{t A} \frac{d P_{t}}{d \tau}]
\end{gathered}
$$

A Pareto improvement arises if both $\frac{d V_{A}}{d \tau}$ and $\frac{d V_{B}}{d \tau}$ are strictly positive. If $m=1$, we would reach a contradiction if we assumed that $\frac{d V_{A}}{d \tau}$ and $\frac{d V_{B}}{d \tau}$ are positive at the same time. However, when $m \neq 1$, depending on the patterns price changes it is completely possible to find a a strict Pareto improvement.

\section{Proofs: Section 7.1}

\section{Proposition 12. (Marginal effect on welfare/Optimal tax with production)}

a) The demand by investors for the risky asset is identical to the baseline model. The expression for the asset price in (39) now yields a demand curve for shares.

$$
P_{1}^{d}=\frac{\int_{i \in \mathcal{T}}\left(\frac{\mathbb{E}_{i}[D]}{\mathcal{A}_{i}}-A\left(\operatorname{Cov}\left[E_{2 i}, D\right]+\mathbb{V a r}[D] X_{0 i}\right)\right) d F(i)-A \mathbb{V a r}[D] S_{1 k}}{1+\tau \int_{i \in \mathcal{T}} \frac{\operatorname{sgn}\left(\Delta X_{1 i}\right)}{\mathcal{A}_{i}} d F(i)}
$$

The equilibrium price is now determined by the intersection of equation (39) and the supply curve, given by $P_{1}^{s}=\gamma_{1}+\gamma_{2} S_{1 k}$.

The marginal change in social welfare is given by:

$\frac{d V}{d \tau}=\int \lambda_{i} \mathbb{E}\left[U_{i}^{\prime}\left(W_{2 i}\right)\right]\left[\left(\mathbb{E}[D]-\mathbb{E}_{i}[D]+\operatorname{sgn}\left(\Delta X_{1 i}\right) P_{1} \tau\right) \frac{d X_{1 i}}{d \tau}-\Delta X_{1 i} \frac{d P_{1}}{d \tau}\right] d F(i)+\lambda_{k} \mathbb{E}\left[U_{k}^{\prime}\left(C_{2 k}\right)\right] \frac{d P_{1}}{d \tau} S_{1 k}$

Imposing assumption [NR], and using market clearing, that is, $\int \frac{d X_{1 i}}{d \tau} d F(i)=\frac{d S_{1 k}}{d \tau}$, we can write the marginal change in social welfare as:

$$
\frac{d V}{d \tau}=\int\left(\mathbb{E}[D]-\mathbb{E}_{i}[D]+\operatorname{sgn}\left(\Delta X_{1 i}\right) P_{1} \tau\right) \frac{d X_{1 i}}{d \tau} d F(i)
$$

This shows proposition 12a.

b) Solving for $\tau^{*}$ in the previous expression yields proposition $12 \mathrm{~b}$.

$$
\tau^{*}=\frac{\int\left(\frac{\mathbb{E}[D]-\mathbb{E}_{i}[D]}{P_{1}}\right) \frac{d X_{1 i}}{d \tau} d F(i)}{-\int \operatorname{sgn}\left(\Delta X_{1 i}\right) \frac{d X_{1 i}}{d \tau} d F(i)}
$$

The corollary to proposition 12 follows directly. 
We can re-write the numerator as:

$$
\begin{aligned}
\int\left(\mathbb{E}[D]-\mathbb{E}_{i}[D]\right) \frac{d X_{1 i}}{d \tau} d F(i) & =\zeta(\tau) \mathbb{E}_{F, \mathcal{T}}\left[\left(\mathbb{E}[D]-\mathbb{E}_{i}[D]\right) \frac{d X_{1 i}}{d \tau}\right] \\
& =\zeta(\tau)\left(\operatorname{Cov}_{F, \mathcal{T}}\left[\mathbb{E}[D]-\mathbb{E}_{i}[D], \frac{d X_{1 i}}{d \tau}\right]+\mathbb{E}_{F, \mathcal{T}}\left[\mathbb{E}[D]-\mathbb{E}_{i}[D]\right] \mathbb{E}_{F, \mathcal{T}}\left[\frac{d X_{1 i}}{d \tau}\right]\right) \\
& =\zeta(\tau)\left(-\operatorname{Cov}_{F, \mathcal{T}}\left[\mathbb{E}_{i}[D], \frac{d X_{1 i}}{d \tau}\right]+\left(\mathbb{E}[D]-\mathbb{E}_{F, \mathcal{T}}\left[\mathbb{E}_{i}[D]\right]\right) \frac{d S_{1 k}}{d \tau}\right)
\end{aligned}
$$

Where we define $\zeta(\tau) \equiv \int_{i \in \mathcal{T}} d F(i)$; this normalization by the number of active investors is necessary to use expectation and covariance operators. Using the fact that $\int_{i \in \mathcal{S}} \frac{d X_{1 i}}{d \tau} d F(i)=\frac{d S_{1 k}}{d \tau}-\int_{i \in \mathcal{B}} \frac{d X_{1 i}}{d \tau} d F(i)$, the denominator can be written as:

$$
\begin{aligned}
\int \operatorname{sgn}\left(\Delta X_{1 i}\right) \frac{d X_{1 i}}{d \tau} d F(i) & =\int_{i \in \mathcal{B}} \frac{d X_{1 i}}{d \tau} d F(i)-\int_{i \in \mathcal{S}} \frac{d X_{1 i}}{d \tau} d F(i)=2 \int_{i \in \mathcal{B}} \frac{d X_{1 i}}{d \tau} d F(i)-\frac{d S_{1 k}}{d \tau} \\
& =2 \zeta(\tau) \int_{i \in \mathcal{B}, \mathcal{T}} \frac{d X_{1 i}}{d \tau} d F(i)-\frac{d S_{1 k}}{d \tau}
\end{aligned}
$$

By substituting and rearranging the optimal tax formula, we find that:

$$
\begin{aligned}
& \tau^{*}= \frac{\zeta(\tau) \operatorname{Cov}_{F, \mathcal{T}}\left[\frac{\mathbb{E}_{i}[D]}{P_{1}}, \frac{d X_{1 i}}{d \tau}\right]}{2 \zeta(\tau) \int_{i \in \mathcal{B}, \mathcal{T}} \frac{d X_{1 i}}{d \tau} d F(i)-\frac{d S_{1 k}}{d \tau}}+\frac{\zeta(\tau)\left(\frac{\mathbb{E}[D]-\mathbb{E}_{F, \mathcal{T}}\left[\mathbb{E}_{i}[D]\right]}{P_{1}}\right)\left(-\frac{d S_{1 k}}{d \tau}\right)}{2 \zeta(\tau) \int_{i \in \mathcal{B}, \mathcal{T}} \frac{d X_{1 i}}{d \tau} d F(i)-\frac{d S_{1 k}}{d \tau}} \\
& \tau^{*}= \underbrace{\frac{2 \zeta(\tau) \int_{i \in B, \mathcal{T}} \frac{d X_{1 i}}{d \tau} d F(i)}{2 \zeta(\tau) \int_{i \in \mathcal{B}, \mathcal{T}} \frac{d X_{1 i}}{d \tau} d F(i)+\left(-\frac{d S_{1 k}}{d \tau}\right)}}_{\equiv \omega} \underbrace{\left(\frac{\operatorname{Cov}_{F, \mathcal{T}}\left[\frac{\mathbb{E}_{i}[D]}{P_{1}}, \frac{d X_{1 i}}{d \tau}\right]}{2 \int_{i \in \mathcal{B}, \mathcal{T}} \frac{d X_{1 i} d F(i)}{d \tau} d F\left(i S_{1 k}\right.}\right)}_{\equiv \tau_{\text {exchange }}^{*}}+ \\
&+\underbrace{\left(2 \zeta(\tau) \int_{i \in \mathcal{B}, \mathcal{T}} \frac{d X_{1 i}}{d \tau} d F(i)+\left(-\frac{d S_{1 k}}{d \tau}\right)\right)}_{\equiv 1-\omega} \zeta(\tau) \underbrace{\left(\frac{\mathbb{E}[D]-\mathbb{E}_{F, \mathcal{T}}\left[\mathbb{E}_{i}[D]\right]}{P_{1}}\right)}_{\tau_{\text {production }}^{*}}
\end{aligned}
$$

Therefore, the optimal tax can be seen as a linear combination between the optimal tax in an exchange economy and the excess return of the risky, that is:

$$
\tau^{*}=\omega \tau_{\text {exchange }}^{*}+(1-\omega) \zeta(\tau) \tau_{\text {production }}^{*}
$$

In equation (35), $v$ is defined by: $v \equiv \frac{\zeta(\tau)}{1+\tau \int_{i \in \mathcal{T}} \operatorname{sgn}\left(\Delta X_{1 i}\right) d F(i)}$.

\section{Proofs: Section 7.2}

Equilibrium definition (Hayekian production) Under assumption (5), given a realization of $\theta$, the definition of equilibrium is Walrasian. Thus, a competitive equilibrium with taxes is defined as a portfolio allocation $X_{1 i}$ for both informed and uninformed investors, an investment decision $k^{*}$, a price $P_{1}$ and set of lump-sum transfers $T_{1 i}$ such that: informed investors maximize utility in $X_{1 i}$ given $\theta$ subject to their budget constraint (3), yielding a demand function which only depends on $\theta$ and $P_{1}$, without taking into account the effect of their trading in production and assuming that $k^{*}=\mathbb{E}[\theta]=0$; uninformed investors maximize utility in $X_{1 i}$, subject to their budget constraint, yielding a demand function which only depends on $P_{1}$ and assuming that $k^{*}=\mathbb{E}[\theta]=0$; the manager of the firm maximizes profit per share, conditioning its choice of $k^{*}$ on the equilibrium price; the price $P_{1}$ is such the market for the risky asset clears, that is $\sum_{i} X_{1 i}=Q$ and tax revenues are rebated in a lump-sum fashion to investors, that is $\int T_{1 i} d F(i)=\tau\left|P_{1}\right| \int\left|\Delta X_{1 i}\right| d F(i)$. 


\section{Lemma 3. (Inaction thresholds)}

a) Under assumption 6, for a given price $P_{1}$, an informed investor decides not to trade, which effectively implies that there is no-trade in equilibrium, when

$$
\frac{\mathbb{E}[D]+\theta-\delta \mathbb{V a r}[\theta]-P_{1}(1+\tau)}{A_{I} \mathbb{V} \operatorname{Var}[D]}<Q \quad \text { or } \quad \frac{\mathbb{E}[D]+\theta-\delta \mathbb{V a r}[\theta]-P_{1}(1-\tau)}{A_{I} \mathbb{V} \operatorname{Var}[D]}>Q
$$

By substituting the equilibrium price $P_{1}=\mathbb{E}[D]+\frac{\theta}{2}-Q A_{I} \mathbb{V}$ ar $[D]$ into both inequalities and solving for $\theta$, the no-trade region is characterized by:

$$
-\frac{\tau}{1+\tau} 2\left(\mathbb{E}[D]-Q A_{I} \mathbb{V} \text { ar }[D]\right)<\theta<\frac{\tau}{1-\tau} 2\left(\mathbb{E}[D]-Q A_{I} \mathbb{V} \text { ar }[D]\right)
$$

This proves part a) of lemma 3 .

b) The derivatives with respect to $\tau$ of the threshold function are given by:

$$
\frac{d \underline{\theta}(\tau)}{d \tau}=-\frac{2\left(\mathbb{E}[D]-Q A_{I} \mathbb{V} \operatorname{ar}[D]\right)}{(1+\tau)^{2}}<0 \quad \frac{d \bar{\theta}(\tau)}{d \tau}=\frac{2\left(\mathbb{E}[D]-Q A_{I} \mathbb{V} a r[D]\right)}{(1-\tau)^{2}}>0
$$

Which under assumption 6 proves part b). Note that both derivatives are strictly positive even when $\tau=0$. This implies that an infinitesimal tax creates an inaction region of positive measure.

\section{Lemma 4. (Welfare gap)}

I define $V^{T}(\underline{\theta}) \equiv \lim _{\theta \rightarrow \theta^{-}} V(\theta)$ and $V^{N T}(\underline{\theta}) \equiv \lim _{\theta \rightarrow \theta^{+}} V(\theta)$. And $V^{T}(\bar{\theta}) \equiv \lim _{\theta \rightarrow \theta^{+}} V(\theta)$ and $V^{N T}(\bar{\theta}) \equiv \lim _{\theta \rightarrow \underline{\theta}^{-}} V(\theta)$. For a given $\theta=\{\underline{\theta}, \bar{\theta}\}$, we can write the difference of social welfare when the informed investors trade with respect to the case when they do not trade:

$$
\begin{aligned}
V^{T}(\theta)-V^{N T}(\theta) & =\sum_{i} \pi_{i} \lambda_{i}\left(-e^{-A_{i}\left((\mathbb{E}[D]+\theta)+\delta \theta^{2} X_{0 i}-\frac{A_{i}}{2} \operatorname{Var}[D] X_{0 i}^{2}\right)}-\left(-e^{-A_{i}\left((\mathbb{E}[D]+\theta) X_{0 i}-\frac{A_{i}}{2} \mathbb{V a r}[D] X_{0 i}^{2}\right)}\right)\right) \\
& =\sum_{i} \pi_{i} \lambda_{i}\left(-e^{-A_{i}\left((\mathbb{E}[D]+\theta) X_{0 i}-\frac{A_{i}}{2} \operatorname{Var}[D] X_{0 i}^{2}\right)}\left(e^{-A_{i} \delta \theta^{2} X_{0 i}}-1\right)\right) \\
& =\sum_{i} \pi_{i} \lambda_{i} \mathbb{E}\left[U^{\prime}\left(W_{2 i}\left(X_{0 i}\right)\right) \mid \theta\right] \frac{1}{A_{i}}\left(e^{-A_{i} \delta \theta^{2} X_{0 i}}-1\right) \\
& =\sum_{i} \pi_{i} \lambda_{i} \mathbb{E}\left[U\left(W_{2 i}\left(X_{0 i}\right)\right) \mid \theta\right] \frac{1}{A_{i}}\left(1-e^{-A_{i} \delta \theta^{2} X_{0 i}}\right)
\end{aligned}
$$

For the last line, I have used the fact that CARA utility satisfies the property $-A_{i} U_{i}(x)=U_{i}^{\prime}(x)$. This property hence implies that $-\mathbb{E}\left[U_{i}\left(W_{2 i}\left(X_{0 i}\right)\right) \mid \theta\right]=\frac{1}{A_{i}} \mathbb{E}\left[U_{i}^{\prime}\left(W_{2 i}\left(X_{0 i}\right)\right) \mid \theta\right]$. Therefore, using assumption (7), we can approximate $V^{T}(\theta)-V^{N T}(\theta)$ up to a first order around $A_{i}=0$ or $\delta=0$ in the following way:

$$
V^{T}(\theta)-V^{N T}(\theta)=\sum_{i} \pi_{i}\left(1-e^{-A_{i} \delta \theta^{2} X_{0 i}}\right) \approx \sum_{i} \pi_{i} \delta \theta^{2} X_{0 i}=\delta \theta^{2} Q
$$

where I have used market clearing as $\sum_{i} \pi_{i} X_{0 i}=Q$.

\section{Proposition 13. (Marginal effect on welfare with information diffusion)}

a) I use $\mathbb{E}_{\theta \in \Theta}\left[\left.\frac{d V}{d \tau}\right|_{\theta}\right]$ to represent:

$$
\left.\mathbb{E}_{\theta \in \Theta}\left[\left.\frac{d V}{d \tau}\right|_{\theta}\right] \equiv \int_{-\infty}^{\underline{\theta}(\tau)} \frac{d V}{d \tau}\right|_{\theta} \phi_{\mathbb{V a r}[\theta]}(\theta) d \theta+\left.\int_{\bar{\theta}(\tau)}^{\infty} \frac{d V}{d \tau}\right|_{\theta} \phi_{\mathbb{V a r}[\theta]}(\theta) d \theta
$$


where $\left.\frac{d V}{d \tau}\right|_{\theta}=\left.\int \mathbb{E}\left[U_{i}^{\prime}\left(W_{2 i}\right) \mid \theta\right] \frac{d \tilde{V}_{i}}{d \tau}\right|_{\theta} d F(i)$, and where $\frac{d \tilde{V}_{i}}{d \tau}$ is given by:

$$
\begin{aligned}
\frac{d \tilde{V}_{i}}{d \tau}=\mathbb{E}_{\theta}\left[\left(\mathbb{E}[\Pi]-P_{1}-A_{i} X_{1 i} \mathbb{V a r}[D]\right) \frac{d X_{1 i}}{d \tau}-\Delta X_{1 i} \frac{d P_{1}}{d \tau}\right] \\
=\mathbb{E}_{\theta}\left[(\mathbb{E}[\Pi]-P_{1}-\underbrace{\frac{\mathbb{V} a r[D]}{\mathbb{V a r}[D+\theta]}}_{r_{i}}\left(\mathbb{E}\left[\Pi_{i}\right]-P_{1}\left(1+\operatorname{sgn}\left(\Delta X_{1 i}\right) \operatorname{sgn}\left(\Delta X_{1 i}\right) \tau\right)\right)) \frac{d X_{1 i}}{d \tau}-\Delta X_{1 i} \frac{d P_{1}}{d \tau}\right]
\end{aligned}
$$

Differentiating social welfare, using lemma 3 and applying Leibniz rule for differentiation, it follows that:

$$
\frac{d V}{d \tau}=\mathbb{E}_{\theta \in \Theta}\left[\left.\frac{d V}{d \tau}\right|_{\theta}\right]+\left(V^{T}(\underline{\theta})-V^{N T}(\underline{\theta})\right) \phi_{\mathbb{V} a r[\theta]}(\underline{\theta}) \frac{d \underline{\theta}(\tau)}{d \tau}+\left(V^{N T}(\bar{\theta})-V^{T}(\bar{\theta})\right) \phi_{\mathbb{V} a r[\theta]}(\bar{\theta}) \frac{d \bar{\theta}(\tau)}{d \tau},
$$

where $V^{T}(\underline{\theta})-V^{N T}(\underline{\theta})$ and $V^{N T}(\bar{\theta})-V^{T}(\bar{\theta})$ are defined above. And I define $\Psi(\tau)$ by:

$$
\Psi(\tau) \equiv\left(V^{T}(\underline{\theta})-V^{N T}(\underline{\theta})\right) \phi_{\mathbb{V a r}[\theta]}(\underline{\theta}) \frac{d \underline{\theta}(\tau)}{d \tau}+\left(V^{N T}(\bar{\theta})-V^{T}(\bar{\theta})\right) \phi_{\mathbb{V} a r[\theta]}(\bar{\theta}) \frac{d \bar{\theta}(\tau)}{d \tau}
$$

b) When $\tau=0, \Psi(0)$ can be written as:

$$
\Psi(0)=\underbrace{\left(V^{T}(\underline{\theta})-V^{N T}(\underline{\theta})\right)}_{>0} \phi_{\mathbb{V a r}[\theta]}(\underline{\theta}) \underbrace{\frac{d \underline{\theta}(0)}{d \tau}}_{<0}+\underbrace{\left(V^{N T}(\bar{\theta})-V^{T}(\bar{\theta})\right)}_{<0} \phi_{\mathbb{V a r}[\theta]}(\bar{\theta}) \underbrace{\frac{d \bar{\theta}(0)}{d \tau}}_{>0}<0
$$

Therefore, combining the results from lemmas 3 and 4 , and using the fact that $\phi_{\mathbb{V} a r[\theta]}(\cdot)$ is strictly positive, we can conclude that $\Psi(0)<0$.

\section{Proposition 14. (Optimal tax with information diffusion)}

By solving for $\tau^{*}$ in the expression for $\frac{d V}{d \tau}$, we find:

$$
\tau^{*}=\frac{\mathbb{E}_{\theta}\left[\sum_{i} \pi_{i} r_{i} \mathbb{E}_{i}[D] \frac{d X_{1 i}}{d \tau}\right]+\left(V^{T}(\underline{\theta})-V^{N T}(\underline{\theta})\right) \phi_{\mathbb{V a r}[\theta]}(\underline{\theta}) \frac{d \underline{\theta}(\tau)}{d \tau}+\left(V^{N T}(\bar{\theta})-V^{T}(\bar{\theta})\right) \phi_{\mathbb{V a r}[\theta]}(\bar{\theta}) \frac{d \bar{\theta}(\tau)}{d \tau}}{P_{1} \mathbb{E}_{\theta}\left[\sum_{i} \pi_{i} r_{i}\left(1+\operatorname{sgn}\left(\Delta X_{1 i}\right) \operatorname{sgn}\left(P_{1}\right) \frac{d X_{1 i}}{d \tau}\right)\right]}
$$

Using lemma 4, to substitute $V^{T}(\theta)-V^{N T}(\theta)$ for $\delta \theta^{2} Q$, we can write:

$$
\tau^{*}=\frac{\mathbb{E}_{\theta}\left[\sum_{i} \pi_{i} r_{i} \mathbb{E}_{i}[D] \frac{d X_{1 i}}{d \tau}\right]+\delta Q\left[(\underline{\theta})^{2} \phi_{\mathbb{V a r}[\theta]}(\underline{\theta}) \frac{d \theta}{d \tau}+(\bar{\theta})^{2} \phi_{\mathbb{V a r}[\theta]}(\bar{\theta}) \frac{d \bar{\theta}}{d \tau}\right]}{P_{1} \mathbb{E}_{\theta}\left[\sum_{i} \pi_{i} r_{i}\left(1+\operatorname{sgn}\left(\Delta X_{1 i}\right) \operatorname{sgn}\left(P_{1}\right) \frac{d X_{1 i}}{d \tau}\right)\right]}
$$

So we can define $\tau^{*}=\tau_{\text {exchange }}^{*}+\tau_{\text {information }}^{*}$ :

$$
\begin{aligned}
\tau_{\text {exchange }}^{*} & \equiv \frac{\mathbb{E}_{\theta}\left[\sum_{i} \pi_{i} r_{i} \mathbb{E}_{i}[D] \frac{d X_{1 i}}{d \tau}\right]}{P_{1} \mathbb{E}_{\theta}\left[\sum_{i} \pi_{i} r_{i}\left(1+\operatorname{sgn}\left(\Delta X_{1 i}\right) \operatorname{sgn}\left(P_{1}\right) \frac{d X_{1 i}}{d \tau}\right)\right]} \\
\tau_{\text {information }}^{*} & \equiv \frac{\delta Q\left[(\underline{\theta})^{2} \phi_{\mathbb{V} a r[\theta]}(\underline{\theta}) \frac{d \theta}{d \tau}+(\bar{\theta})^{2} \phi_{\mathbb{V a r}[\theta]}(\bar{\theta}) \frac{d \bar{\theta}}{d \tau}\right]}{P_{1} \mathbb{E}_{\theta}\left[\sum_{i} \pi_{i} r_{i}\left(1+\operatorname{sgn}\left(\Delta X_{1 i}\right) \operatorname{sgn}\left(P_{1}\right) \frac{d X_{1 i}}{d \tau}\right)\right]}
\end{aligned}
$$




\section{Appendix B: Additional results and extensions}

\section{A Remarks about modeling choices}

\section{Baseline model}

- Sources of belief disagreement: because it is not crucial for the results, I do not model explicitly the sources of disagreement. Disagreement naturally arises when investors have different learning mechanisms. For instance, they may be overconfident about some pieces of information, as in Scheinkman and Xiong (2003), but they could be subject to alternative behavioral biases. See, for instance, the discussion in Shleifer (2000). Under the "common prior" assumption - see Morris (1995) - there exists a one-to-one mapping between behavioral departures from Bayesian updating in processing information and heterogeneous priors/belief disagreement.

- Instruments: as shown in subsection 5.5, because of the second-best nature of the problem addressed, additional instruments are in general welfare improving. For instance, short-sale constraints and borrowing constraints are potentially useful policy instruments, especially in the model with production. Transaction taxes which phase out at a given horizon are also natural policies to use. Allowing some groups of investors to be exempt from paying the tax could be also welfare improving in theory. More instruments introduce the practical concern of increasing the informational requirements for the planner.

- Untaxed riskless asset: the fact that trading in the riskless asset is not subject to a tax does not modify the main conclusions of the paper. Taxing equally all tradable assets in a portfolio choice problem acts as a lump-sum tax, since every investor is forced to purchase at least an asset to "store" his wealth and all assets face an equivalent tax. With an additional margin of adjustment (e.g., consumption), a trading tax would have an additional effect similar to classic capital taxation rather than trading distortions.

- Tax definition: taxes could alternatively be imposed on the total amount traded over a period of time and not on the per trade amount. The more agent-dependent a tax is defined, as opposed to transaction-dependent, the larger the incentive to get around it. An individual specific tax can be overcome at least in two ways. First, investors could create multiple entities and trade with each of them until facing the minimum marginal tax. Second, they could distribute trades among periods to minimize tax liabilities. See Campbell and Froot (1994) for related arguments.

- Risk neutrality: welfare losses in this paper arise from the curvature of the utility function. When all investors are risk neutral, any portfolio allocation or production decision yields the same social welfare for the planner. For instance, the planner of this paper would suggest that all allocations in Scheinkman and Xiong (2003) are equally efficient, independently of the level of overconfidence.

- Exogenous markets: I assume throughout that the set of assets traded/active markets is exogenously given. Nonetheless, modifying transaction taxes may dramatically affect the set of assets traded. In subsection 5.2, we can think that the planner is endogenously taking into account the possibility that in some market trade ceases to occur. There is scope for better understanding how transaction taxes may shut down specific markets or create new ones.

\section{Dynamic model}

- Finite horizon: by working on a finite horizon economy, I avoid issues related to survival and degeneracy of long-run wealth distributions.

- OLG: often used overlapping generations models would not be adequate in this context, since they preclude the existence of buy-and-hold and buy-and-buy investors. 
- Intermediate consumption choice vs. final consumption: allowing investors to have intermediate consumption simplifies the characterization of the optimal tax. This allows for the use of variational arguments to characterize Euler equations.

- Exogenous interest rates: the results extend directly to the case of endogenous interest rates.

\section{Walrasian production}

- Single versus multiple sectors: in the paper, welfare distortions arise because the amount of aggregate risk held by investors is distorted. The results extend naturally to multiple sectors. In that case, the same logic applies to the amount invested across sectors. When investors are wrong on average about the payoffs of different sectors, some sectors happen to receive more investment and some sectors less. The optimal tax weights the distortions according to the relative investment sensitivities across sectors.

- Producers are not allowed to hold the risky asset: all results are robust to allowing producers to hold the risky asset. This assumption only simplifies market clearing.

\section{B Differentiability and convexity properties}

I only analyze explicitly the baseline model; a similar logic applies to the rest of the extensions.

Differentiability From equation (7) it is straightforward to show that the equilibrium price $P_{1}$ is continuous in $\tau$. As shown in the proof of lemma 1, for the values of $\tau$ such that $P_{1}$ is differentiable we have that $\frac{d P_{1}}{d \tau}=-\frac{P_{1} \int_{i \in \mathcal{T}} \frac{\operatorname{sgn}\left(\Delta X_{1 i}\right)}{A_{i}} d F(i)}{\int_{i \in \mathcal{T}} \frac{1+\operatorname{sgn}\left(\Delta X_{1 i}\right) \tau}{A_{i}} d F(i)}$. Hence, when the distribution $F$ of investors is continuous across all dimensions of heterogeneity, $\frac{d P_{1}}{d \tau}$ is continuous, since both numerator and denominator change smoothly with $\tau$, which implies that $P_{1}$ is differentiable. When $F$ has mass points, $\frac{d P_{1}}{d \tau}$ is discontinuous for those values of $\tau$ in which a positive measure of investors becomes inactive, since the left and right limits to $\int_{i \in \mathcal{T}} \frac{\operatorname{sgn}\left(\Delta X_{1 i}\right)}{A_{i}} d F(i)$ differ at that value of $\tau$. Because I have restricted the distribution of investors $F$ to be continuous, discrete or a mixture of both, $P_{1}$ is differentiable almost everywhere.

Because $X_{1 i}$ is continuous in $\tau$ and $P_{1}$, the equilibrium allocations inherit the differentiability

properties of $\frac{d P_{1}}{d \tau}$. Hence, social welfare $V(\tau)$ also inherits the differentiability properties of $\frac{d P_{1}}{d \tau}$. Throughout the paper I implicitly assume that it is never the case that the planner's problem reaches its optimum at a point of non-differentiability. A sufficient condition for this to hold is that the distribution of investors is continuous - I use a discrete number of agents in the simulations only for numerical tractability.

Summing up: when the distribution of investors $F$ is continuous, $P_{1}, X_{1 i}$ and $V$ are differentiable. When $F$ has mass points, there is a finite number of values for $\tau$ at which $P_{1}, X_{1 i}$ and $V$ are nondifferentiable.

Convexity In general, there is no guarantee that the planner's problem on $\tau$ is convex for a general distribution $F$. The non-convexity of the planner's problem is due to changes in the extensive margin, that is, in the composition of marginal investors for different values of $\tau$. In this section, I explore some properties of the problem related to non-convexities assuming that [NR] holds.

First, note that social welfare $V$ is continuous in $\tau$ and that, for $\tau$ sufficiently large, social welfare is constant, because investors cease to trade and their welfare corresponds to their autarky level, unless we are in the case in which, even when $\tau=\bar{\tau}$, some investors still decide to trade. In any case, because $\tau$ is bounded in $\tau \in[\underline{\tau}, \bar{\tau}]$, the existence of a maximum is guaranteed. We can show that the planner's 
problem is (locally) convex in all regions in which there are no extensive margin changes. In general, we can write $\frac{d V}{d \tau}$ as:

$$
\begin{aligned}
\frac{d V}{d \tau} & =\int\left[-\mathbb{E}_{i}[D]+\operatorname{sgn}\left(\Delta X_{1 i}\right) P_{1} \tau\right] \frac{d X_{1 i}}{d \tau} d F(i) \\
& =-\int \mathbb{E}_{i}[D] \frac{d X_{1 i}}{d \tau} d F(i)+2 P_{1} \tau \int_{i \in \mathcal{B}} \frac{d X_{1 i}}{d \tau} d F(i) \\
& =\frac{-P_{1}}{\operatorname{Var}[D]}\left[-\int \mathbb{E}_{i}[D] \frac{\operatorname{sgn}\left(\Delta X_{1 i}\right)}{A_{i}} \varepsilon_{i} d F(i)+2 P_{1} \tau \varepsilon_{\mathcal{B}} \int_{i \in \mathcal{B}} \frac{1}{A_{i}} d F(i)\right],
\end{aligned}
$$

where I have used the result from lemma 1 that $\frac{d X_{1 i}}{d \tau}=\frac{\partial X_{1 i}}{\partial \tau} \varepsilon_{i}$ and market clearing. Hence, as long as there are no extensive margin changes, the sign of $\frac{d^{2} V}{d \tau^{2}}$ is only determined by $\frac{d\left(-P_{1} \tau\right)}{d \tau}$. Therefore, for the problem to be convex, it is sufficient to show that $\frac{d\left(P_{1} \tau\right)}{d \tau}>0$, which is always the case because:

$$
\frac{d\left(P_{1} \tau\right)}{d \tau}=P_{1}\left(1-\frac{\tau \int \frac{\operatorname{sgn}\left(\Delta X_{1 i}\right)}{A_{i}} d F(i)}{\int \frac{1+\operatorname{sgn}\left(\Delta X_{1 i}\right) \tau}{A_{i}} d F(i)}\right)>0
$$

This result creates an important asymmetry between the $\tau>0$ and the $\tau \leq 0$ region. In the $\tau \leq 0$ region, social welfare is always convex because there are no extensive margin changes (buyers remain buyers for any level of the subsidy - the same applies to sellers). This result guarantees that when [OBPS] holds, the optimal tax has to be positive. When $\tau>0$, we would have to impose restrictions on the distribution of investors to find a unique maximum. Following this logic, even if [OBPS] does not hold, we can only guarantee that there will be a local maximum in the $\tau \leq 0$ region, but the global maximum may well entail $\tau^{*}>0$ - see the following example.

In figure A.1, I provide an example of a very non-convex problem. In this case there are 6 investors, with the the following characteristics:

\begin{tabular}{|c|c|c|c|c|c|c|}
\hline & $\pi_{1}=0.2$ & $\pi_{2}=0.2$ & $\pi_{3}=0.2$ & $\pi_{4}=0.2$ & $\pi_{5}=0.1$ & $\pi_{6}=0.1$ \\
\hline$A_{i}$ & 2 & 2 & 1 & 1 & 1 & 1 \\
\hline $\operatorname{Cov}\left[E_{2 i}, D\right]$ & -20 & 20 & 50 & -50 & 0 & 0 \\
\hline $\mathbb{E}_{i}[D]$ & 100 & 100 & 140 & 60 & 161 & 39 \\
\hline
\end{tabular}

Table 5: Parameters Figure A.1

All investors agree on the variance of the asset $\operatorname{Var}[D]=16$. The choice of values is very extreme. It simply illustrates clearly how nonconvexities may arise. In this particular example, [OBPS] does not hold, but the optimal tax happens to be positive. We see also in the figure the result just shown that, when there are no extensive margin changes, social welfare is convex. 

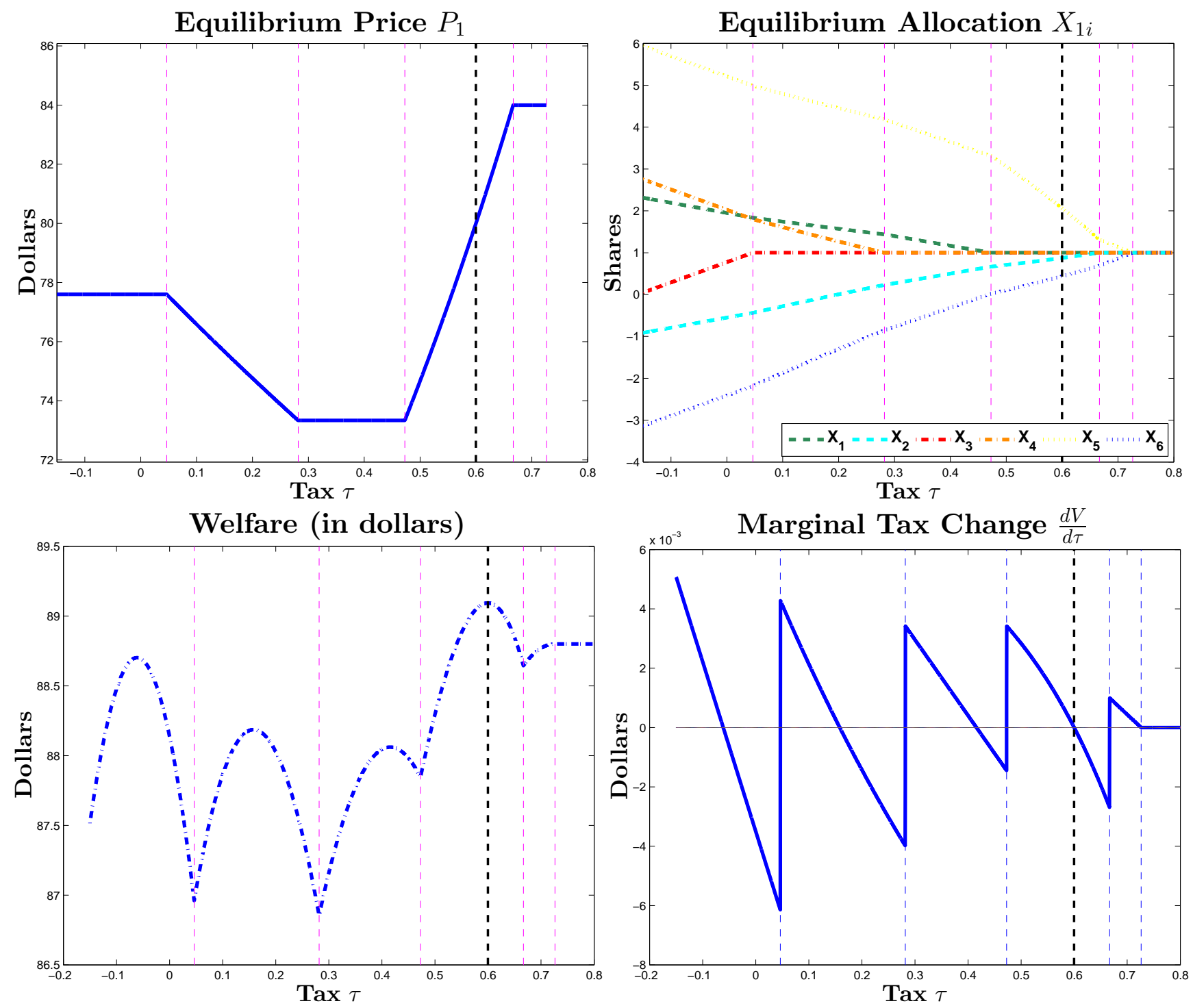

Figure A.1: Understanding non-convexities

The top left plot shows the equilibrium price and the upper right plot shows the equilibrium levels of trade for each investor. The bottom left plot shows social welfare in dollars and the bottom right one shows the derivative of this function $\frac{d V}{d \tau}$. The vertical purple dashed lines represent tax levels at which at least one of the investors stops trading. The vertical black dashed line represents the optimal tax level.

Summing up: extensive margin changes may generate non-convexities in the planner's problem. They have an important economic interpretation. Intuitively, a planner should always think whether a tax increase improves social welfare from the perspective of the marginal investors. For instance, if a group of fundamental investors have stopped to trade at a given tax rate, the planner would have further incentives to increase the tax if the only remaining investors trade for non-fundamental reasons. On the contrary, if many non-fundamental investors stop trading at a given tax rate, any marginal tax increase would generate substantial welfare losses. This logic applies directly to the case of high frequency investors, which become instantly inframarginal for any level of $\tau$.

\section{Tax on the number of shares}

I assume in the baseline model that the tax is levied on the dollar value of a trade rather than on the number of shares traded to prevent investors from circumvent it by varying the effective number of 
shares traded - through a reverse split. All results apply to taxes that depend on the number of shares with minor modifications.

When $P_{1}$ is exactly zero, a tax based on the dollar volume of the transaction is ineffective. However, a tax based on the number of shares traded $\left|\Delta X_{1 i}\right|$ can be introduced to effectively tax the notional value of the contract. I extend here propositions 1 and 2 to the case of taxes levied on the number of shares traded. In this case, the distinction between buyers and sellers is somewhat arbitrary, giving support to the idea that both sides of the market should face the same tax.

In the trade region, the optimal portfolio choice of an investor can be written as: $X_{1 i}=$ $\frac{\mathbb{E}_{i}[D]-A_{i} \operatorname{Cov}\left[E_{2 i}, D\right]-P_{1}-\operatorname{sgn}\left(\Delta X_{1 i}\right) \tau}{A_{i} \mathbb{V a r}[D]}$. The equilibrium price becomes:

$$
P_{1}=\int_{i \in \mathcal{T}}\left(\frac{\mathbb{E}_{i}[D]}{\mathcal{A}_{i}}-A\left(\operatorname{Cov}\left[E_{2 i}, D\right]-\mathbb{V} a r[D] X_{0 i}\right)-\frac{\operatorname{sgn}\left(\Delta X_{1 i}\right)}{\mathcal{A}_{i}} \tau\right) d F(i)
$$

The price corrections is now additive rather than multiplicative. The equivalent to equation (11) is $\frac{d V}{d \tau}=\int\left[-\mathbb{E}_{i}[D]+\operatorname{sgn}\left(\Delta X_{1 i}\right) \tau\right] \frac{d X_{1 i}}{d \tau} d F(i)$. The equivalent to equation (13) is now:

$$
\tau^{*}=\frac{\int \mathbb{E}_{i}[D] \frac{d X_{1 i}}{d \tau} d F(i)}{\int \operatorname{sgn}\left(\Delta X_{1 i}\right) \frac{d X_{1 i}}{d \tau} d F(i)}
$$

This shows that optimal taxes in the paper are written in terms of returns because they are levied on the dollar value of the transaction. When they are levied on the number of shares, the dispersion in expected payoffs rather than the dispersion in expected returns becomes the welfare relevant variable.

\section{Interest rate $R \neq 1$}

When $R \neq 1$, the equivalent to equation (11) is $\frac{d V}{d \tau}=\int\left[-\mathbb{E}_{i}[D]+\operatorname{sgn}\left(\Delta X_{1 i}\right) R P_{1} \tau\right] \frac{d X_{1 i}}{d \tau} d F(i)$. The equivalent to equation (13) is $\tau^{*}=\frac{1}{R} \frac{\int \mathbb{E}_{i}[D] \frac{d X_{1 i}}{d \tau} d F(i)}{\int \operatorname{sgn}\left(\Delta X_{1 i}\right) \frac{d X_{1 i}}{d \tau} d F(i)}$. In general, all tax formulas are divided by $R$. Intuitively, because all agents are marginal with respect to the riskless asset, the tax needs to be normalized by the marginal utility of wealth at $t=0$, which is given by $R$ since that is the marginal rate of return that all investors face. When $R$ is higher, a lower (dollar) tax is required to distort agents portfolio in the same degree.

\section{E Parametric belief distribution}

Starting from equation (14), it is possible to write the optimal tax as a function of moments of the distribution of beliefs, once a parametric distribution is chosen. For simplicity, I assume that agents are fully symmetric and that they only trade due to disagreement.

Assume that equation (14) holds and that the distribution of beliefs for a fraction $\theta$ of the investors is a normally distributed with mean $\mu$ and variance $\sigma^{2}$. All remaining $1-\theta$ investors belief that the expectation of the dividend process equals $\mu$. Note that an investor with belief $x$ only trades in this case when the tax is lower than the absolute value of $\frac{x-\mu}{P_{1}}$. The optimal tax is thus given by the following equation:

$$
\tau^{*}=\theta \frac{\sigma}{P_{1}} \Lambda\left(\frac{\tau^{*}}{\frac{\sigma}{P_{1}}}\right),
$$

where $\Lambda(\cdot)$ denotes the hazard rate of the normal. It can be easily shown that the value of the tax is an increasing function of $\sigma$. Intuitively, the larger the belief dispersion, the larger the magnitude of nonfundamental trading and the larger the need for a corrective tax. Similar derivations can be done with distributions that truncate easily, like Pareto or Exponential distributions. 


\section{F Theoretical justification for assumption [OBPS]}

Assumption [OBPS] directly determines the sign of the optimal tax. Here I provide a purely theoretical argument supporting assumption [OBPS]. Using the result from lemma 1 that shows that $\frac{d X_{1 i}}{d \tau}=\frac{\partial X_{1 i}}{\partial \tau} \varepsilon_{i}$, where $\varepsilon_{i}>0$, we can write:

$$
\begin{aligned}
\operatorname{Cov}_{F}\left[\mathbb{E}_{i}[D], \frac{\left.d X_{1 i}\right]}{d \tau}\right] & =\operatorname{Cov}_{F}\left[\mathbb{E}_{i}[D], \frac{\partial X_{1 i}}{\partial \tau} \varepsilon_{i}\right] \\
& =\frac{-P_{1}}{A \mathbb{V a r}[D]} \operatorname{Cov}_{F}\left[\mathbb{E}_{i}[D], \operatorname{sgn}\left(\Delta X_{1 i}\right) \varepsilon_{i}\right] \\
& =\frac{-P_{1}}{A \mathbb{V a r}[D]} \operatorname{Cov}_{F}\left[\mathbb{E}_{i}[D], \operatorname{sgn}\left(\frac{\mathbb{E}_{i}[D]-A \operatorname{Cov}\left[E_{2 i}, D\right]-P_{1}\left(1+\operatorname{sgn}\left(\Delta X_{1 i}\right) \tau\right)}{A \mathbb{V a r}[D]}-X_{0 i}\right) \varepsilon_{i}\right]
\end{aligned}
$$

The argument can be made from equation (40): as long as fundamental trading needs are orthogonal to investors' beliefs, we expect [OBPS] to hold. The term $\mathbb{E}_{i}[D]$ appears on both random variables that form part of the covariance. If the rest of the terms of $\operatorname{sgn}\left(\Delta X_{1 i}\right)$ were constant for all $i-$ that would correspond to the case when all trading is driven by disagreement, the covariance would simply become (approximately, disregarding the $\varepsilon_{i}$ correction, which simply changes weights) the cross sectional variance of $\mathbb{E}_{i}[D]$, which is always strictly positive, as long as investors disagree. Fundamental trading can make some optimists sellers and some pessimists buyers, but in order to overturn [OBPS], a particular pattern of fundamental trading is required. In other words, a priori, disagreement trading creates a force that suggests [OBPS] holds, but fundamental trading does not create any prediction about whether [OBPS] holds or not.

\section{G Linear combination between planner's belief and investors' beliefs}

Throughout the paper, I assume that the planner maximizes welfare using a single distribution of payoffs for all investors - in principle, the true distribution. It is straightforward to generalize the results to a planner that puts weight $\alpha$ on his own belief and weight $1-\alpha$ on the belief of each investor. In that case, the new optimal tax $\tau_{\alpha}^{*}$ looks turns out to be a linear combination of both taxes. In the baseline model, because the optimal tax for a planner that respects agents beliefs is $\tau^{*}=0$, the optimal tax becomes:

$$
\tau_{\alpha}^{*}=\alpha \tau^{*}
$$

Where $\tau^{*}$ is given by equation (13). The same logic applies to other extensions of the baseline model. The case with $\alpha=1$ is the leading case analyzed in the paper. The case with $\alpha=0$ is the one used in subsections 3.4 and 6.3 .

\section{H Dynamic model with production}

I briefly analyze here an extension of the dynamic model to include the possibility of Walrasian production. This combines the result of sections 6 and 7. Market clearing at each state is given by $\int \Delta X_{t i} d F(i)=Q_{t}+S_{t}$, where I assume a law of motion for the number of trees $Q_{t+1}=Q_{t}+S_{t}$ where we could allow $S_{t}$ (investment) to be negative (let's assume that $S_{t} \geq-Q_{t}$ ). Allowing for positive depreciation is straightforward. Assume also that $\beta=1$ and $R=1$.

Under the welfare criterion used in this paper, we can write an approximated optimal tax around the case in which all investors and producers have constant marginal utility in the following way:

$$
\tau^{*}=\frac{\mathbb{E}\left[\sum_{t=1}^{T} \int\left(\mathbb{E}_{t}\left[D_{t+1}+P_{t+1}\right]-\mathbb{E}_{t i}\left[D_{t+1}+P_{t+1}\right]\right) \frac{d X_{t i}}{d \tau} d F(i)\right]}{\mathbb{E}\left[\sum_{t=1}^{T} \int P_{t} \int \operatorname{sgn}\left(\Delta X_{t i}\right)\left(1-\kappa_{t i}\right) \frac{d X_{t i}}{d \tau} d F(i)\right]}
$$


The term corresponding to each node in the numerator of this expression can be written as:

$$
\int\left(\mathbb{E}_{t}\left[D_{t+1}+P_{t+1}\right]-\mathbb{E}_{t i}\left[D_{t+1}+P_{t+1}\right]\right) \frac{d X_{t i}}{d \tau} d F(i)=\zeta_{t}(\tau) \mathbb{E}_{F, \mathcal{T}}\left[\left(\mathbb{E}_{t}\left[D_{t+1}+P_{t+1}\right]-\mathbb{E}_{t i}\left[D_{t+1}+P_{t+1}\right]\right) \frac{d X_{t i}}{d \tau}\right]
$$

where we can write:

$$
\begin{gathered}
\mathbb{E}_{F, \mathcal{T}}\left[\left(\mathbb{E}_{t}\left[D_{t+1}+P_{t+1}\right]-\mathbb{E}_{t i}\left[D_{t+1}+P_{t+1}\right]\right) \frac{d X_{t i}}{d \tau}\right]= \\
=\operatorname{Cov}_{F, \mathcal{T}}\left[\left(\mathbb{E}_{t}\left[D_{t+1}+P_{t+1}\right]-\mathbb{E}_{t i}\left[D_{t+1}+P_{t+1}\right]\right) \frac{d X_{t i}}{d \tau}\right]+\mathbb{E}_{F, \mathcal{T}}\left[\mathbb{E}_{t}\left[D_{t+1}+P_{t+1}\right]-\mathbb{E}_{t i}\left[D_{t+1}+P_{t+1}\right]\right] \mathbb{E}_{F, \mathcal{T}}\left[\frac{d X_{t i}}{d \tau}\right] \\
=-\operatorname{Cov}_{F, \mathcal{T}}\left[\mathbb{E}_{t i}\left[D_{t+1}+P_{t+1}\right], \frac{d X_{t i}}{d \tau}\right]+\left(\mathbb{E}_{t}\left[D_{t+1}+P_{t+1}\right]-\mathbb{E}_{F, \mathcal{T}}\left[\mathbb{E}_{t i}\left[D_{t+1}+P_{t+1}\right]\right]\right) \frac{d S_{t}}{d \tau}
\end{gathered}
$$

where $\mathbb{E}_{F, \mathcal{T}, t}$ denotes the cross sectional expectation of active investor at a given node in period $t$. Where the last line uses the properties of market clearing. This expression is identical to the one discussed in subsection 7.1.

I now show how in a dynamic context the planner only needs to know an expectation/covariance of the terms in the numerator:

$$
\begin{gathered}
\sum_{t=1}^{T} \mathbb{E}\left[\mathbb{E}_{F, \mathcal{T}}\left[\left(\mathbb{E}_{t}\left[D_{t+1}+P_{t+1}\right]-\mathbb{E}_{t i}\left[D_{t+1}+P_{t+1}\right]\right) \frac{d X_{t i}}{d \tau}\right]\right]= \\
=\sum_{t=1}^{T}\left(\mathbb{E}\left[-\operatorname{Cov}_{F, \mathcal{T}}\left[\mathbb{E}_{t i}\left[D_{t+1}+P_{t+1}\right], \frac{d X_{t i}}{d \tau}\right]\right]-\mathbb{E}\left[\mathbb{E}_{t}\left[D_{t+1}+P_{t+1}\right]-\mathbb{E}_{F, \mathcal{T}}\left[\mathbb{E}_{t i}\left[D_{t+1}+P_{t+1}\right]\right] \frac{d S_{t}}{d \tau}\right]\right)
\end{gathered}
$$

Note that:

$$
\begin{gathered}
\mathbb{E}\left[\mathbb{E}_{t}\left[D_{t+1}+P_{t+1}\right]-\mathbb{E}_{F, \mathcal{T}}\left[\mathbb{E}_{t i}\left[D_{t+1}+P_{t+1}\right]\right] \frac{d S_{t}}{d \tau}\right]= \\
=\mathbb{E}\left[\mathbb{E}_{t}\left[D_{t+1}+P_{t+1}\right]-\mathbb{E}_{F, \mathcal{T}}\left[\mathbb{E}_{t i}\left[D_{t+1}+P_{t+1}\right]\right]\right] \mathbb{E}\left[\frac{d S_{t}}{d \tau}\right]-\operatorname{Cov}\left[\mathbb{E}_{t}\left[D_{t+1}+P_{t+1}\right]-\mathbb{E}_{F, \mathcal{T}}\left[\mathbb{E}_{t i}\left[D_{t+1}+P_{t+1}\right]\right], \frac{d S_{t}}{d \tau}\right]
\end{gathered}
$$

So the numerator of the optimal tax is given by the period-by-period sum of three terms. First, the expected dispersion across states. Second, average mistake across states of nature times the average investment sensitivity. Third, the covariance between the sign of the aggregate distortions and the investment derivative across states.

For instance, assuming that investors are correct on average across states, but not state-by-state, that is, $\mathbb{E}\left[\mathbb{E}_{F}\left[\mathbb{E}_{t}\left[D_{t+1}+P_{t+1}\right]-\mathbb{E}_{t i}\left[D_{t+1}+P_{t+1}\right]\right]\right]=0$, we can write the optimal tax as:

$$
\tau^{*}=\frac{\sum_{t=1}^{T} \mathbb{E}\left[-\operatorname{Cov}_{F, \mathcal{T}}\left[\mathbb{E}_{t i}\left[D_{t+1}+P_{t+1}\right], \frac{d X_{t i}}{d \tau}\right]\right]+\operatorname{Cov}\left[\mathbb{E}_{t}\left[D_{t+1}+P_{t+1}\right]-\mathbb{E}_{F, \mathcal{T}}\left[\mathbb{E}_{t i}\left[D_{t+1}+P_{t+1}\right]\right], \frac{d S_{t}}{d \tau}\right]}{\mathbb{E}\left[\sum_{t=1}^{T} \int P_{t} \int \operatorname{sgn}\left(\Delta X_{t i}\right)\left(1-\kappa_{t i}\right) \frac{d X_{t i}}{d \tau} d F(i)\right]}
$$

The first term in the denominator is the same one as in an exchange economy. The new term is the second one. This is a covariance - again, not a variance - over time of the marginal effect of a tax change in investment with the average belief distortion. It is simply an average over time of the effect discussed in section 7. Intuitively, if the (constant) transaction tax is an automatic stabilizer of investment it can be welfare improving.

A similar logic to the one discussed in subsection 6.3 also applies in this context, after taking into account the welfare of the producer. The optimal tax is set to provide the maximum level of insurance to investors and the producer. These results are available under request.

\section{General model: additional results}

The following two results complement the analysis of section 6 . 


\section{General static model}

The counterpart of the baseline for a general utility specification is the following. When $T=2$ and consumption at $t=1$ is fixed, the optimal tax becomes:

$$
\tau^{*}=\frac{\int \lambda_{i} \operatorname{Cov}\left(Y_{i}, U_{i}^{\prime}\left(C_{2 i}\right)\left(\frac{D}{P_{1}}-R\right)\right) \frac{d X_{1 i}}{d \tau} d F(i)}{\int \operatorname{sgn}\left(\Delta X_{1 i}\right) \frac{d X_{1 i}}{d \tau} d F(i)}
$$

When $U_{i}^{\prime}(\cdot) \approx 1$ and [NR] holds, the optimal tax becomes identical to the CARA-Normal case, introduced in proposition 2. This result shares the logic of the classic Arrow-Pratt approximation.

The sign and magnitude of the optimal tax is intuitive. The value of $\operatorname{Cov}\left[Y_{i}, U_{i}^{\prime}\left(C_{2 i}\right)\left(\frac{D}{P}-R\right)\right]$ is positive when investors are optimistic $-Y_{i}$ is high when $D$ is high - and it is negative when investors are pessimistic. As long as optimists have $\frac{d X_{1 i}}{d \tau}<0$ and pessimists have $\frac{d X_{1 i}}{d \tau}>0$, this would call for a positive tax.

\section{Labor supply/additional choice variables}

The results of the paper do not change when investors' incomes are endogenously generated. For instance, assume that investors decide to work $N_{t i}$ hours at a wage $W_{t i}$, maximizing flow utility given by $U_{i}\left(C_{t i}, N_{t i}\right)$. In that case, the optimal tax formula remains unchanged after substituting $U_{i}^{\prime}\left(C_{t i}\right)$ with $\frac{\partial U_{i}\left(C_{t i}, N_{t i}\right)}{\partial C_{t i}}$. If consumption and labor are separable, all the previous results apply directly. This logic, which is identical to the one used to characterize standard Euler equations, applies to any other static choice variable. 


\section{References}

Aiyagari, S Rao, Albert Marcet, Thomas J Sargent, and Juha Seppala. 2002. “Optimal Taxation without State-Contingent Debt." Journal of Political Economy, 110(6): 1220-1254.

Akerlof, G.A., and J.L. Yellen. 1985. “A near-rational model of the business cycle, with wage and price inertia." The Quarterly Journal of Economics, 100(Supplement): 823-838.

Albagli, Elias, Christian Hellwig, and Aleh Tsyvinski. 2011. “Information Aggregation, Investment, and Managerial Incentives." National Bureau of Economic Research.

Amihud, Yakov, Haim Mendelson, and Lasse Heje Pedersen. 2005. "Liquidity and Asset Prices." Foundations and Trends in Finance, 1(4): 269-364.

Amihud, Y., and H. Mendelson. 1986. "Asset pricing and the bid-ask spread." Journal of financial Economics, 17(2): 223-249.

Amromin, Gene, and Steven Sharpe. 2012. "From the horse's mouth: How do investor expectations of risk and return vary with economic conditions?"

Angeletos, George-Marios, Guido Lorenzoni, and Alessandro Pavan. 2010. "Beauty contests and irrational exuberance: A neoclassical approach." National Bureau of Economic Research.

Arrow, Kenneth J. 1971. "The theory of risk aversion." Essays in the theory of risk-bearing, 90-120.

Auerbach, Alan J, and James R Hines Jr. 2002. “Taxation and economic efficiency." Handbook of public economics, 3: $1347-1421$.

Aumann, Robert J. 1976. “Agreeing to disagree." The annals of statistics, 4(6): 1236-1239.

Banerjee, S. 2011. "Learning from Prices and the Dispersion in Beliefs." Review of Financial Studies, 24(9): 3025-3068.

Barber, Brad, and Terrance Odean. 2013. "The behavior of individual investors." Handbook of the Economics of Finance, Vol.2.

Benartzi, Shlomo. 2001. "Excessive extrapolation and the allocation of 401 (k) accounts to company stock." The Journal of Finance, 56(5): 1747-1764.

Ben-David, Itzhak, John R Graham, and Campbell R Harvey. 2013. "Managerial Miscalibration." Quarterly Journal of Economics, forthcoming.

Bernheim, B Douglas. 2009. “Behavioral welfare economics.” Journal of the European Economic Association, 7(2-3): 267-319.

Bernheim, B Douglas, and Antonio Rangel. 2009. "Beyond revealed preference: choice-theoretic foundations for behavioral welfare economics." The Quarterly Journal of Economics, 124(1): 51-104.

Bianchi, Javier. 2011. "Overborrowing and Systemic Externalities in the Business Cycle." The American Economic Review, 101(7): 3400-3426.

Black, Fischer. 1986. “Noise." The Journal of Finance, 41(3): 529-543.

Blume, Lawrence, and David Easley. 2010. "Heterogeneity, selection, and wealth dynamics." Annu. Rev. Econ., 2(1): 425-450.

Blume, Lawrence E, Timothy Cogley, David A Easley, Thomas J Sargent, and Viktor Tsyrennikov. 2013. “Welfare, Paternalism and Market Incompleteness." Working Paper.

Brunnermeier, M., A. Simsek, and W. Xiong. 2012. “A Welfare Criterion for Models with Distorted Beliefs.” Working Paper.

Brunnermeier, M.K. 2001. Asset pricing under asymmetric information: Bubbles, crashes, technical analysis, and herding. OUP Oxford.

Budish, Eric, Peter Cramton, and John Shim. 2013. “The High-Frequency Trading Arms Race: Frequent Batch Auctions as a Market Design Response." Working Paper.

Buss, Adrian, and Bernard Dumas. 2013. "Financial-Market Equilibrium with Friction." NBER Working Paper.

Campbell, J.Y. 2006. “Household finance.” The Journal of Finance, 61(4): 1553-1604.

Campbell, J.Y., and K.A. Froot. 1994. “International experiences with securities transaction taxes." In The internationalization of equity markets. 277-308. University of Chicago Press. 
Chari, VV, and Patrick J. Kehoe. 1999. “Optimal Fiscal and Monetary Policy. In Handbook of Macroeconomics, edited by John B. Taylor and Michael Woodford, Vol. 1C. Elsevier."

Chetty, Raj. 2009. "Sufficient Statistics for Welfare Analysis: A Bridge Between Structural and Reduced-Form Methods." Annual Review of Economics, 1(1): 451-488.

Cochrane, John H. 2005. Asset Pricing: (Revised). . Revised ed., Princeton University Press.

Cochrane, John H. 2013. “Finance: Function Matters, Not Size.” The Journal of Economic Perspectives, 27(2): 29-50.

Constantinides, G.M. 1986. “Capital market equilibrium with transaction costs.” The Journal of Political Economy, $842-862$.

Dang, Tri V., Gary Gorton, and Bengt Holmstrom. 2009. “Opacity and the optimality of debt for liquidity provision." MIT Working paper.

Davila, Eduardo. 2011. “Dissecting Fire Sales Externalities.” Harvard Working Paper.

De Long, J.B., A. Shleifer, L.H. Summers, and R.J. Waldmann. 1990. “Noise Trader Risk in Financial Markets." The Journal of Political Economy, 98(4): 703-738.

Diamond, D.W., and R.E. Verrecchia. 1981. "Information aggregation in a noisy rational expectations economy." Journal of Financial Economics, 9(3): 221-235.

Diamond, Peter A. 1973. "Consumption externalities and imperfect corrective pricing." The Bell Journal of Economics and Management Science, 526-538.

Døskeland, Trond M, and Hans K Hvide. 2011. “Do individual investors have asymmetric information based on work experience?" The Journal of Finance, 66(3): 1011-1041.

Dow, James, and Gary Gorton. 1997. “Stock market efficiency and economic efficiency: is there a connection?" The Journal of Finance, 52(3): 1087-1129.

Dow, J., and R. Rahi. 2000. “Should Speculators Be Taxed?” The journal of business, 73(1): 89-107.

Duffie, Darrell. 2001. Dynamic Asset Pricing Theory, Third Edition. Princeton University Press.

Duffie, D., N. Gârleanu, and L.H. Pedersen. 2005. “Over-the-Counter Markets.” Econometrica, 73(6): $1815-1847$.

Fama, Eugene F. 1976. Foundations of finance: portfolio decisions and securities prices. Basic Books New York, NY.

Fama, Eugene F, and Kenneth R French. 2007. "Disagreement, tastes, and asset prices." Journal of Financial Economics, 83(3): 667-689.

Friedman, Milton. 1953. Essays in positive economics. Vol. 231, University of Chicago Press.

Fudenberg, Drew, and Jean Tirole. 1991. Game Theory. MIT Press.

Gârleanu, Nicolae, and Lasse Heje Pedersen. 2012. “Dynamic Trading with Predictable Returns and Transaction Costs.”

Gârleanu, Nicolae, Stavros Panageas, and Jianfeng Yu. 2013. "Financial Entanglement: A Theory of Incomplete Integration, Leverage, Crashes, and Contagion." Working Paper.

Geanakoplos, J. 2010. “The leverage cycle.” NBER Macroeconomics Annual, 24(1): 1-66.

Geanakoplos, John, and Herakles Polemarchakis. 1986. "Existence, regularity, and constrained suboptimality of competitive allocations when the asset market is incomplete." Essays in honor of Kenneth J. Arrow, 3: 65-95.

Gilboa, Itzhak, Larry Samuelson, and David Schmeidler. 2012. “No-Betting Pareto Dominance.”

Glosten, L.R., and P.R. Milgrom. 1985. “Bid, ask and transaction prices in a specialist market with heterogeneously informed traders." Journal of financial economics, 14(1): 71-100.

Goldstein, Itay, and Alexander Guembel. 2008. "Manipulation and the allocational role of prices." The Review of Economic Studies, 75(1): 133-164.

Goldstein, Itay, Emre Ozdenoren, and Kathy Yuan. 2013. "Trading frenzies and their impact on real investment." Journal of Financial Economics. 
Gollier, Christian. 2001. The economics of risk and time. The MIT Press.

Goulder, Lawrence H. 1995. “Environmental taxation and the double dividend: a reader's guide." International Tax and Public Finance, 2(2): 157-183.

Greenwood, Robin, and Andrei Shleifer. 2013. "Expectations of Returns and Expected Returns." NBER Working Paper, , (w18686).

Gromb, Denis, and Dimitri Vayanos. 2002. "Equilibrium and welfare in markets with financially constrained arbitrageurs." Journal of Financial Economics, 66(2-3): 361-407.

Grossman, S.J., and J.E. Stiglitz. 1980. “On the impossibility of informationally efficient markets." The American Economic Review, 393-408.

Guasoni, P., and J. Muhle-Karbe. 2012. “Portfolio Choice with Transaction Costs: a User's Guide.” Working Paper.

Habermeier, K., and A.A. Kirilenko. 2003. “Securities Transaction Taxes and Financial Markets." IMF Staff Papers, 165-180.

Haltiwanger, John, and Michael Waldman. 1985. "Rational expectations and the limits of rationality: An analysis of heterogeneity." The American Economic Review, 75(3): 326-340.

Haltiwanger, John, and Michael Waldman. 1989. "Limited rationality and strategic complements: the implications for macroeconomics." The Quarterly Journal of Economics, 104(3): 463-483.

Harberger, Arnold C. 1964. “The measurement of waste.” The American Economic Review, 54(3): 58-76.

Harrison, J.M., and D.M. Kreps. 1978. "Speculative investor behavior in a stock market with heterogeneous expectations." The Quarterly Journal of Economics, 92(2): 323-336.

Hart, Oliver D. 1974. “On the existence of equilibrium in a securities model.” Journal of economic theory, 9(3): $293-311$.

Hart, Oliver D. 1975. “On the optimality of equilibrium when the market structure is incomplete." Journal of Economic Theory, 11(3): 418-443.

Hayashi, F. 1982. “Tobin's marginal q and average q: A neoclassical interpretation." Econometrica: Journal of the Econometric Society, 213-224.

Hayek, FA. 1945. "The Use of Knowledge in Society." The American Economic Review, 35(4): 519-530.

Hellwig, M.F. 1980. “On the aggregation of information in competitive markets.” Journal of Economic Theory, 22(3): 477-498.

Hendren, Nathaniel. 2013. "The Policy Elasticity." Harvard Working Paper.

He, Zhiguo, and Péter Kondor. 2012. "Inefficient Investment Waves." National Bureau of Economic Research.

Hong, H., and J.C. Stein. 2007. "Disagreement and the stock market.” The Journal of Economic Perspectives, 21(2): 109-128.

Hong, Harrison, and Jeremy C Stein. 1999. "A unified theory of underreaction, momentum trading, and overreaction in asset markets." The Journal of Finance, 54(6): 2143-2184.

Huang, Chi-fu, and Robert H Litzenberger. 1988. Foundations for financial economics. Vol. 4, North-Holland New York.

Ingersoll, J.E. 1987. Theory of financial decision making. Vol. 3, Rowman \& Littlefield Pub Incorporated.

Jarrow, Robert. 1980. “Heterogeneous expectations, restrictions on short sales, and equilibrium asset prices." The Journal of Finance, 35(5): 1105-1113.

Jeanne, Oliver, and Anton Korinek. 2010. “Managing credit booms and busts: A Pigouvian taxation approach.” Working paper.

Jones, C.M., and P.J. Seguin. 1997. "Transaction costs and price volatility: evidence from commission deregulation." The American Economic Review, 728-737.

Kopczuk, Wojciech. 2003. “A note on optimal taxation in the presence of externalities." Economics Letters, 80(1): 81-86.

Korinek, Anton. 2009. "Systemic risk-taking: amplification effects, externalities and regulatory responses." Working Paper, Maryland.

Kreps, David M. 2012. Microeconomic Foundations I: Choice and Competitive Markets. Princeton University Press. 
Leland, H.E. 1992. “Insider trading: Should it be prohibited?" Journal of Political Economy, 859-887.

Lintner, John. 1969. “The aggregation of investors' diverse judgments and preferences in purely competitive security markets." Journal of Financial and Quantitative Analysis, 4(4): 347-400.

Liu, H. 2005. "Optimal consumption and investment with transaction costs and multiple risky assets." The Journal of Finance, 59(1): 289-338.

Lo, A.W., H. Mamaysky, and J. Wang. 2004. "Asset Prices and Trading Volume under Fixed Transactions Costs." Journal of Political Economy, 112(5).

Lorenzoni, Guido. 2008. “Inefficient credit booms.” Review of Economic Studies, 75(3): 809-833.

Lucas, Robert E. 1987. Models of business cycles. Basil Blackwell New York.

Lucas, Robert E. 1990. “Liquidity and interest rates.” Journal of Economic Theory, 50(2): 237-264.

Lucas, Robert E, and Nancy L Stokey. 1983. “Optimal fiscal and monetary policy in an economy without capital." Journal of monetary Economics, 12(1): 55-93.

Magill, Michael, and Martine Quinzii. 2002. “Theory of incomplete markets, volume 1." MIT Press Books, 1.

Massa, Massimo, and Andrei Simonov. 2006. "Hedging, familiarity and portfolio choice." Review of Financial Studies, 19(2): 633-685.

McCulloch, Neil, and Grazia Pacillo. 2011. “The Tobin Tax - A Review of the Evidence.” Working Paper, Institute of Development Studies.

Miller, E.M. 1977. “Risk, uncertainty, and divergence of opinion.” The Journal of Finance, 32(4): 1151-1168.

Morris, Stephen. 1995. "The common prior assumption in economic theory." Economics and philosophy, 11: 227-227.

Mullainathan, Sendhil, Joshua Schwartzstein, and William Congdon. 2012. "A Reduced-Form Approach to Behavioral Public Finance." Annual Review of Economics, 4: 511-540.

O’Donoghue, Ted, and Matthew Rabin. 2006. “Optimal sin taxes." Journal of Public Economics, 90(10): 1825-1849.

Ozdenoren, Emre, and Kathy Yuan. 2008. “Feedback effects and asset prices.” The journal of finance, 63(4): 1939-1975.

Posner, Eric, and Glen Weyl. 2013. “Benefit-Cost Analysis for Financial Regulation.” American Economic Review, 103(3).

Pratt, John W. 1964. "Risk aversion in the small and in the large." Econometrica: Journal of the Econometric Society, $122-136$.

Roll, R. 1989. "Price volatility, international market links, and their implications for regulatory policies." Journal of Financial Services Research, 3(2): 211-246.

Ross, S.A. 1989. "Commentary: using tax policy to curb speculative short-term trading." Journal of Financial Services Research, 3: 117-120.

Sandmo, Agnar. 1975. “Optimal taxation in the presence of externalities." The Swedish Journal of Economics, 86-98.

Sandmo, Agnar. 1985. "The effects of taxation on savings and risk taking." Handbook of Public Economics, 1: $265-311$.

Sandroni, Alvaro, and Francesco Squintani. 2007. "Overconfidence, insurance, and paternalism." The American Economic Review, 97(5): 1994-2004.

Scheinkman, Jose A, and Wei Xiong. 2003. "Overconfidence and Speculative Bubbles." Journal of Political Economy, 111(6).

Schwert, G William, and Paul J Seguin. 1993. "Securities transaction taxes: an overview of costs, benefits and unresolved questions." Financial Analysts Journal, 27-35.

Shleifer, Andrei. 2000. Inefficient Markets: An Introduction to Behavioral Finance (Clarendon Lectures in Economics). Oxford University Press, USA.

Simsek, Alp. 2013a. “Belief disagreements and collateral constraints." Econometrica, 81(1): 1-53.

Simsek, Alp. 2013b. "Speculation and Risk Sharing with New Financial Assets." The Quarterly Journal of Economics. 
Spinnewijn, Johannes. 2012. "Unemployed but optimistic: Optimal insurance design with biased beliefs." Working Paper.

Stein, Jeremy C. 2012. "Monetary policy as financial stability regulation." The Quarterly Journal of Economics, 127(1): 57-95.

Stiglitz, J.E. 1989. “Using tax policy to curb speculative short-term trading.” Journal of financial services research, 3(2): 101-115.

Subrahmanyam, A. 1998. “Transaction Taxes and Financial Market Equilibrium.” The Journal of Business, 71(1): 81-118.

Subrahmanyam, Avanidhar, and Sheridan Titman. 1999. “The going-public decision and the development of financial markets." The Journal of Finance, 54(3): 1045-1082.

Summers, L.H., and V.P. Summers. 1989. “When financial markets work too well: a cautious case for a securities transactions tax." Journal of financial services research, 3(2): 261-286.

Tobin, J. 1969. "A general equilibrium approach to monetary theory." Journal of money, credit and banking, 1(1): 15-29.

Tobin, J. 1978. “A proposal for international monetary reform.” Eastern Economic Journal, 4(3/4): 153-159.

ul Haq, M., I. Kaul, and I. Grunberg. 1996. The Tobin tax: coping with financial volatility. Oxford University Press, USA.

Umlauf, S.R. 1993. “Transaction taxes and the behavior of the Swedish stock market." Journal of Financial Economics, 33(2): 227240.

Vayanos, D. 1998. “Transaction costs and asset prices: A dynamic equilibrium model." Review of financial studies, 11(1): 1-58.

Vayanos, Dimitri, and Jiang Wang. 2012. “Market Liquidity - Theory and Empirical Evidence." Foundations and Trends Journal Articles, 6(4): 221-317.

Xiong, W. 2012. “Bubbles, Crises, and Heterogeneous Beliefs." Handbook for Systemic Risk. 